\title{
Left atrial vascular and metabolic remodeling in pig models of atrial fibrillation
}

Citation for published version (APA):

Nasrallah, H. M. (2015). Left atrial vascular and metabolic remodeling in pig models of atrial fibrillation. [Doctoral Thesis, Maastricht University]. Uitgeverij BOXPress. https://doi.org/10.26481/dis.20151216hn

Document status and date:

Published: 01/01/2015

DOI:

10.26481/dis.20151216hn

Document Version:

Publisher's PDF, also known as Version of record

\section{Please check the document version of this publication:}

- A submitted manuscript is the version of the article upon submission and before peer-review. There can be important differences between the submitted version and the official published version of record.

People interested in the research are advised to contact the author for the final version of the publication, or visit the DOI to the publisher's website.

- The final author version and the galley proof are versions of the publication after peer review.

- The final published version features the final layout of the paper including the volume, issue and page numbers.

Link to publication

\footnotetext{
General rights rights.

- You may freely distribute the URL identifying the publication in the public portal. please follow below link for the End User Agreement:

www.umlib.nl/taverne-license

Take down policy

If you believe that this document breaches copyright please contact us at:

repository@maastrichtuniversity.nl

providing details and we will investigate your claim.
}

Copyright and moral rights for the publications made accessible in the public portal are retained by the authors and/or other copyright owners and it is a condition of accessing publications that users recognise and abide by the legal requirements associated with these

- Users may download and print one copy of any publication from the public portal for the purpose of private study or research.

- You may not further distribute the material or use it for any profit-making activity or commercial gain

If the publication is distributed under the terms of Article $25 \mathrm{fa}$ of the Dutch Copyright Act, indicated by the "Taverne" license above, 


\section{Left atrial vascular and metabolic remodeling in pig models of atrial fibrillation}


Copyright 2015 @ Hussein M. Nasrallah

ISBN: $978-94-6295-232-4$

Cover by Hussein M. Nasrallah

Lay-out by Proefschriftmaken.nl || Uitgeverij BOXPress

Printed by Proefschriftmaken.nl || Uitgeverij BOXPress

Published by Uitgeverij BOXPress, 's-Hertogenbosch

All rights reserved. No part of this book may be reproduced, stored in a database or retrieval system, or published in any form or by any means, without prior permissi-on in writing from the author. 


\title{
Left atrial vascular and metabolic remodeling in pig models of atrial fibrillation
}

\author{
PROEFSCHRIFT
}

Ter verkrijging de graad van doctor aan de Universiteit Maastricht, op gezag van de Rector Magnificus, Prof. Dr. L.L.G. Soete, volgens het besluit van het College van Decanen in het openbaar te verdedigen op

Woensdag 16 December 2015 om 10.00 uur

door

Hussein M. Nasrallah 


\section{PROMOTOR}

Prof. dr. U. Schotten

\section{COPROMOTOR}

Dr. S. Verheule

\section{BEOORDELINGSCOMMISSIE}

Prof. dr. H.J.G.M. Crijns (voorzitter)

Prof. dr. M.J. Post

Prof. dr. D.J.G.M. Duncker, Erasmus MC Rotterdam

Prof. dr. E. van Bavel, AMC Amsterdam

This research is supported by CARIM (Cardiovascular Research Institute Maastricht) the European network for translational research in atrial fibrillation (EUTRAF) and Leducq Foundation (Project ENAFRA)
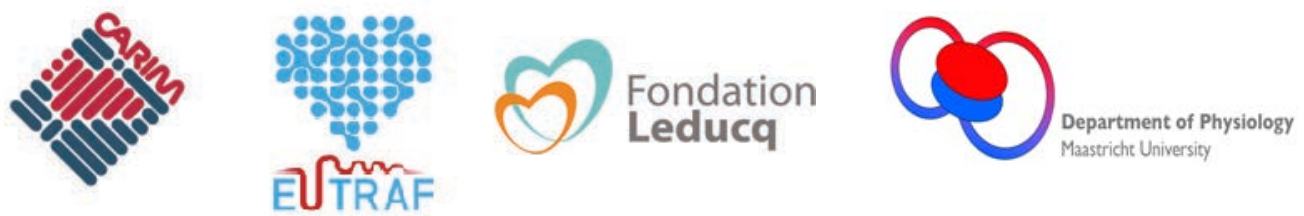
In the Name of God the most compassionate the most merciful بسم الله الرحمن الرحيم 



\section{TABLE OF CONTENTS}

CHAPTER 1 General Introduction 9

CHAPTER 2 Left atrial coronary oxygen supply in a pig model 17 of congestive heart failure: vascular remodeling and reduced oxygen efficiency

CHAPTER 3 Atrial fibrillation causes remodeling of vascular structure 39

CHAPTER 4 Atrial fibrillation alters the behavior of atrial and 57 ventricular small muscular arteries

CHAPTER 5 Early metabolic and mitochondrial remodeling in a 75 pig model of atrial fibrillation

$\begin{array}{lll}\text { CHAPTER } 6 \text { General Discussion } & 105\end{array}$

$\begin{array}{lll}\text { APPENDIX Summary } & 118\end{array}$

122

Valorization 124

$\begin{array}{ll}\text { About the author } & 128\end{array}$

$\begin{array}{ll}\text { List of publications } & 129\end{array}$

Acknowledgment 130 



\section{General Introduction}

Hussein M. Nasrallah

1

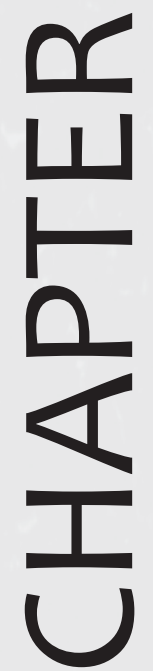




\section{GENERAL INTRODUCTION}

Atrial fibrillation (AF) is the most common sustained tachyarrhythmia in clinical practice, affecting approximately $1 \%$ of the general population. AF is characterized by fast and irregular atrial activation. Patients with AF have a significantly higher risk of thromboembolic events, including strokes ${ }^{1}$. In addition, AF is associated with lower quality of life because it diminishes cardiac performance ${ }^{2}$. The current cost associated with AF is a tremendous financial burden on the healthcare system ${ }^{3}$. The prevalence of AF increases with age (10\% of people aged $>75$ suffer from AF) ${ }^{4,5}$. Because of the ageing of the population in the Western world, the number of AF patients and associated health care costs are expected to increase in the coming decades.

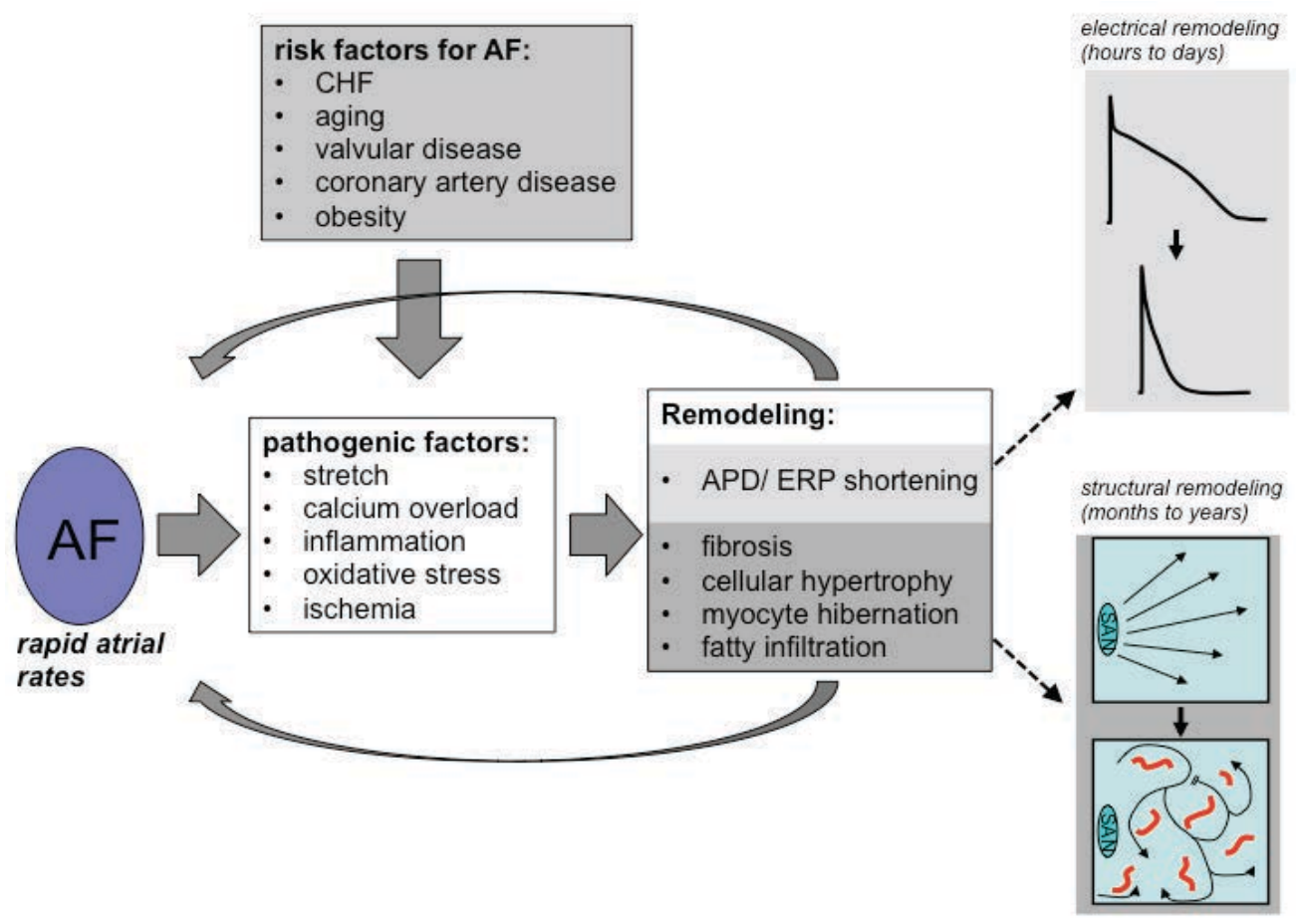

Figure 1. Mechanisms involved in atrial remodeling

\section{PROGRESSION OF AF}

Both in AF patients and in animal models, the stability of AF gradually increases. This 'entrenchment' of AF in patients is evidenced by a gradual prolongation of the duration of AF episodes, ultimately leading to persistent AF (non-self terminating, but still amenable to pharmacological cardioversion) and permanent AF (non-self terminating and insensitive to pharmacological cardioversion). 
Two major mechanisms that are thought to be responsible for perpetuation of AF are electrical and structural remodeling (figure 1). Electrical remodeling is a relatively fast process that occurs within hours to days and involves shortening of the action potential duration and effective refractory period (ERP) (figure 2$)^{6}$. As a result, tissue can be re-excited more rapidly, allowing higher frequencies of circulating waves and/ or fibrillation waves to coexist. However, the contribution of electrical remodeling to AF stabilization is probably modest. In goats, 24 hours of AF leads to maximal reduction in atrial ERP, whereas stability of AF is still low, having increased from seconds in control animal to minutes. AF stability gradually increases over the ensuing weeks to months ${ }^{7}$, suggesting the importance of additional remodeling processes. Structural remodeling is a slow remodeling process that becomes manifest at later stages of the pathogenesis of AF (figure 2). The structural abnormalities occurring in atrial myocardium as a result of AF, in particular endomysial fibrosis and myocyte hypertrophy, result in the loss of transverse connections between myocyte bundles, thereby increasing the complexity of fibrillatory conduction and the stability of $\mathrm{AF}^{8}$, 9 .Whereas electrical remodeling can be viewed as an adaptation to calcium overload ${ }^{10}$ the mechanism(s) responsible for structural remodeling are not well understood.

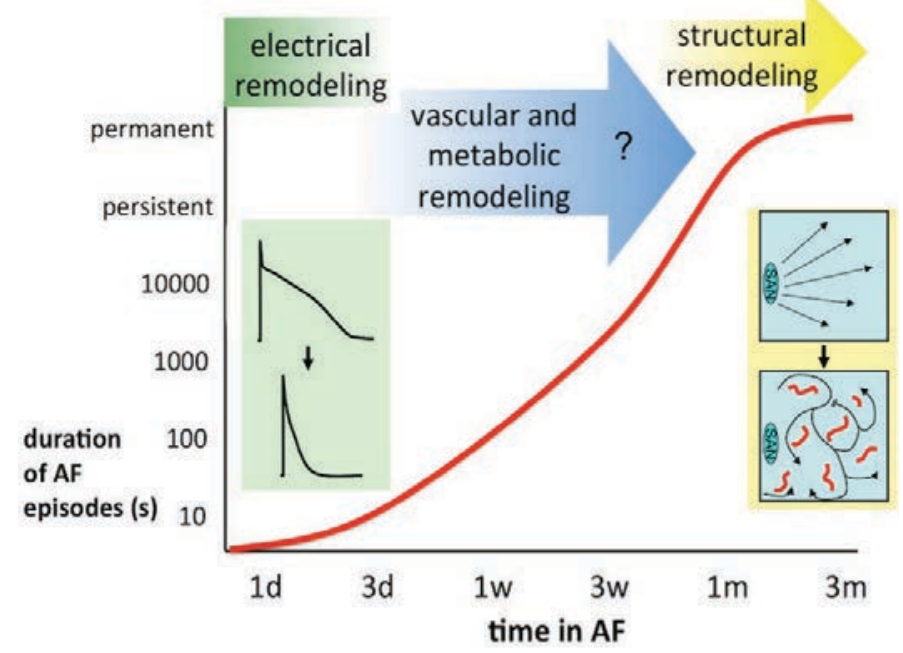

Figure 2. AF remodeling processes involved in AF stabilization. The existence and importance of electrical and structural remodeling is generally accepted. The putative process of vascular and metabolic remodeling may take place during the most important phase of AF stabilization, may directly affect myocyte electrophysiology, and may be a causative mechanism in the development of structural remodeling. 


\section{AF AND ENERGY DEPLETION}

We recently showed that during acute AF in healthy pigs, a major part of the reserve in coronary blood flow and oxygen extraction is recruited. Nevertheless, atrial lactate production increased, indicating occurrence of a supply demand mismatch ${ }^{11}$. In addition, a hyperemic phase follows a short episode of AF, indicating that an oxygen debt had been incurred ${ }^{12}$. Other studies support the occurrence of (supply/ demand) ischemia during prolonged AF. Expression of the hypoxia marker HIF1a is increased in AF patients and in goats in the first weeks of AF ${ }^{13}{ }^{14}$. In goats, AF also lead to a transient reduction in phosphocreatine within the first month ${ }^{15}$, and the phenotype of dedifferentiation/ hibernation of atrial myocytes is reminiscent of that of ventricular myocytes during low-flow ischemia ${ }^{16}$. Therefore, it is conceivable that the fast and irregular activation in the atria of AF subjects may lead to a state of supply/demand ischemia that may be an important contributor to the creation of a substrate for AF.

\section{OUTLINE OF THIS THESIS}

The first objective of this thesis was to determine whether atrial coronary flow regulation is affected in a large animal model of AF due to underlying structural heart disease. The second aim was to investigate whether AF leads to vascular and metabolic remodeling.

Clinically, congestive heart failure (CHF) is a strong independent risk factor for $A F{ }^{17}$. Approximately $50 \%$ of patients with severe heart failure (NYHA class III/IV) develop AF. In these patients, the occurrence of AF can lead to further exacerbation of heart failure ${ }^{18}$. In a dog model of CHF, pronounced (replacement) fibrosis has been observed, which is thought to explain the increased AF stability in this model ${ }^{19}$. We originally hypothesized that CHF leads to atrial vascular remodeling that would increase the atrial susceptibility to supply/ demand ischemia during circumstances such increased heart rate of AF. To this end, we investigated atrial coronary blood flow regulation and vascular structure in a pig model of CHF (chapter 2).

To investigate remodeling of vascular structure as a result of AF itself, we studied two relatively early time points in the pathogenesis of AF in a pig model with rapid atrial pacing (RAP). At 1 and 5 weeks of RAP, we studied the expression of markers of hypoxia (HIF1a) and angiogenesis (VEGF and angiopoietin1/2), and the density and structure of capillaries and arterioles (chapter 3 ). To further substantiate the presence of a phase of vascular (chapter 4) and metabolic (chapter 5) remodeling early in the pathogenesis of AF, we studied pigs after 1 week of RAP. In chapter 4, we perform a characterization of responsiveness to various vasodilators and -constrictors in isolated atrial and ventricular small muscular arteries using wire myography. In chapter 5, we have studied mitochondrial structure and a variety 
of key metabolic parameters, both in tissue samples and suspensions of isolated mitochondria. 


\section{REFERENCES}

1. Risk factors for stroke and efficacy of antithrombotic therapy in atrial fibrillation. Analysis of pooled data from five randomized controlled trials. Archives of internal medicine 1994;154:1449-1457.

2. Lip GY, Tean KN, Dunn FG. Treatment of atrial fibrillation in a district general hospital. British heart journal 1994;71:92-95.

3. Kim MH, Johnston SS, Chu BC, Dalal MR, Schulman KL. Estimation of total incremental health care costs in patients with atrial fibrillation in the United States. Circulation Cardiovascular quality and outcomes 2011;4:313-320.

4. Krahn AD, Manfreda J, Tate RB, Mathewson FA, Cuddy TE. The natural history of atrial fibrillation: incidence, risk factors, and prognosis in the Manitoba Follow-Up Study. The American journal of medicine 1995;98:476-484.

5. Go AS, Hylek EM, Phillips KA, Chang Y, Henault LE, Selby JV, Singer DE. Prevalence of diagnosed atrial fibrillation in adults: national implications for rhythm management and stroke prevention: the AnTicoagulation and Risk Factors in Atrial Fibrillation (ATRIA) Study. Jama 2001;285:2370-2375.

6. Schotten U, Verheule S, Kirchhof P, Goette A. Pathophysiological mechanisms of atrial fibrillation: a translational appraisal. Physiological reviews 2011;91:265-325.

7. Wijffels MC, Kirchhof CJ, Dorland R, Allessie MA. Atrial fibrillation begets atrial fibrillation. A study in awake chronically instrumented goats. Circulation 1995;92:1954-1968.

8. Verheule S, Tuyls E, Gharaviri A, Hulsmans S, van Hunnik A, Kuiper M, Serroyen J, Zeemering $\mathrm{S}$, Kuijpers $\mathrm{NH}$, Schotten U. Loss of continuity in the thin epicardial layer because of endomysial fibrosis increases the complexity of atrial fibrillatory conduction. Circulation Arrhythmia and electrophysiology 2013;6:202-211.

9. Verheule S, Tuyls E, van Hunnik A, Kuiper M, Schotten U, Allessie M. Fibrillatory conduction in the atrial free walls of goats in persistent and permanent atrial fibrillation. Circulation Arrhythmia and electrophysiology 2010;3:590-599.

10. Ausma J, Dispersyn GD, Duimel H, Thone F, Ver Donck L, Allessie MA, Borgers M. Changes in ultrastructural calcium distribution in goat atria during atrial fibrillation. Journal of molecular and cellular cardiology 2000;32:355-364.

11. van Bragt KA, Nasrallah HM, Kuiper M, Luiken JJ, Schotten U, Verheule S. Atrial supplydemand balance in healthy adult pigs: coronary blood flow, oxygen extraction, and lactate production during acute atrial fibrillation. Cardiovascular research 2014;101:9-19.

12. van Bragt KA, Nasrallah HM, Kuiper M, van Hunnik A, Kuijpers NH, Schotten U, Verheule S. Dynamic regulation of atrial coronary blood flow in healthy adult pigs. Heart rhythm : the 
official journal of the Heart Rhythm Society 2015;12:991-1000.

13. Gramley F, Lorenzen J, Jedamzik B, Gatter K, Koellensperger E, Munzel T, Pezzella F. Atrial fibrillation is associated with cardiac hypoxia. Cardiovascular pathology : the official journal of the Society for Cardiovascular Pathology 2010;19:102-111.

14. Thijssen VL, van der Velden HM, van Ankeren EP, Ausma J, Allessie MA, Borgers M, van Eys GJ, Jongsma HJ. Analysis of altered gene expression during sustained atrial fibrillation in the goat. Cardiovascular research 2002;54:427-437.

15. Ausma J, Coumans WA, Duimel H, Van der Vusse GJ, Allessie MA, Borgers M. Atrial high energy phosphate content and mitochondrial enzyme activity during chronic atrial fibrillation. Cardiovascular research 2000;47:788-796.

16. Ausma J, Wijffels M, Thone F, Wouters L, Allessie M, Borgers M. Structural changes of atrial myocardium due to sustained atrial fibrillation in the goat. Circulation 1997;96:3157-3163.

17. Maisel WH, Stevenson LW. Atrial fibrillation in heart failure: epidemiology, pathophysiology, and rationale for therapy. The American journal of cardiology 2003;91:2D-8D.

18. Ehrlich JR, Nattel S, Hohnloser SH. Atrial fibrillation and congestive heart failure: specific considerations at the intersection of two common and important cardiac disease sets. Journal of cardiovascular electrophysiology 2002;13:399-405.

19. Li D, Fareh S, Leung TK, Nattel S. Promotion of atrial fibrillation by heart failure in dogs: atrial remodeling of a different sort. Circulation 1999;100:87-95. 



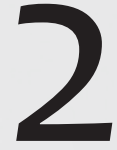

Left atrial oxygen supply in a pig model of congestive heart failure: vascular remodeling and reduced oxygen efficiency

Hussein M Nasrallah; Kelly van Bragt;

Marion Kuiper; Arne van Hunnik;

Ulrich Schotten; Sander Verheule.

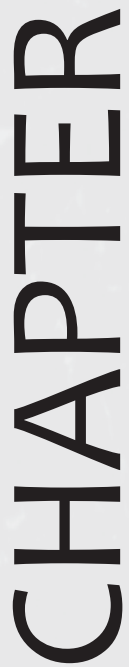




\section{ABSTRACT}

Aims: Congestive heart failure (CHF) increases the risk for atrial fibrillation (AF), and AF exacerbates CHF. We have investigated the left atrial (LA) and left ventricular (LV) coronary vascular regulation, vascular structure and myocardial work in control pigs and pigs with CHF.

Methods and Results: LA and LV coronary vascular conductance and external work were studied in control ( $n=9$ ) and CHF pigs $(n=8)$ (4 weeks of rapid ventricular pacing). In a separate series, oxygen extraction and lactate production were studied in control $(n=7)$ and CHF pigs $(n=6)$. Remodeling of myocardial and vascular structure was studied histologically. CHF significantly decreased LA coronary conductance reserve (2.15 \pm 0.12 vs. $1.44 \pm 0.19 ; P<0.01)$. The response of the LA coronary conductance $\left(C_{L A}\right)$ to pacing was significantly smaller in CHF than in control pigs $(+95 \pm 8$ vs. $+178 \pm 23 \%$ of baseline at 400ms; $\mathrm{P}<0.05)$. The external work performed by the LA was strongly

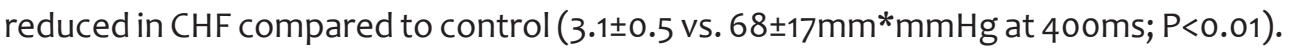
The increase in $C_{L A}$ during 5 minutes of $A F$ showed no significant difference between $\mathrm{CHF}$ and control pigs. During pacing, LA oxygen extraction tended to be lower in $\mathrm{CHF}$ pigs but this was not associated with an increase in atrial lactate production. $\mathrm{CHF}$

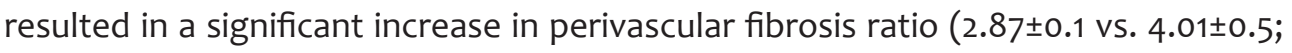
$\mathrm{P}<0.05)$ and a significant decrease in LA capillary density (1767 \pm 538 vs. $1306 \pm 502$ / $\left.\mathrm{mm}^{2} ; \mathrm{P}<0.05\right)$.

Conclusions: CHF leads to remodeling of atrial vascular structure, atrial contractile dysfunction and a marked decrease in atrial oxygen efficiency, potentially contributing to exacerbation of CHF. 


\section{INTRODUCTION}

Clinically, congestive heart failure (CHF) is a major risk factor for atrial fibrillation (AF)' AF occurs in up to $50 \%$ of patients with severe heart failure ${ }^{1}$. The atrial pump function is a predictor for exercise capacity in CHF patients ${ }^{2}$. The loss of the atrial contraction and the irregularventricular response during AF further deteriorate the overall cardiac performance ${ }^{3,4}$, resulting in increased mortality ${ }^{1}$. Structural remodeling of the atria is thought to be an important cause of increased AF stability in $\mathrm{CHF}^{5}$. In a dog model of CHF caused by rapid ventricular pacing (RVP), pronounced and widespread atrial fibrosis was observed, along with degenerative changes in myocytes ${ }^{6}$. These structural abnormalities were far more pronounced in the atria than in the ventricles ${ }^{7}$ and are thought to underlie heterogeneous conduction and increased AF stability ${ }^{8,9}$. However, the exact mechanism responsible for the development of structural remodeling is not clearly understood. A possible explanation could be the development of metabolic dysfunction and/ or ischemia in the atria, as has been reported in dogs after 5 weeks of rapid ventricular pacing ${ }^{10}$.

We have previously shown that in normal healthy pigs, the atrial oxygen supply quickly responds to changing circumstances (such as increased heart rate or AF) by increasing both coronary blood flow and oxygen extraction ${ }^{11,12}$. However, it is unclear whether the regulation of the oxygen supply may be affected by CHF. A small study in dogs with $\mathrm{CHF}^{13}$ has shown a blunted response of atrial flow to adenosine administration and acute AF, suggesting impaired LA vasodilator function. The aim of the present study is to investigate the effect of CHF on atrial and ventricular coronary flow regulation, oxygen extraction and contractile performance. Furthermore, we have assessed alterations in vascular structure that are caused by $\mathrm{CHF}$.

\section{METHODS}

\section{Animal model}

Two groups of adult Dutch Landrace pigs were studied. One group of pigs served as healthy controls. In the CHF group, anesthesia was induced with Zoletil $(5-8 \mathrm{mg} / \mathrm{kg}$ I.M) and Thiopental ( $5-15 \mathrm{mg} / \mathrm{kg}$ I.V). Subsequently, pigs were intubated, mechanically ventilated and anesthesia was maintained with an intravenous infusion of fentanyl (6-12 $\mu \mathrm{g} / \mathrm{kg} / \mathrm{h})$ and Isoflurane 1.0-1.5\%. Under general anesthesia pigs received a right ventricular endocardial pacemaker lead (bipolar screw-in J-lead Medtronic Inc., Minneapolis, Minnesota) connected to an implantable subcutaneous pacemaker (EnPulse, Medtronic Inc., Minneapolis, Minnesota). All animal procedures were in agreement with international standards on research animal use and were approved by the local ethical committee for animal experiments of Maastricht University. 


\section{Induction of CHF}

After one week of recovery from implantation, CHF was induced by three weeks of ventricular pacing at 220bpm at four times threshold, followed by one week of pacing at $180 \mathrm{bpm}$. Ventricular size and function were monitored by echocardiography under sedation (Zoletil, $5-8 \mathrm{mg} / \mathrm{kg}$ ). One echocardiogram was performed before the initiation of ventricular pacing and a second echocardiogram was performed directly before sacrifice experiment to assess the ventricular pump function.

\section{Follow-up experiments}

During sacrifice experiments, anesthesia was induced with Zoletil ( $5-8 \mathrm{mg} / \mathrm{kg}$ I.M) and Thiopental (5-15mg/kg I.V), after which pigs were, intubated, mechanically ventilated and anesthesia was maintained with an intravenous infusion of fentanyl (6-12 $\mu \mathrm{g} /$ $\mathrm{kg} / \mathrm{h}$ ), Midazolam $1.0 \mathrm{mg} / \mathrm{kg} / \mathrm{h}$ and Propofol $0.3 \mathrm{mg} / \mathrm{kg} / \mathrm{h}$. After the measurements, pigs were euthanized by rapid excision of the heart.

\section{Atrial electrophysiology}

To study AF stability and AF activation pattern during sacrifice experiment in control $(n=8)$ and CHF pigs $(n=8)$ an ablation catheter (RFmarinr, Medtronic Inc., Minnesota, MA, USA) was positioned in the right atrium for atrial pacing and AF induction. AF was induced 5 times by $5 \mathrm{~s}$ burst pacing at $20 \mathrm{~Hz}$ and 4 times stimulation threshold in the RA. Sustained AF was defined as a rapid, irregular atrial activation pattern lasting more than 5 min. AF duration was determined as the maximal duration among the five induced episodes. If AF lasted for more than 30min it was terminated by electrical cardioversion. During the AF episodes a high-density circular array with 248 unipolar electrodes (2.4mm inter-electrode distance) was used to record epicardial activation patterns in the atrial free wall. These recordings were analyzed to determine AF cycle length, the number of waves and breakthroughs per cycle using an automated wave mapping algorithm ${ }^{14}$.

\section{Series A Coronary vascular conductance and work index}

In this series, 9 control pigs $(61 \pm 3 \mathrm{~kg})$ were included that were also used in an earlier study ${ }^{12}$. For the present study, all data were reanalyzed by an independent researcher. In addition, 8 pigs with $\mathrm{CHF}(57 \pm 3 \mathrm{~kg})$ were included in this series.

LA and LV coronary vascular conductance and atrial work indices were determined during normal sinus rhythm, fixed pacing rates and acute AF (maintained by atrial pacing at $10 \mathrm{~Hz}$ ). The instrumentation is depicted schematically in figure $1 \mathrm{~A}$.

\section{Series A: Work Indices}

Right and left atrial pressure $\left(P_{R A}, P_{L A}\right)$ and aortic pressure $\left(P_{A 0}\right)$ were measured by Millar microtip pressure catheters. The thermodilution technique was used 
to measure cardiac output. Simultaneous measurements of LV pressure $\left(P_{\mathrm{LV}}\right)$ and ventricular volume were performed using a pressure-volume conductance catheter (Sentron Europe BV, Roden, The Netherlands). Piezoelectric crystals (Sonometrics $2 \mathrm{~mm}$ round crystals, Sonometrics corporation, Ontario, Canada) were implanted in the left atrial free wall to measure changes in LA dimension. The left atrial work index (LAWI) was then calculated as the area within the active part of the pressure distance loop ${ }^{15}$. Similarly, to determine the left ventricular work, $P_{L V}$ was plotted against LV volume. The LV work index (LVWI) was calculated as the area within the resulting LV pressure volume loop.

\section{Series A: Atrial and ventricular coronary blood flow}

Ultrasonic Doppler flow probes (Nano probes PS-Series, Transonic, NY, USA) were placed around an LA and an LV arterial branch originating from left coronary circumflex artery (LCX). At baseline, the coronary vascular conductance was calculated as the coronary blood flow divided by the difference between aortic and right atrial pressure. An intracoronary catheter (JR6 catheter, Cordis Corporation, Bridgewater, NJ, USA) was used for adenosine infusion into the LCx to determine the maximal vascular conductance. The coronary conductance reserve $(C C R)$ was calculated as the ratio between maximal coronary conductance during adenosine

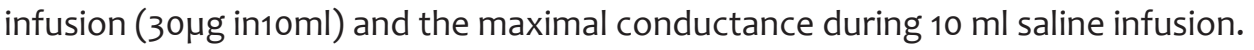

\section{Series B Oxygen extraction and lactate levels}

For series $B, 7$ control pigs $(61 \pm 3 \mathrm{~kg}$ ) were included that were also used in a previous study ${ }^{11}$. An independent researcher reanalyzed data for the present study. In addition, 6 pigs with $\mathrm{CHF}(57 \pm 3 \mathrm{~kg})$ were included. All animals were instrumented as schematically shown in figure $1 \mathrm{~B}$ during sacrifice experiments to study the effect of atrial pacing on LA and LV oxygen extraction levels. In addition, the effect of atrial pacing on LA and LV arterial-venous lactate levels was assessed.

\section{Series B: Instrumentation}

A right atrial pacing catheter, and catheters for $P_{R A}, P_{L V}$ and $P_{A 0}$ were placed as described above in series A. After a left sided thoracotomy, a cannula (Braunule, Braun, Melsungen, Germany) was positioned in a ventricular vein to collect ventricular venous blood samples. Atrial venous samples were collected, as described previously, by creating a pocket within the coronary sinus around a LA vein, while the great cardiac vein was bypassed to the jugular vein". Atrial and ventricular oxygen extraction were calculated as ratio between the arterio-venous oxygen concentration difference and arterial oxygen concentration. Oxygen concentration was measured using an iStat analyzer (Abbott-Laboratories, IL, USA). Samples were also used to determine lactate levels using a Lactate SCOUT+ (Senslab GmbH, Leipzig, 
Germany). Oxygen extraction and lactate concentration were studied during SR and atrial pacing.

A

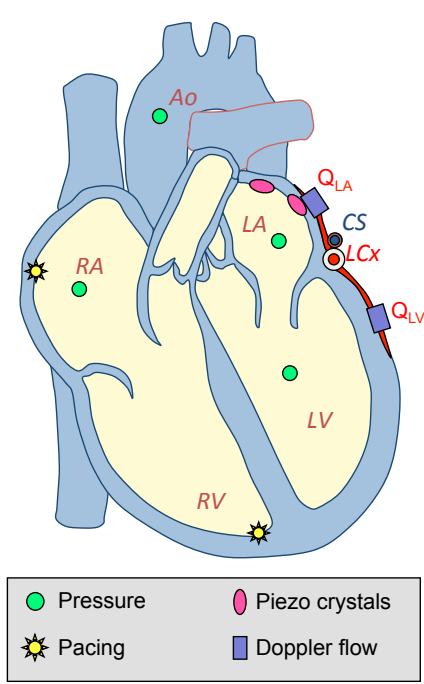

B

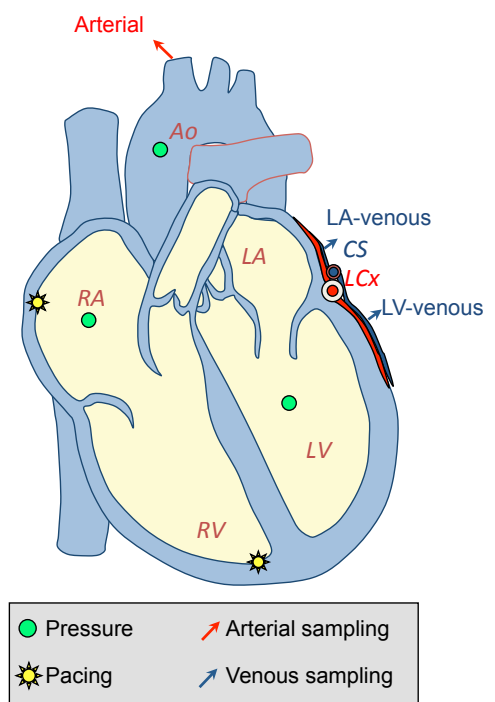

Figure 1. Instrumentation during open-chest experiments.

(A) Series A of experiments performed for determination of coronary blood flow and myocardial work. (B) Series B of experiments performed to quantify the oxygen extraction and lactate levels in arterial samples and in LA and LV venous samples.

\section{Histology and immunohistochemistry}

Samples from the LA and LV were immersed in buffered formalin, embedded in paraffin, sliced into $5 \mu \mathrm{m}$ sections and stained with Sirius red staining for assessment of both overall myocardial fibrosis and perivascular fibrosis ratio (fibrous tissue surrounding arteries/arterioles). For determination of overall fibrosis 3 to 4 locations per Sirius Red-stained slide at 200x magnification were used. For the analysis, the epicardial, endocardial and perivascular fibrous layers were excluded, and a color threshold was set to determine the relative surface area of red pixels (fibrosis) per tissue area determined in percentage. The perivascular fibrosis ratio was calculated as the ratio between area of fibrosis surrounding a vessel and the vessel wall area ${ }^{16}$. Hematoxylin and eosin (H\&E) staining was used to determine myocyte size. The myocyte size was determined from one H\&E section ( 3 to 4 locations per slide) at a 400x magnification in transversely cut cardiomyocytes at the level of the nucleus. To quantify capillary density, sections were stained with Griffonia Simplicifolia I (GSI) staining. Capillary density was calculated as the number of capillaries per surface area (expressed as number $/ \mathrm{mm}^{2}$ ). To determine the capillary density, a magnification of 400x was used. All analysis were blinded, and performed using the Fiji software open source image J package (version 1.49e) 


\section{Statistical Analysis}

Results are expressed as mean \pm SEM. Statistical analysis to compare control and $\mathrm{CHF}$ groups was performed using a linear mixed model using a Bonferroni test for confidence interval adjustment with an SPSS statistics software package (IBM). A p-value smaller than 0.05 was considered to be statistically significant.

\section{RESULTS}

\section{Development of CHF and the substrate for AF}

The development of CHF was evidenced by a significant increase in LV end diastolic pressure and LA pressure, and a decrease in cardiac output and LV ejection fraction (table 1). LV and LA weight were increased significantly by 1.2 and 2 -fold, respectively. At the end of the monitoring period, CHF pigs showed physical signs of end-stage heart failure (lethargy, ascites, cold extremities, loss of appetite and anorexia). The severity of CHF was confirmed by the presence of excess pericardial fluid and by increased lung and liver weights (table 1).

During open chest experiments, AF was induced by burst pacing and the maximal episode duration of AF was measured. CHF animals showed a significantly higher AF episode duration compared to control pigs (figure $2 A$ ). However, no significant difference was observed in the AF cycle length (AFCL) in control and CHF pigs in $L A$. For the RA, the AFCL was significantly higher in CHF pigs (figure2B). The number of waves and breakthroughs per activation cycle did not differ between control and CHF pigs in either atrium (figure $2 C \& D$ ).

\section{Series A: Regulation of coronary vascular conductance}

In each experiment, LA and LV branches at a similar anatomical location along the lateral wall were instrumented with Doppler flow probes. Flow during SR was not significantly different between control and CHF pigs (figure 3A). However, because the atrial weight was higher in the CHF group, the LA flow per atrial chamber weight in the CHF pigs tended to be smaller (figure $3 \mathrm{~B}$ ) In response to adenosine infusion, the CHF group showed a smaller increase in LA flow than control pigs (figure $3 \mathrm{C}$ ), translating into a significantly smaller LA CCR (figure 3D).

Compared to the patterns of LA and LV flow during SR in control pigs, the flow patterns in CHF pigs displayed a weaker fluctuation throughout the cardiac cycle (figure $4 \mathrm{~A}$ and B). This is consistent with a depressed LA and LV contractility in CHF pigs compared to control pigs. To further investigate the regulation of coronary flow in failing hearts, the effect of atrial pacing and acute induction of AF on LA and LV flow was assessed. During pacing at basic cycle lengths of 500ms and $400 \mathrm{~ms}$ (figure $4 C$ ), LA coronary conductance $\left(C_{L A}\right)$ as percentage of $C_{L A}$ during SR increased significantly in control animals. By contrast, pacing at these rates did not increase $\mathrm{C}_{\mathrm{LA}}$ in $\mathrm{CHF}$ pigs. The LV coronary conductance $\left(\mathrm{C}_{\mathrm{LV}}\right)$ as a percentage of $\mathrm{SR}$ showed a 
Table 1. Physical and hemodynamic characteristics of control and CHF pigs (mean \pm SEM).

\begin{tabular}{lll}
\hline Parameter & Control & CHF \\
\hline Body Weight, kg & $61.8 \pm 2.4(n=16)$ & $57.6 \pm 1.9(n=14)$ \\
\hline LVW/BW, g/kg & $2.1 \pm 0.09(n=6)$ & $2.5 \pm 0.03^{* *}(n=7)$ \\
\hline LAW/BW, g/kg & $0.3 \pm 0.04(n=6)$ & $0.6 \pm 0.06 * *(n=7)$ \\
\hline Ejection Fraction, & & Before Pacing $65.6 \pm 9.8(n=8)$ \\
& & After Pacing $26.4 \pm 5.8 \S(n=8)$ \\
\hline CO/BW, I.kg/min & $0.08 \pm 0.009(n=8)$ & $0.05 \pm 0.002 *(n=7)$ \\
\hline LVdP/dtmax, mmHg/min & $1333 \pm 72(n=8)$ & $721 \pm 91^{* *}(n=7)$ \\
\hline LVSP, mmHg & $87.1 \pm 6.0(n=8)$ & $71.91 \pm 4.6(n=7)$ \\
\hline LVEDP, mmHg & $4.7 \pm 0.85(n=8)$ & $11.2 \pm 0.87^{* *}(n=7)$ \\
\hline LA pressure, mmHg & $4.8 \pm 0.87(n=8)$ & $8.7 \pm 0.70 * *(n=7)$ \\
\hline RA pressure, mmHg & $3.1 \pm 0.74(n=8)$ & $10 \pm 0.61 * *(n=7)$ \\
\hline Lung weight $(g / k g)$ & $7.5 \pm 0.3(n=12)$ & $10.2 \pm 0.5(n=11) * *$ \\
\hline Liver weight $(g / k g)$ & $18.2 \pm 0.5(n=12)$ & $29.6 \pm 3.5(n=11) * *$
\end{tabular}

BW: body weight; LVW: LV weight; LAW left atrial weight; CO: cardiac output; LVdp/dtmax is the maximal positive slope of the left ventricular pressure curve; LVSP, left ventricular systolic pressure; LVEDP, left ventricular end diastolic pressure; $P_{L A}$, left atrial pressure; $P_{R A}$, right atrial pressure ** $P<0.01 ; * P<0.05$ CHF vs. Control; $\oint P<0.05$ vs. before pacing

significant increase during pacing in both control and $\mathrm{CHF}$ pigs.

With acute induction of AF (Figure $4 \mathrm{E}$ ), $\mathrm{C}_{\mathrm{LA}}$ in control animals increased significantly. However, in CHF animals (Figure $4 \mathrm{E}$ ), AF did not result in a significant increase in $\mathrm{C}_{\mathrm{LA}}$. $A F$ resulted in a small but significant rise in $C_{L V}$ (Figure $4 \mathrm{~F}$ ) in both control and $\mathrm{CHF}$ pigs.

\section{Series A: Quantification of myocardial work}

To determine the relation of coronary flow to the myocardial work performed, LA and LV pressures and dimensions were recorded. Figure $5 \mathrm{C}$ displays examples of an atrial pressure distance loop of a control and a CHF pig during pacing at a cycle length of 40oms. The area of atrial pressure loops was strongly reduced in CHF pigs compared to control pigs, indicating a pronounced loss of atrial contractility as a result of $\mathrm{CHF}$. The average LAWI in control and CHF pigs during SR and pacing at 500 and 400ms are displayed in Figure 5E. Control pigs showed a strong ratedependent increase in LAWI that was absent in CHF pigs. As expected for failing hearts, ventricular pressure-volume displayed a rightward shift and a smaller area in 

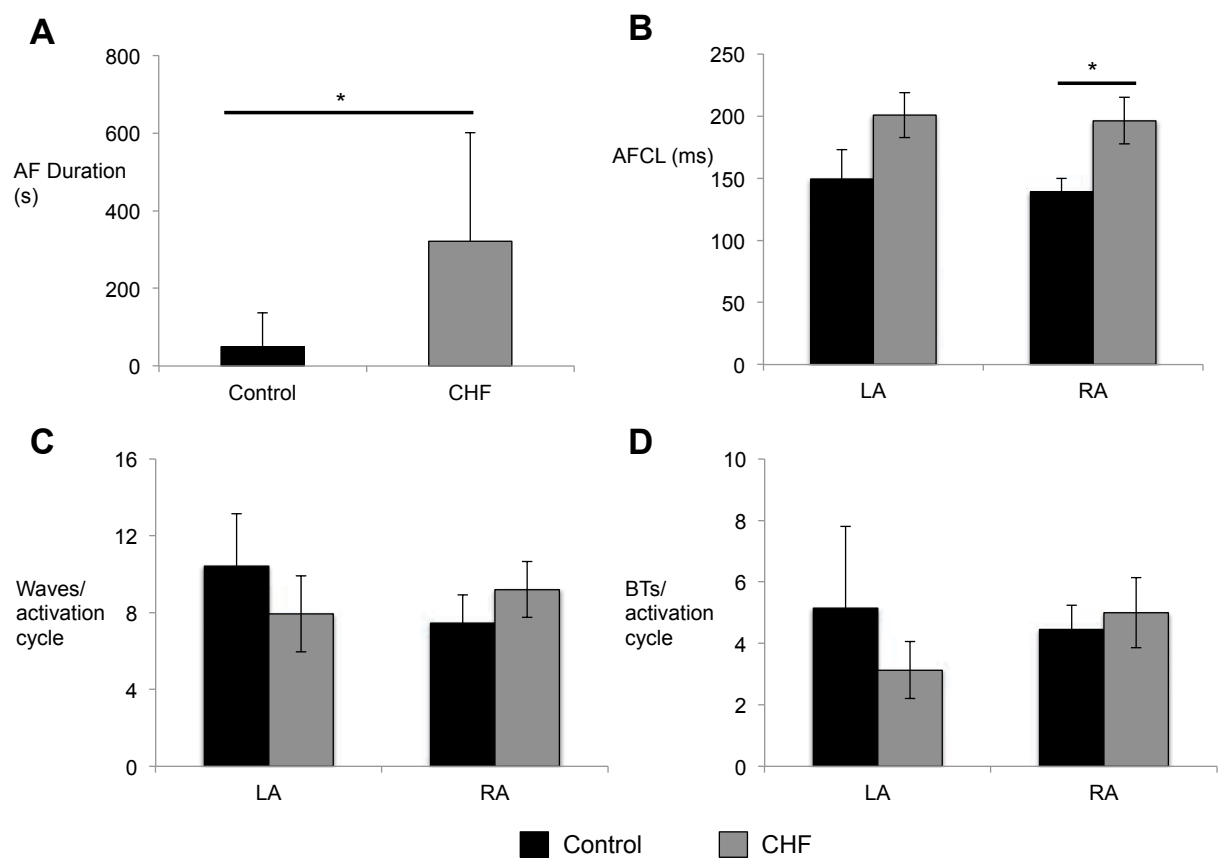

Figure 2. Stability and complexity of atrial fibrillation.

(A) Duration of induced AF episodes in control $(n=13)$ and CHF pigs $(n=8)$. (B) AF cycle length (AFCL) in control $(n=5)$ and CHF pigs $(n=6)$. (C) Number of waves per activation cycle in control $(n=5)$ and $C H F$ pigs $(n=6)$. (D) Number of breakthroughs per activation cycle (Nr. BT/activation cycle) in control $(n=5)$ and CHF pigs $(n=6) . * P<0.05$

CHF compared to control (figure 5D). The left ventricular work index (LVWI) showed a rate-dependent decrease in both groups (figure $5 \mathrm{~F}$ ).

\section{Series B: Oxygen Extraction and lactate production}

In addition to coronary blood flow, myocardial oxygen extraction is a major determinant of the total oxygen delivery. In a separate series of experiments, this parameter was investigated by collecting venous blood samples. The $\mathrm{LAO}_{2}$ extraction during SR was similar in control and CHF pigs (figure 6A). Atrial pacing resulted in a rate-dependent increase in $\mathrm{LAO}_{2}$ extraction in control pigs, but not in CHF pigs. For the LV, the myocardial oxygen extraction was higher during SR than in the LA, and did not increase in either group during atrial pacing (figure 6B).

To investigate whether oxygen supply matched demand during pacing, the difference in lactate concentration between the venous and arterial blood was determined.

The LA arterial-venous lactate difference (figure $6 \mathrm{C}$ ) during SR, and pacing with 500 and 40oms did not show any difference between control and CHF pigs, and did not increase with increasing rate. 
A 8

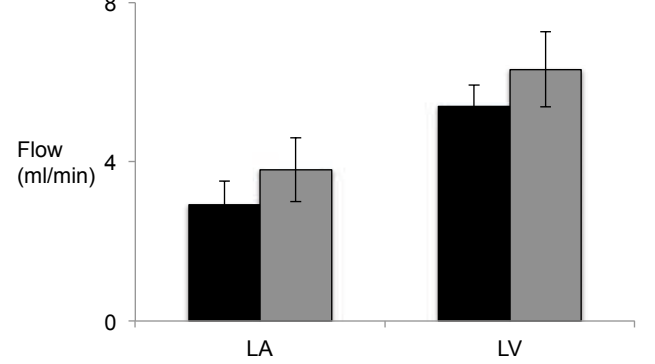

C

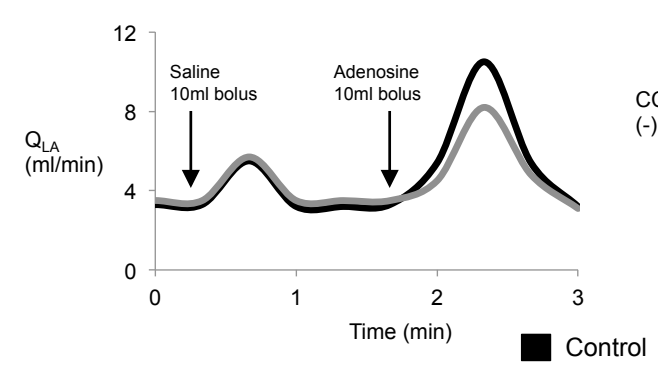

B

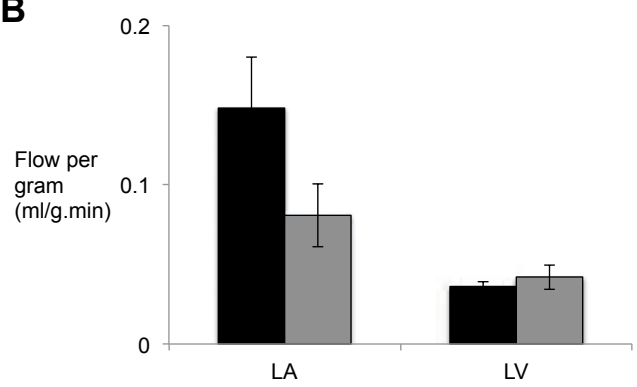

D

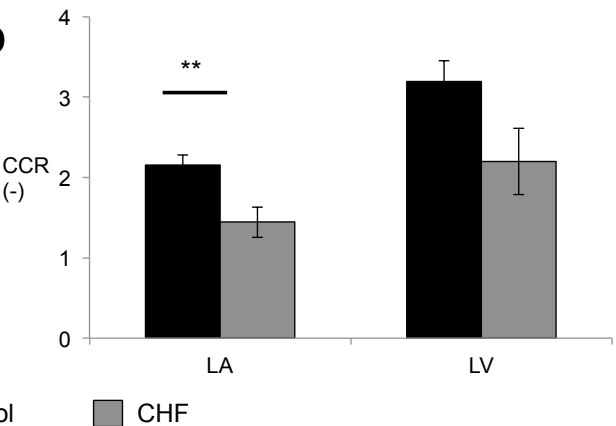

Figure 3. Assessment of coronary blood flow.

(A) Flow during sinus rhythm (SR) in the LA and LV of control $(n=9)$ and CHF pigs $(n=7)$. (B) LA and LV flow per chamber weight in control $(n=9)$ and CHF pigs $(n=6)$. (C) Left atrial flow $\left(Q_{L A}\right)$ during intracoronary infusion of saline and adenosine showed a smaller maximal vasodilation in the CHF group. (D) The coronary conductance reserve (CCR) in the LA and LV of control $(n=8)$ and CHF pigs $(n=5) . * * P<0.01$

\section{Remodeling of myocardial and vascular structure}

Histological sections stained with Sirius Red from the LA from control and CHF pigs (figure $7 \mathrm{~A}$ ) did not show differences between control and CHF pigs in overall myocardial fibrosis, as the percentage of the tissue area occupied by fibrous tissue in the LA and RA, which was not significantly different between control and CHF pigs (figure7A). In the LV, overall myocardial fibrosis was significantly increased in CHF pigs compared to healthy controls (figure $7 \mathrm{~A}$ ). However, histological sections of the LA stained with $\mathrm{H} \& \mathrm{E}$ from control and CHF pigs showed a larger myocyte size in CHF pigs compared to control in both atria (figure $7 \mathrm{~B}$ ).

To determine capillary density, GS-I staining was used. Histological sections from the left atrium showed a smaller number of capillaries per tissue area in CHF pigs compared to control, which was significant for the LA, but not the RA (figure $7 \mathrm{C}$ ). As illustrated in figure $7 \mathrm{D}$, the amount of fibrous tissue surrounding arteries/ arterioles was quantified as perivascular fibrosis ratio. The perivascular fibrosis ratio (the ratio perivascular fibrous tissue area to vessel wall area) was significantly larger in the CHF

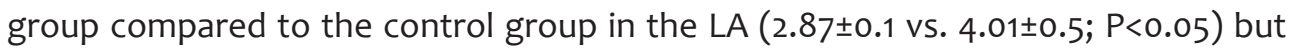
not in the RA. 

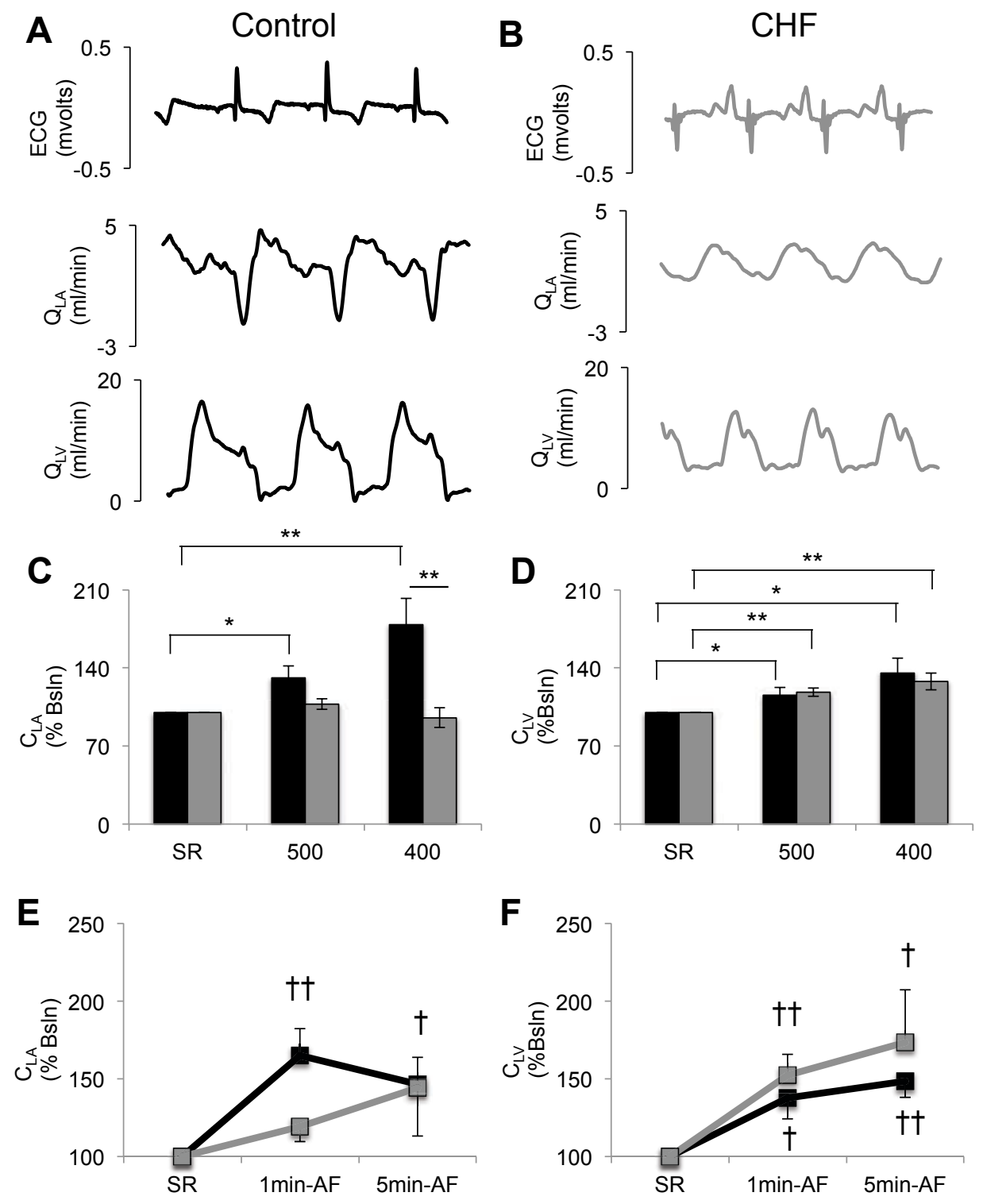

\section{Control $\square \mathrm{CHF}$}

Figure 4. Effects of atrial pacing and AF on coronary blood flow.

$E C G$, left atrial flow $\left(Q_{L A}\right)$ and left ventricular flow $\left(Q_{L V}\right)$ traces recorded in $(A)$ a control pig and $(B)$ a CHF pig. (C) LA coronary conductance $\left(C_{L A}\right)$ during sinus rhythm (SR) and pacing at 500 and $400 \mathrm{~ms}$, all values are normalized to baseline values during $S R$ in control $(n=8)$ and CHF pigs $(n=7)$. (D) LV coronary conductance $\left(C_{L V}\right)$ during $S R$ and pacing at 500 and $400 \mathrm{~ms}$, all values are normalized to baseline values during $S R$ in control $(n=8)$ and CHF pigs $(n=8)$. (E) Effect of 1 and 5 minutes of AF on the $C_{L A}$ in control $(n=7)$ and CHF pigs $(n=6)(F)$. The effect of 1 and 5 minutes of $A F$ on $C_{L V}$ values are normalized to baseline values during $S R$ in control $(n=8)$ and CHF pigs $(n=7) .+P P<0.01$ vs. $S R ;+P<0.05$ vs. SR; ** $P<0.01 ; * P<0.05$ 
A LA $\quad$ B $\quad L V$

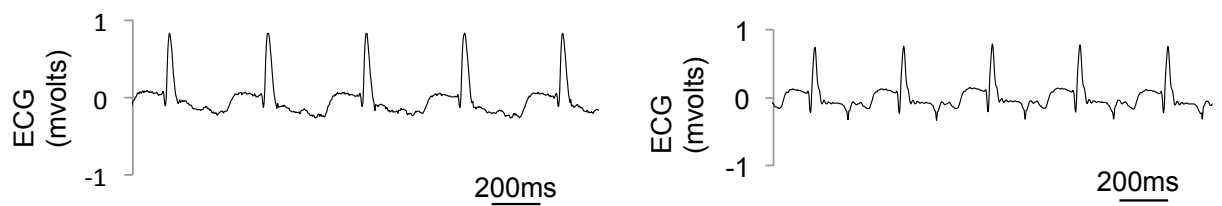

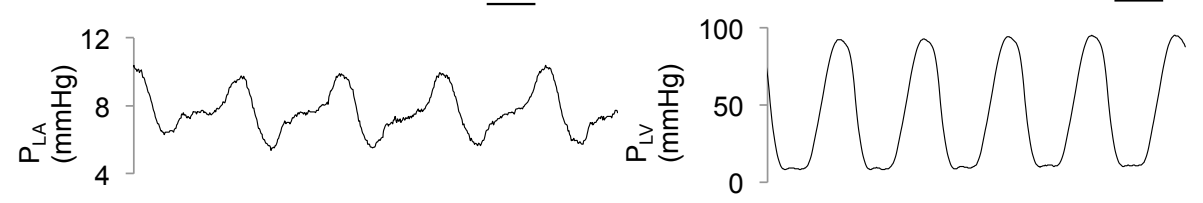

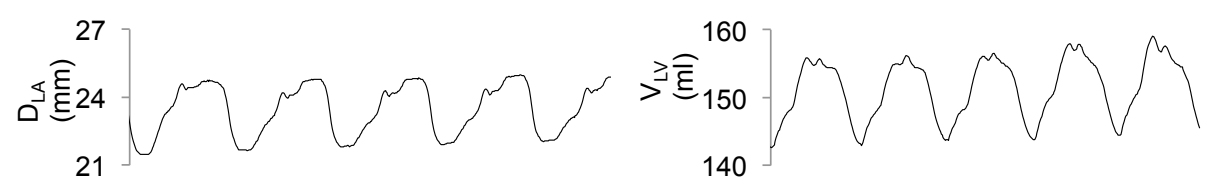
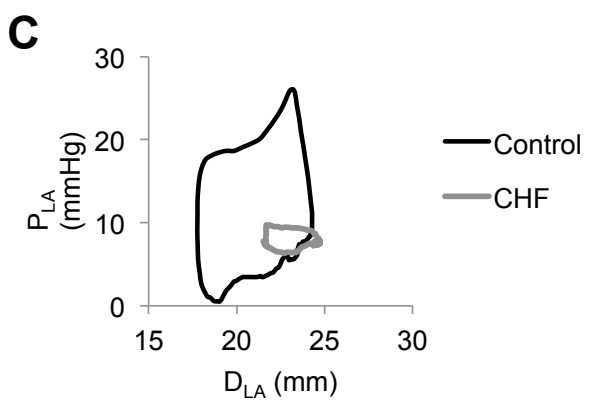

D
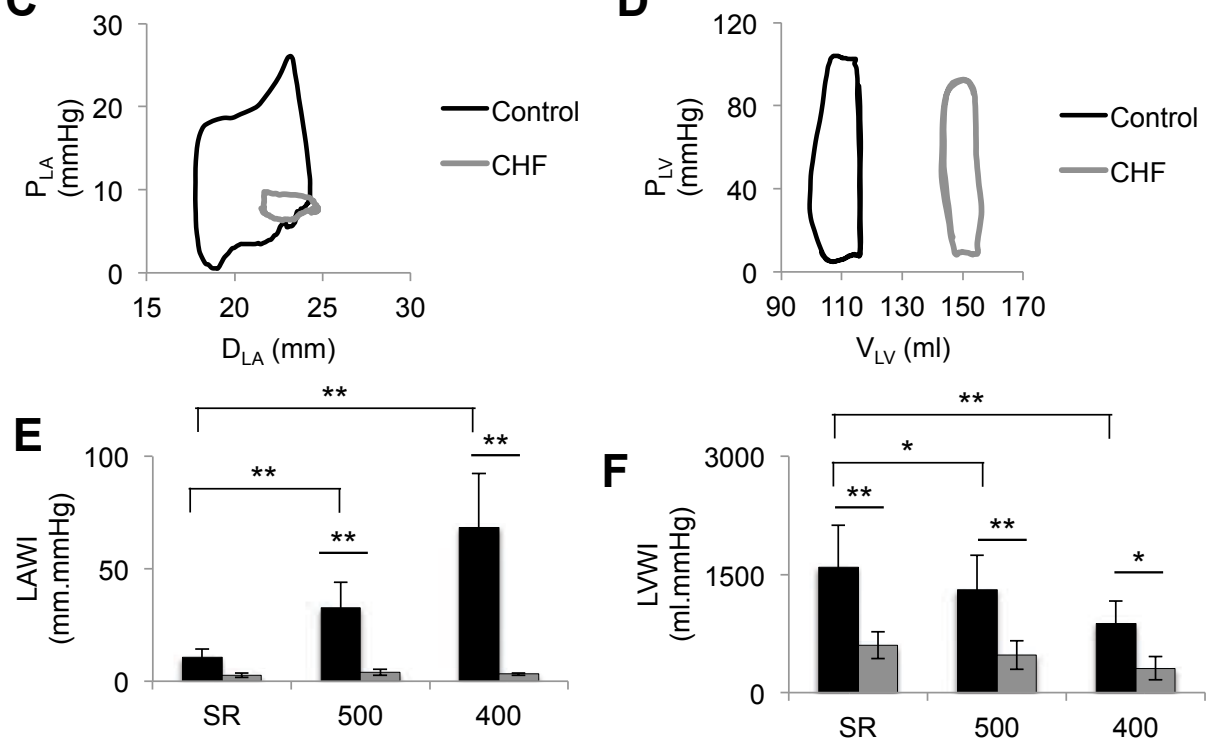

\section{Control $\square \mathrm{CHF}$}

Figure 5. The effect of atrial pacing on myocardial work.

(A) ECG, left atrial pressure $\left(P_{L A}\right)$ and left atrial distance $\left(D_{L A}\right)$ during atrial pacing at $400 \mathrm{~ms}$. (B) $E C G$, left ventricular pressure $\left(P_{L V}\right)$ and $L V$ volume during atrial pacing at $400 \mathrm{~ms}$. (C) $L A$ pressure versus $L A$ diameter during LA pacing at $400 \mathrm{~ms}$ in a control and CHF pig. (D) LV pressure versus LV volume in a control and CHF pig during LA pacing at 40oms. The area of the loops in panels $C$ and $D$ was used to determine the work in index (WI), reflecting the amount of external work performed. (E) Average LA work index (LAWI) during SR and pacing at 500 and $400 \mathrm{~ms}$ in control $(n=8)$ and CHF ( $n=6)$ pigs. (F) Average LV work index (LVWI) during SR and pacing at 500 and $400 \mathrm{~ms}$ in control $(n=9)$ and $\operatorname{CHF}(n=7)$ pigs. ** $P<0.01 ;{ }^{*} P<0.05$ 

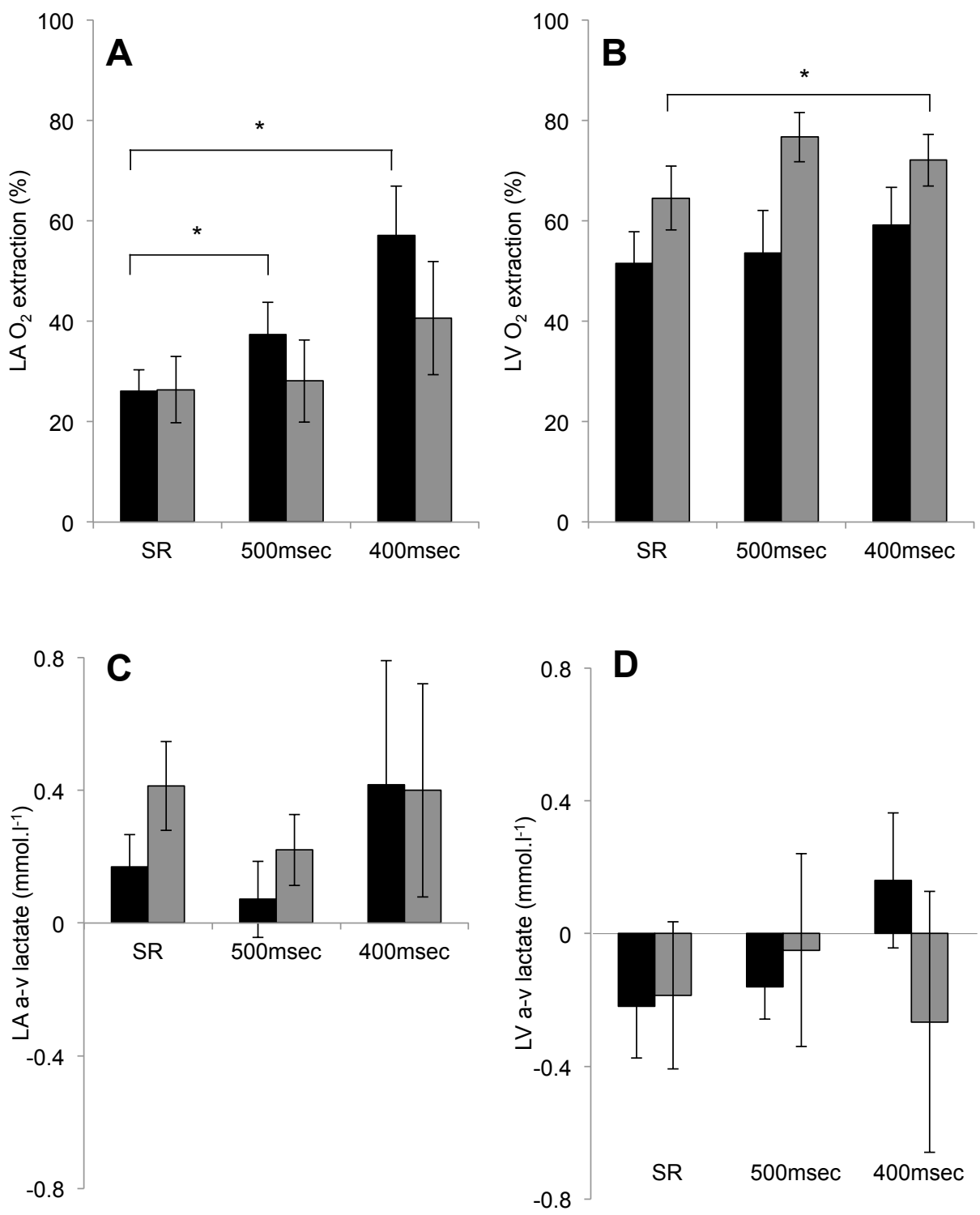

Control

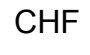

Figure 6. Myocardial oxygen extraction and lactate production.

Percent oxygen extraction during SR and atrial pacing at 400 and $500 \mathrm{~ms}$ for $(A)$ the LA of control $(n=6)$ and CHF pigs $(n=5)(B)$ the LV of control $(n=5)$ and CHF pigs $(n=4)$. (C) Arterial-venous lactate concentration difference during SR and atrial pacing at 500 and $400 \mathrm{~ms}$ for $(C)$ the LA of control $(n=7)$ and CHF pigs $(n=5)$ and $(D)$ the LV of control $(n=5)$ and CHF pigs $(n=4) .{ }^{* *} P<0.01 ; * P<0.05$ 


\section{Control}
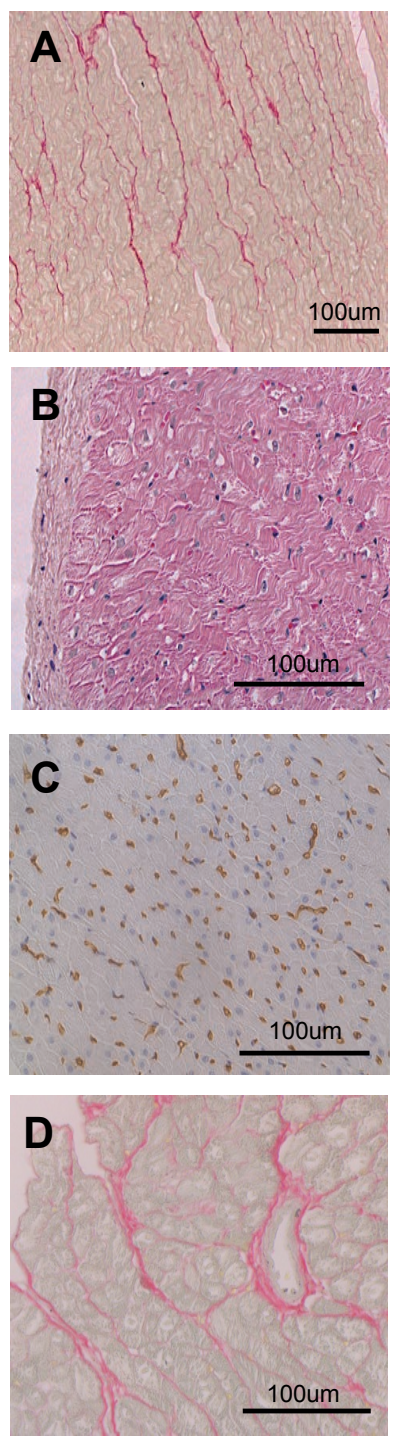

\section{$\mathrm{CHF}$}
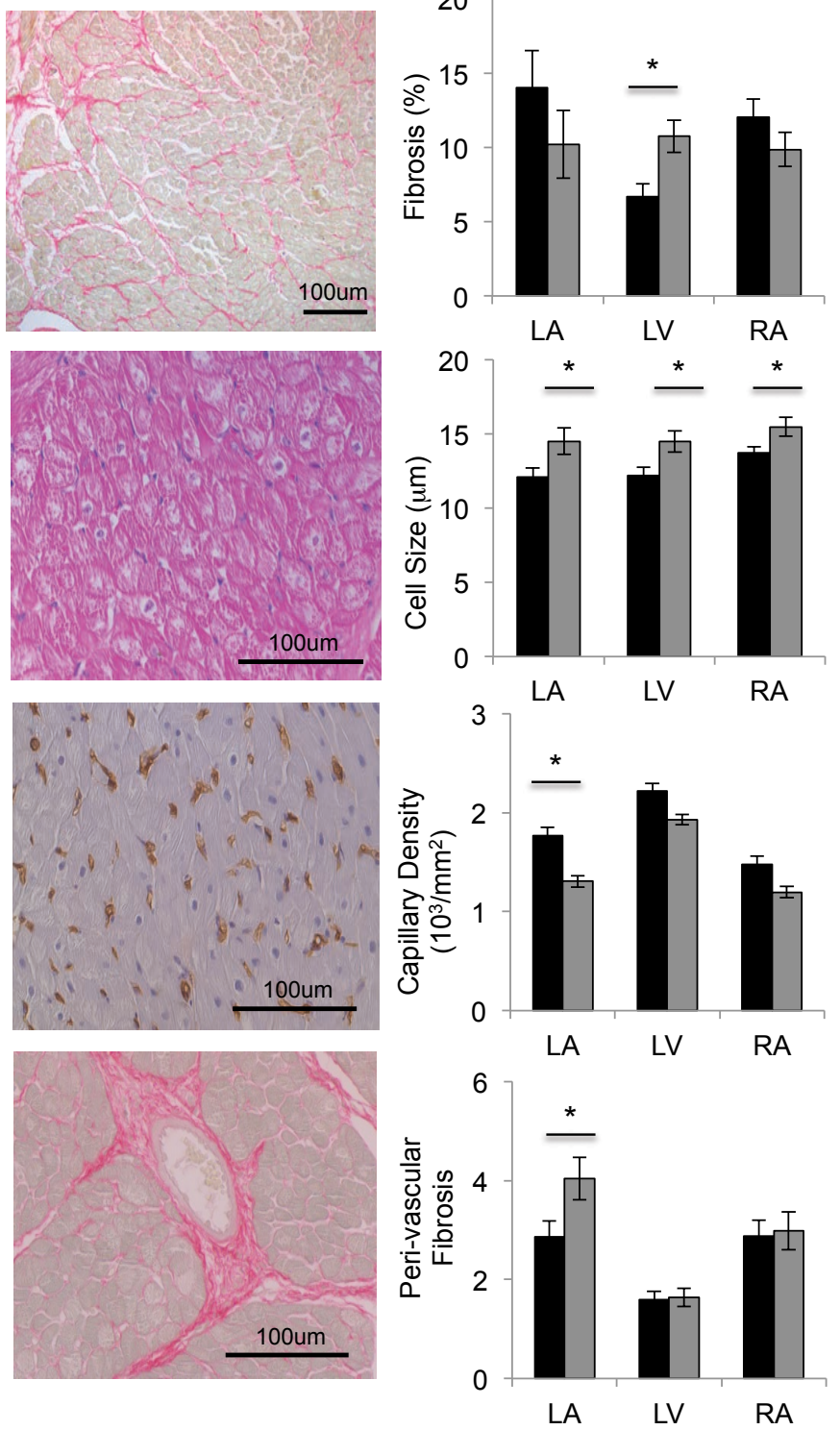

Control

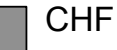

Figure 7. Alterations in myocardial and vascular structure caused by CHF.

Left and middle column shows representative photographs for control and CHF LA, respectively. The right column shows the quantification for the parameter illustrated in that row. (A) Sirius Red staining used to determine the percentage of the tissue area occupied by fibrous tissue in LA, RA and LV of control $(n=10)$ and CHF pigs $(n=12)$. (B) H\&E staining used to determine the myocyte diameter in control $(n=12)$ and CHF pigs ( $n=11)$ (C) GS-I staining was used to assess capillary density, expressed as 1000 s of capillaries per $\mathrm{mm}^{2}$ in control $(n=6)$ and CHF pigs $(n=11)$. (D) Sirius staining used to specifically quantify perivascular fibrosis, expressed as the ratio between perivascular fibrosis tissue area and vessel wall area in control $(n=14)$ and CHF pigs $(n=8)$ 


\section{DISCUSSION}

Our study is the first to characterize left atrial coronary blood supply, contractile performance and vascular remodeling in a model of $\mathrm{CHF}$. CHF in our pig model led to an increased AF stability and atrial cellular hypertrophy. CHF was also associated with a decreased LA coronary conductance reserve and vascular remodeling. Despite the smaller response of LA coronary oxygen supply to higher pacing rates, LA lactate levels did not display signs of oxygen shortage. Atrial contractile performance was strongly reduced and the increase in LA contractile work during atrial pacing was blunted in pigs with CHF.

\section{CHF and the substrate for AF}

Clinically, CHF is associated with increased risk for $\mathrm{AF}^{1}$. Moreover, in a dog model of $\mathrm{CHF}$ the increased stability of $\mathrm{AF}$ is thought to be a result of atrial structural remodeling ${ }^{5}$. In the dog model, $\mathrm{CHF}$ causes pronounced atrial fibrosis ${ }^{6}$. In our pig model, which showed a similar degree of $\mathrm{CHF}$, fibrosis was increased in the LV, but not in the LA. In this context, it may be relevant that the dog model displayed an early (<24hours) and dramatic phase of atrial damage, hallmarked by myocyte necrosis, apoptosis and inflammatory infiltration 7 . The replacement fibrosis observed after 5 weeks might be a result of the early phase of atrial damage, which may not have occurred in our pig model.

We did observe marked atrial dilatation, which is also known to be a strong predictor for AF development ${ }^{17,18}$. Similarly, in a goat model of complete AV block goat, atrial enlargement and myocyte hypertrophy without increased fibrosis were associated with increased AF stability ${ }^{19}$. Mathematical models have also indicated that cellular hypertrophy can lead to discontinuous transverse propagation and thereby lead to increased stability of arrhythmias ${ }^{20}$. Therefore, atrial cellular hypertrophy and dilation may be sufficient to explain the increased AF stability in the pig model.

\section{Coronary conductance reserve and vascular remodeling}

We demonstrate a significant reduction in LA coronary conductance reserve in CHF pigs compared to control pigs. This reduction in conductance reserve will decrease the capacity of the LA to respond to fluctuations in demand. This finding is similar to what was observed in a dog model of atrial hypertrophy resulting from mitral insufficiency and aortic stenosis, in which LA coronary flow reserve was decreased ${ }^{21}$. A small study in dogs with CHF also indicated a reduced LA flow reserve ${ }^{13}$. To assess whether the decrease in coronary conductance reserve was related to vascular remodeling we studied the capillary density and perivascular fibrosis in tissue of control and CHF pigs. CHF pigs showed a marked reduction in the LA capillary density and substantial increase in perivascular fibrosis ratio. Both factors may contribute to the observed reduction in coronary conductance reserve. For the ventricular 
vasculature, a correlation between impaired LV coronary flow reserve and decreased capillary density was demonstrated in patients with cardiomyopathy ${ }^{22}$. Modeling studies further support the role of capillary density as a determinant of the coronary flow reserve ${ }^{23}$. On the other hand, prevention of perivascular collagen formation after myocardial infarction can lead to improvement of myocardial perfusion and coronary flow reserve ${ }^{24}$. Thus, both perivascular fibrosis and capillary rarefaction observed in the atria of pigs with CHF may contribute to the decrease in atrial vasodilator reserve.

\section{Coronary vascular conductance and work index}

With decreased LV pump function, preservation of atrial contractility would help to maintain overall cardiac function by improving the LV diastolic filling, ${ }^{35}$. In the normal heart at rest, the atrial systole contributes between 10 and $15 \%$ of diastolic filling ${ }^{4}$, but its contribution increases during exercise. ${ }^{26,27}$ When ventricular function is decreased as a result of myocardial infarction, cardiomyopathy or heart with preserved LV ejection fraction, this 'booster function' of the atrial contraction also becomes more important (contributing up to an estimated $50 \%$ of cardiac output) ${ }^{4,28-30}$. In patients with myocardial infarction ${ }^{31}$ and heart failure patients with $^{32}$ and without $^{2}$ preserved LV ejection fraction, LA function predicts exercise capacity. A loss of the atrial contraction, such as during $A F$, in these patients may thus contribute to hemodynamic decompensation ${ }^{4}$. However, we have observed that atrial contractility in pigs with CHF was nearly abolished, and by contrast to control animals, did not show the increase during atrial pacing. Dogs with CHF due to 5 weeks of RVP showed a reduction in atrial fractional shortening ${ }^{33}$, but this measure does not allow distinction of passive and active atrial emptying. Atrial contractile dysfunction was also observed during atrial dilation in goats with chronic AV block. There, the decrease in atrial contraction was associated with decrease in the sarcoplasmic reticulum calcium load and atrial myofibrillar remodeling ${ }^{34}$.

In CHF pigs the LA showed a pronounced increase in mass but the atrial blood flow was similar to that observed in control animals. We have recently shown that in normal pigs, atrial pacing caused a rate-dependent increase in LA external work, LA coronary conductance and oxygen extraction ${ }^{11,12}$. By contrast, in CHF pigs neither the LA external work, coronary conductance, nor oxygen extraction increased during pacing. However, the smaller response of LA coronary conductance in $\mathrm{CHF}$ pigs was not associated with an increase in atrial lactate production. These findings indicate that the oxygen supply demand balance in the LA of CHF pigs is preserved during pacing, but that the oxygen efficiency - i.e. the amount of external work performed per amount of oxygen consumed - was dramatically decreased. Although the response of LA supply to changes in rate and rhythm was blunted, we cannot establish cause and effect, in the sense that a deficient atrial supply could 
be responsible for the decrease in LA contractility or that the decreased in atrial contractility obviates the need to increase atrial supply. Nevertheless, the resulting loss of the atrial booster pump function may contribute significantly to reduced exercise capacity and exacerbation of heart failure, even in the absence of AF.

\section{CONCLUSIONS}

This is one of the first studies to assess alterations in atrial vascular structure and function in failing hearts. CHF in pigs was associated with an increased AF stability, atrial cellular hypertrophy and atrial dilatation. The blunted response of LA coronary vascular conductance during atrial pacing in the CHF group was associated with atrial contractile dysfunction. Atrial pacing did not lead to an oxygen supply demand mismatch. However, the oxygen efficiency of the atria was greatly reduced in pigs with CHF.

\section{Limitations}

In this study, we did not inject microspheres to determine the exact blood flow per grams of tissue. As an approximation, the ratio of blood flow per chamber weight was determined. Secondly, due to the complexity of the instrumentation, simultaneously measuring coronary flow and venous sampling from the LA could not be achieved, and was therefore performed in separate series of experiments. Therefore, it was not feasible to exactly determine the myocardial oxygen consumption in order to quantify the myocardial demand. Third, RVP was maintained for 4 weeks in this model and thus, we have only studied one time point in the pathogenesis of CHF and the resulting atrial remodeling. In dogs with CHF due to 5 weeks of RVP showed a marked reduction in ATP and creatine phosphate ${ }^{10}$. Therefore, such a profound reduction in atrial energetic state may lead to a decrease in atrial contractility, similar to what was observed in the hibernating ventricular myocardium due to low flow ischemia ${ }^{35}$. However, we cannot determine whether such depletion in atrial bioenergetics occurs in the pig model. 


\section{REFERENCES}

1. Maisel WH, Stevenson LW. Atrial fibrillation in heart failure: epidemiology, pathophysiology, and rationale for therapy. The American journal of cardiology 2003;91:2D-8D.

2. Donal E, Raud-Raynier P, De Place C, Gervais R, Rosier A, Roulaud M, Ingels A, Carre F, Daubert JC, Denjean A. Resting echocardiographic assessments of left atrial function and filling pressure interest in the understanding of exercise capacity in patients with chronic congestive heart failure. Journal of the American Society of Echocardiography : official publication of the American Society of Echocardiography 2008;21:703-710.

3. Ehrlich JR, Nattel S, Hohnloser SH. Atrial fibrillation and congestive heart failure: specific considerations at the intersection of two common and important cardiac disease sets. Journal of cardiovascular electrophysiology 2002;13:399-405.

4. Bonow RO, Frederick TM, Bacharach SL, Green MV, Goose PW, Maron BJ, Rosing DR. Atrial systole and left ventricular filling in hypertrophic cardiomyopathy: effect of verapamil. The American journal of cardiology 1983;51:1386-1391.

5. Shinagawa K, Shi YF, Tardif JC, Leung TK, Nattel S. Dynamic nature of atrial fibrillation substrate during development and reversal of heart failure in dogs. Circulation 2002;105:2672-2678.

6. Li D, Fareh S, Leung TK, Nattel S. Promotion of atrial fibrillation by heart failure in dogs: atrial remodeling of a different sort. Circulation 1999;100:87-95.

7. Hanna N, Cardin S, Leung TK, Nattel S. Differences in atrial versus ventricular remodeling in dogs with ventricular tachypacing-induced congestive heart failure. Cardiovascular research 2004;63:236-244.

8. Li D, Melnyk P, Feng J, Wang Z, Petrecca K, Shrier A, Nattel S. Effects of experimental heart failure on atrial cellular and ionic electrophysiology. Circulation 2000;101:2631-2638.

9. Verheule S, Sato T, Everett Tt, Engle SK, Otten D, Rubart-von der Lohe M, Nakajima HO, Nakajima H, Field LJ, Olgin JE. Increased vulnerability to atrial fibrillation in transgenic mice with selective atrial fibrosis caused by overexpression of TGF-beta1. Circulation research 2004;94:1458-1465.

10. Cha YM, Dzeja PP, Shen WK, Jahangir A, Hart CY, Terzic A, Redfield MM. Failing atrial myocardium: energetic deficits accompany structural remodeling and electrical instability. American journal of physiology Heart and circulatory physiology 2003;284:H1313-1320.

11. van Bragt KA, Nasrallah HM, Kuiper M, Luiken JJ, Schotten U, Verheule S. Atrial supplydemand balance in healthy adult pigs: coronary blood flow, oxygen extraction, and lactate production during acute atrial fibrillation. Cardiovascular research 2014;101:9-19.

12. van Bragt KA, Nasrallah HM, Kuiper M, van Hunnik A, Kuijpers NH, Schotten U, Verheule S. 
Dynamic regulation of atrial coronary blood flow in healthy adult pigs. Heart rhythm : the official journal of the Heart Rhythm Society 2015;12:991-1000.

13. Hoit BD, Walsh RA, Shao Y, Gabel M, Millard R. Comparative assessment of regional left atrial perfusion by laser Doppler and radionuclide microsphere techniques. Cardiovascular research 1993;27:508-514.

14. Zeemering S, Maesen B, Nijs J, Lau DH, Granier M, Verheule S, Schotten U. Automated quantification of atrial fibrillation complexity by probabilistic electrogram analysis and fibrillation wave reconstruction. Conference proceedings: Annual International Conference of the IEEE Engineering in Medicine and Biology Society IEEE Engineering in Medicine and Biology Society Annual Conference 2012;2012:6357-6360.

15. Schotten U, Duytschaever M, Ausma J, Eijsbouts S, Neuberger HR, Allessie M. Electrical and contractile remodeling during the first days of atrial fibrillation go hand in hand. Circulation 2003;107:1433-1439.

16. Dai Z, Aoki T, Fukumoto Y, Shimokawa H. Coronary perivascular fibrosis is associated with impairment of coronary blood flow in patients with non-ischemic heart failure. Journal of cardiology 2012;60:416-421.

17. Benjamin EJ, Levy D, Vaziri SM, D'Agostino RB, Belanger AJ, Wolf PA. Independent risk factors for atrial fibrillation in a population-based cohort. The Framingham Heart Study. Jama 1994;271:840-844.

18. Eckstein J, Verheule S, de Groot NM, Allessie M, Schotten U. Mechanisms of perpetuation of atrial fibrillation in chronically dilated atria. Progress in biophysics and molecular biology 2008;97:435-451.

19. Neuberger HR, Schotten U, Verheule S, Eijsbouts S, Blaauw Y, van Hunnik A, Allessie M. Development of a substrate of atrial fibrillation during chronic atrioventricular block in the goat. Circulation 2005;111:30-37.

20. Spach MS, Heidlage JF, Dolber PC, Barr RC. Changes in anisotropic conduction caused by remodeling cell size and the cellular distribution of gap junctions and $\mathrm{Na}(+)$ channels. Journal of electrocardiology 2001;34 Suppl:69-76.

21. Bauman RP, Rembert JC, Greenfield JC, Jr. Regional atrial blood flow in dogs. Effect of hypertrophy on coronary flow reserve. The Journal of clinical investigation 1989;83:15631569.

22. Tsagalou EP, Anastasiou-Nana M, Agapitos E, Gika A, Drakos SG, Terrovitis JV, Ntalianis A, Nanas JN. Depressed coronary flow reserve is associated with decreased myocardial capillary density in patients with heart failure due to idiopathic dilated cardiomyopathy. Journal of the American College of Cardiology 2008;52:1391-1398.

23. Jayaweera AR, Wei K, Coggins M, Bin JP, Goodman C, Kaul S. Role of capillaries in 
determining CBF reserve: new insights using myocardial contrast echocardiography. The American journal of physiology 1999;277:H2363-2372.

24. Dedkov El, Zheng W, Christensen LP, Weiss RM, Mahlberg-Gaudin F, Tomanek RJ. Preservation of coronary reserve by ivabradine-induced reduction in heart rate in infarcted rats is associated with decrease in perivascular collagen. American journal of physiology Heart and circulatory physiology 2007;293:H590-598.

25. Mandinov L, Eberli FR, Seiler C, Hess OM. Diastolic heart failure. Cardiovascular research 2000;45:813-825.

26. Channer KS, Jones JV. The contribution of atrial systole to mitral diastolic blood flow increases during exercise in humans. The Journal of physiology 1989;411:53-61.

27. Wright S, Sasson Z, Gray T, Chelvanathan A, Esfandiari S, Dimitry J, Armstrong S, Mak S, Goodman JM. Left atrial phasic function interacts to support left ventricular filling during exercise in healthy athletes. Journal of applied physiology 2015;119:328-333.

28. Rahimtoola SH, Ehsani A, Sinno MZ, Loeb HS, Rosen KM, Gunnar RM. Left atrial transport function in myocardial infarction. Importance of its booster pump function. The American journal of medicine 1975;59:686-694.

29. DeMaria AN, Miller RR, Amsterdam EA, Markson W, Mason DT. Mitral valve early diastolic closing velocity in the echocardiogram: relation to sequential diastolic flow and ventricular compliance. The American journal of cardiology 1976;37:693-700.

30. Phan TT, Abozguia K, Shivu GN, Ahmed I, Leyva F, Patel K, Frenneaux M. Increased atrial contribution to left ventricular filling compensates for impaired early filling during exercise in heart failure with preserved ejection fraction. Journal of cardiac failure 2009;15:890-897.

31. Jikuhara T, Sumimoto T, Tarumi N, Yuasa F, Hattori T, Sugiura T, Iwasaka T. Left atrial function as a reliable predictor of exercise capacity in patients with recent myocardial infarction. Chest 1997;111:922-928.

32. Kusunose K, Motoki H, Popovic ZB, Thomas JD, Klein AL, Marwick TH. Independent association of left atrial function with exercise capacity in patients with preserved ejection fraction. Heart 2012;98:1311-1317.

33. Shi Y, Ducharme A, Li D, Gaspo R, Nattel S, Tardif JC. Remodeling of atrial dimensions and emptying function in canine models of atrial fibrillation. Cardiovascular research 2001;52:217225 .

34. Greiser M, Neuberger HR, Harks E, El-Armouche A, Boknik P, de Haan S, Verheyen F, Verheule S, Schmitz W, Ravens U, Nattel S, Allessie MA, Dobrev D, Schotten U. Distinct contractile and molecular differences between two goat models of atrial dysfunction: AV block-induced atrial dilatation and atrial fibrillation. Journal of molecular and cellular cardiology 2009;46:385-394. 
35. Dispersyn GD, Ausma J, Thone F, Flameng W, Vanoverschelde JL, Allessie MA, Ramaekers FC, Borgers M. Cardiomyocyte remodelling during myocardial hibernation and atrial fibrillation: prelude to apoptosis. Cardiovascular research 1999;43:947-957. 



\section{Atrial fibrillation causes remodeling of the atrial \\ vascular structure}

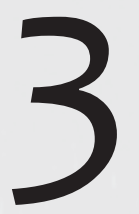

Hussein M. Nasrallah; Kelly A. van Bragt; Marion Kuiper; Will Coumans; Joost Luiken; Dragan Opacic; Anne Marie van OeverenRietdijk; Hetty C. de Boer; Anton Jan van Zonneveld; Ulrich Schotten; Sander Verheule. 


\section{ABSTRACT}

Background: Atrial fibrillation (AF), a common cardiac arrhythmia, is characterized by fast and irregular atrial activation. We have previously shown that acute AF leads to acute supply/demand ischemia. Here, we investigate hypoxic and angiogenic signaling during prolonged AF and the resulting remodeling of vascular structure.

Methods: Healthy control pigs $(n=8)$ were compared to pigs with AF maintained by rapid atrial pacing (RAP, 60obpm) for 1 week (1wRAP, $n=7$ ) or 5 weeks ( $5 w R A P, n=8$ ). Tissue samples were collected from the left atrium (LA) and left ventricle (LV) in all 3 groups. Western blots were performed to quantify expression of Angiopoietin-1 and 2, HIF1a and VEGF. The structure and density of arterioles and capillaries was determined using histology and immunohistochemistry.

Results: 1WRAP and 5WRAP led to a significant increase in HIF1a in comparison to healthy control. VEGF was increased compared to control at 5WRAP, but not at 1WRAP. AF did not affect the levels of angiopoietin-1 and 2. The arteriolar density was not different between control, 1WRAP and 5WRAP. Although the capillary density was not affected at 1WRAP, the 5WRAP was associated with a significant reduction in capillary density compared to healthy controls.

Conclusion: Although hypoxic and angiogenic signaling pathways are activated, AF leads to capillary rarefaction. This phenomenon may restrict the ability of the atria to respond to fluctuations in demand. 


\section{INTRODUCTION}

Atrial fibrillation (AF) is the most common cardiac tachyarrhythmia in clinical practice'. $A F$ itself leads to atrial remodeling processes that contribute to the perpetuation of the arrhythmia. Electrical remodeling is a relatively fast process (1-2days) that involves shortening of action potential duration ${ }^{2,3}$. Over a much slower time course of months to years, structural remodeling gradually develops that increases the complexity of fibrillatory conduction and thereby increasing AF stability ${ }^{4}$. Cellular hibernation $^{5}$ and (endomysial) fibrosis ${ }^{6}$ are hallmarks of structural remodeling, but the underlying causes are not completely understood.

The rapid rates during AF will increase the energy expenditure on ion homeostasis and contraction, leading to an increased atrial oxygen demand, which may not be met by a commensurate increase in oxygen supply. Indeed, we have recently demonstrated that acute AF leads to supply/demand ischemia, as evidenced by lactate production within minutes of AF and a phase of reactive hyperemia after a short AF episode ${ }^{7,8}$. Increased expression of HIF1a has been observed in goats in the first weeks of AF and in AF patients, indicating the ischemic state persists with prolonged $\mathrm{AF}^{9,10}$. In the goat model of $\mathrm{AF}$, this is further supported by a transient decrease in phosphocreatine in the first weeks of $A F^{11}$. On the longer term possible responses to supply/demand ischemia are an increase in supply or a decrease in demand. The phenomenon of atrial myocyte hibernation during $\mathrm{AF}^{5}$, which is similar to that observed in the ventricles during low-flow ischemia5, 12, can be interpreted as an adaptation resulting in a decrease of oxygen demand. An increase in supply could be mediated by changes in both size and density of the vessels perfusing the tissue. In the left ventricle, Dedkov et al. proposed two mechanisms to increase $\mathrm{O}_{2}$ supply: dilation of existing resistance-sized arterioles (Outward remodeling) and angiogenesis, leading to an increase in the density of arterioles and capillaries $^{13}$. In this study, we investigate hypoxic and angiogenic signaling and the remodeling in left atrial and left ventricular vascular density and structure early in the pathogenesis of AF ( 1 and 5 weeks of AF maintained by rapid atrial pacing, RAP).

\section{METHODS}

\section{Animal model}

Three groups of Dutch landrace pigs were studied (50-65kg). One group of pigs served as healthy controls $(n=7)$. In the two other groups, AF was maintained by rapid atrial pacing for 1 week (1WRAP, $n=7$ ) or 5 weeks ( $5 \mathrm{WRAP}, \mathrm{n}=7$ ). RAP pigs received a right atrial endocardial pacemaker lead (J-Leads Capsurefix 5568 - 53 cm, Medtronic Inc. Minneapolis, Minnesota) connected to an implantable subcutaneous pacemaker (Itrel II, Medtronic, Minneapolis, MN). All animal procedures were in agreement with international standards on research animal use and were approved 
by the local ethical committee for animal experiments of Maastricht University.

\section{Induction of AF}

After one week of recovery from pacemaker implantation, the pigs were sedated with an intramuscular injection of Zoletil $(5-8 \mathrm{mg} / \mathrm{kg})$. AF was maintained by rapid atrial pacing at $600 \mathrm{bpm}$ with four times pacing threshold. To prevent the development of heart failure in the 5WRAP group, the ventricular rate was controlled by digoxin $10 \mu \mathrm{g} / \mathrm{kg}$ for 1 week, followed by $5 \mu \mathrm{g} / \mathrm{kg}$ for 4 weeks. Digoxin was discontinued 3 days before the sacrifice experiment in order to reach plasma levels $<0.5 \mu \mathrm{g} / \mathrm{ml}$.

\section{Follow-up experiments}

For follow-up experiment animals were sedated with Zoletil (5-8mh/kg I.M.). In the RAP groups, pacing was stopped immediately prior to the induction of anesthesia and restarted directly after induction. Anesthesia was maintained with fentanyl $(6 \mu \mathrm{g} /$ $\mathrm{kg} / \mathrm{h})$, midazolam $(0.8 \mathrm{mg} / \mathrm{kg} / \mathrm{h})$ and propofol $(0.3 \mathrm{mg} / \mathrm{kg} / \mathrm{h})$. The heart was excised via a lateral incision. Tissue samples were collected from the LA and LV, snap frozen in liquid nitrogen and stored at $-80^{\circ} \mathrm{C}$ for Western blot and immunohistochemistry. Additional tissue samples were stored in formalin for histological analysis.

\section{Immunohistochemistry}

Samples fixated in formalin were embedded in paraffin and sliced into $5 \mu \mathrm{m}$ thick sections. To determine the capillary density, sections were stained with Griffonia Simplicifolia I (GSI) and photographed at a magnification of 400x. Detection of capillaries performed as shown in figure 1 using trainable Weka segmentation algorithm that is part of the open source Fiji software (www.fiji.sc). The segmentation algorithm had first been trained to reliably recognize capillaries based on color and shape. The trained algorithm was then used to segment each image into capillaries, myocytes and background. The segmented images were converted to a grayscale and thresholded to visualize only capillaries. Subsequently, a 'particle analysis' was performed to determine the number and size distribution of those capillaries. For the size distribution, only objects with a circularity greater or equal to 0.4 Yes I checked that and we used this method were included, in order to exclude longitudinally sectioned vessels. Capillary density was calculated as the number of capillaries per surface area (expressed as number $/ \mathrm{mm}^{2}$ ).

To determine the arteriolar density and size distribution, frozen tissue samples were sectioned into $5 \mu \mathrm{m}$ sections. Arterioles were labeled using an anti $\alpha$-smooth muscle actin antibody (A2547, Sigma Aldrich, St Louis, MO). The quantification of number and size was performed by setting a color threshold to determine the positively labeled arterioles (green pixels). 

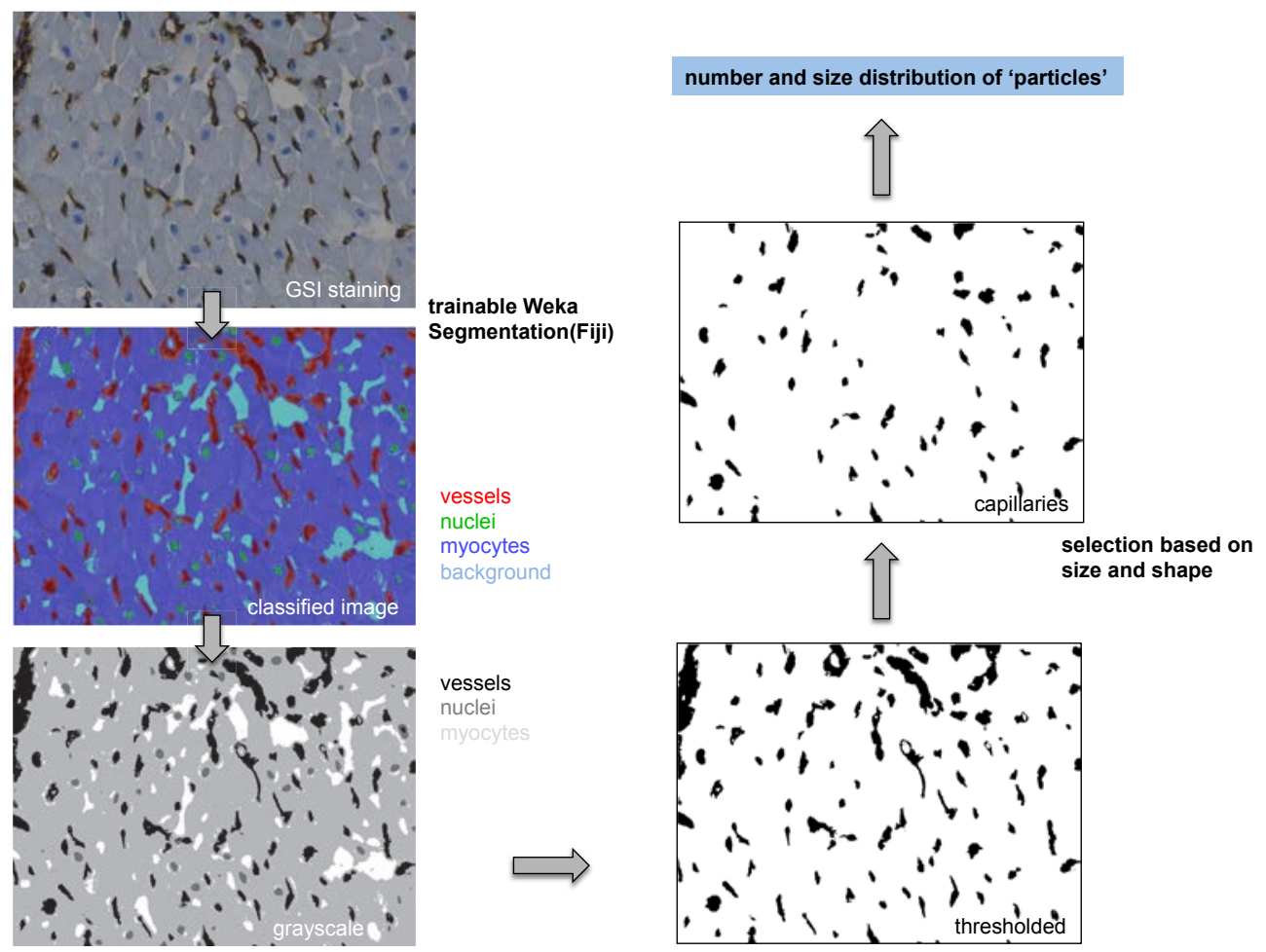

Figure 1. Quantification of capillary density.

Successive steps in this method are: (A) Original image of staining with GS-I. (B) Color-coded image segmented based on color and shape of objects. (C) Image converted to gray scale. (D) Black and white image after thresholding. (E) For determination of the number of capillaries, objects smaller than a minimum size (i.e.) and larger than a maximal size (i.e. arteries and veins) were excluded. For determination of capillary size, an additional criterion excluded non-circular objects.

\section{Western blotting}

Proteins were separated by SDS-PAGE on 4-12\% Bis-Tris Standard XT precast gels (Bio-Rad) and moved to nitrocellulose membranes. The membranes were probed with the appropriate primary antibodies HIF1a (Santa Cruz, H-206, sC-10790), VEGF (NOVUS, VG76e), Ang1 (Santa Cruz, N-18, sc-6319), Ang2 (Santa Cruz, F-18, sc-7017) with 1:1000 dilutions in $5 \%$ bovine serum albumin. The protein bands were visualized using enhanced chemiluminescence, and the immunoblot intensities were quantified using Bio-Rad Quantity One.

\section{Statistical analysis}

Results are expressed as mean \pm SEM. Statistical analysis was performed using a linear mixed model with a Bonferroni correction using a statistical software package SPSS (IBM). A p value less than 0.05 was considered to be statistically significant. 


\section{RESULTS}

\section{HIF1a and VEGF}

Representative blots from LA displayed higher HIF1a content in the 1WRAP and 5WRAP groups compared to control (figure 2A). Quantification of the relative band intensity of HIF1a demonstrated a significant increase in the HIF1a expression in both the 1WRAP and 5WRAP LA tissue compared to healthy controls (Figure2B). In the LV, HIF1a was significantly increased in the 1WRAP group, but not in the 5WRAP group. Representative blots showed higher VEGF expression only in the 5WRAP group of pigs compared to control (figure $2 \mathrm{~A}$ ). The relative band intensity for VEGF showed no statistical difference either for the LA or LV at the 1-week timepoint. At the 5-week timepoint, the expression of VEGF was significantly increased in the LA, but slightly decreased in the LV (figure $2 \mathrm{C}$ ).

A

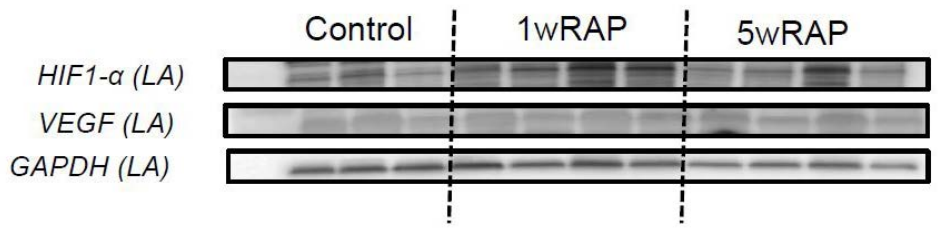

B

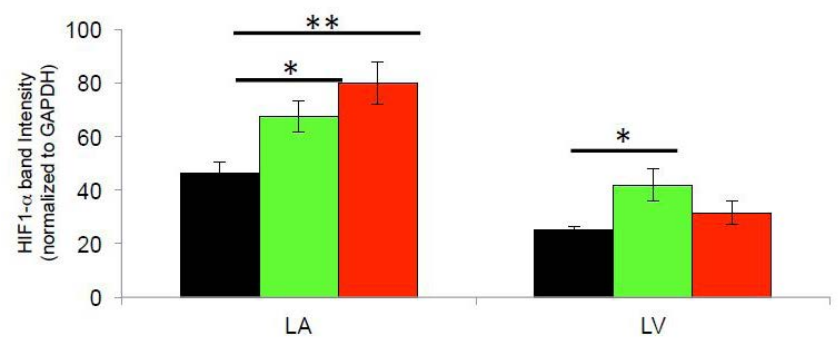

C

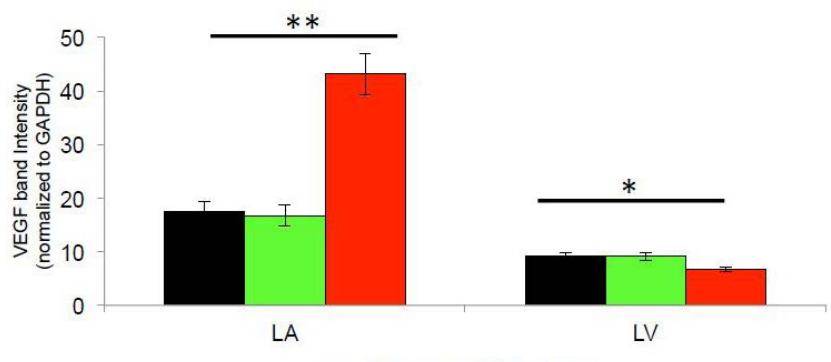

Figure 2. Hypoxia and angiogenesis markers.

(A) Western blots displayed an increase in the levels of HIF1- $\alpha$ in the LA of 1WRAP $(n=7)$ and $5 W R A P(n=8)$ compared to control $(n=7)$. (B) Quantified band intensity of HIF1- $\alpha$ normalized to GAPDH content in the LA and LV. (C) Western blots displayed similar levels of VEGF in the LA of 1WRAP $(n=7)$ but increased levels in the $5 W R A P(n=8)$ compared to control $(n=7)$. (D) Quantified band intensity of VEGF normalized to GAPDH content in the LA and LV. Values are expressed as mean \pm SEM. ${ }^{*} P<0.05$ vs. control; $* * P<0.01$. 


\section{Arteriolar density and size distribution}

Representative immunofluorescence images of staining with a-smooth muscle actin (arteries and arterioles with smooth muscle cells) and DAPI (nuclei) are shown figure $3 A, B \& C$ ). Quantification of the LA and LV arterioles density did not reveal any statistical difference in arteriolar density in the 1WRAP and 5WRAP pigs compared to healthy control for either the LA or LV (figure 4A). The distribution of arteriolar sizes in the LA and LV did not indicate any change in the shape of the histograms between groups (figure $4 C \& D$ ), and the median arteriolar size was not different between the groups(figure 4B).
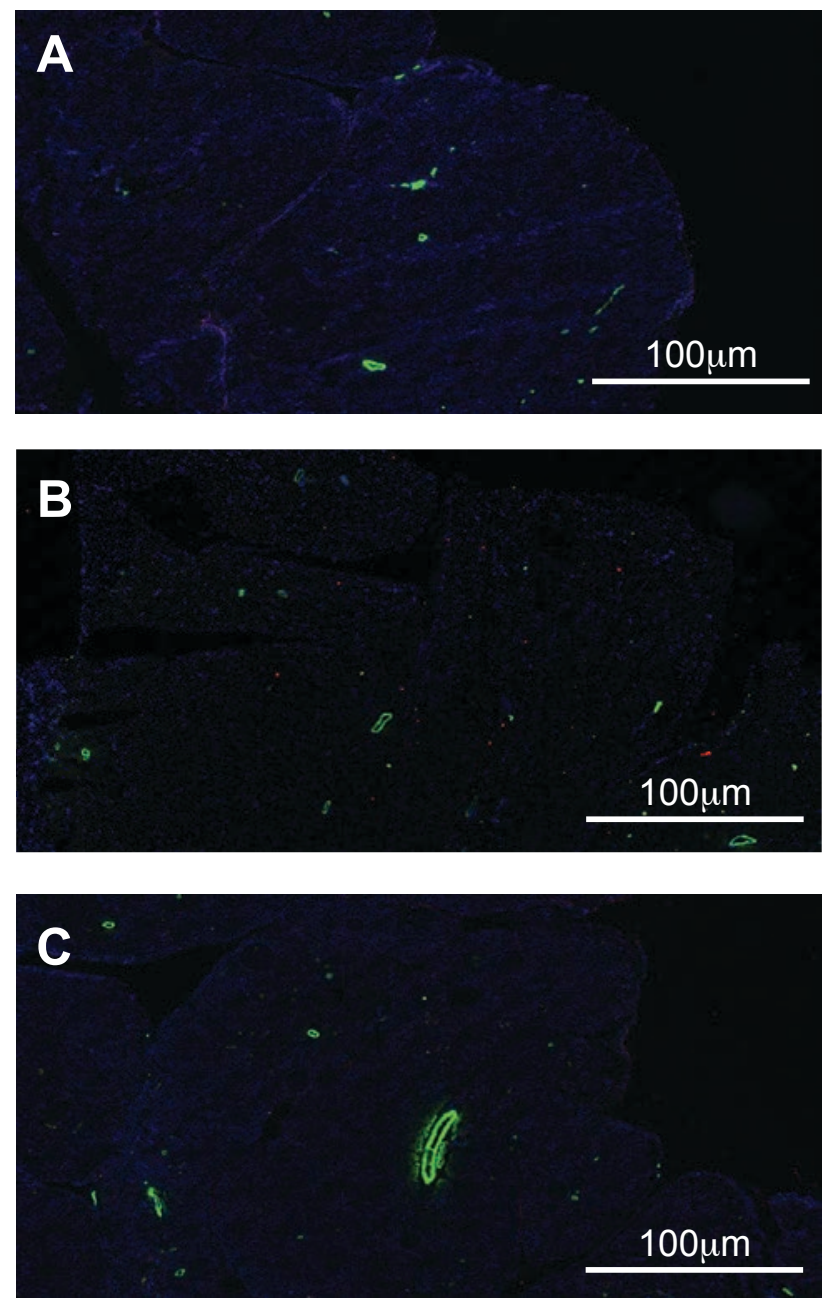

Figure 3. Immunofluorescent staining of arterioles.

Representative immunofluorescence images of a-SMA (smooth muscle cells) and DAPI (nuclei) labeling at 40X magnification from LA tissue of (A) control pigs, (B) 1WRAP pigs and (C) 5WRAP. 
A

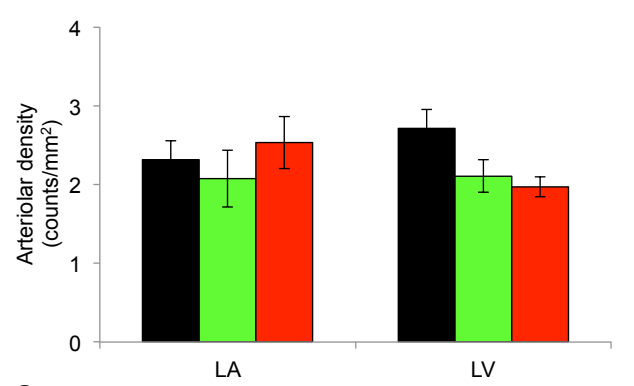

C

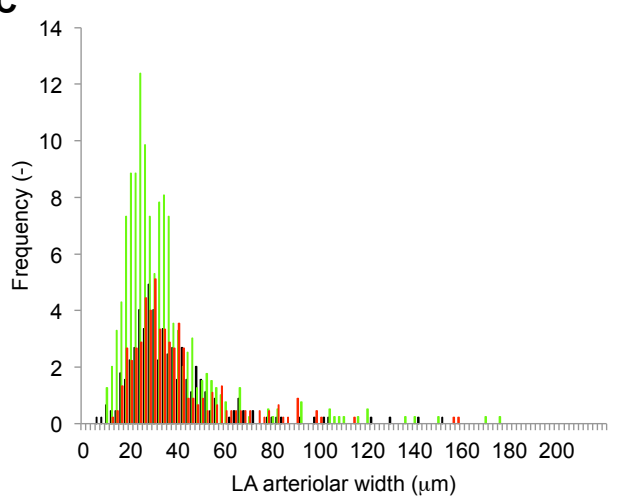

B

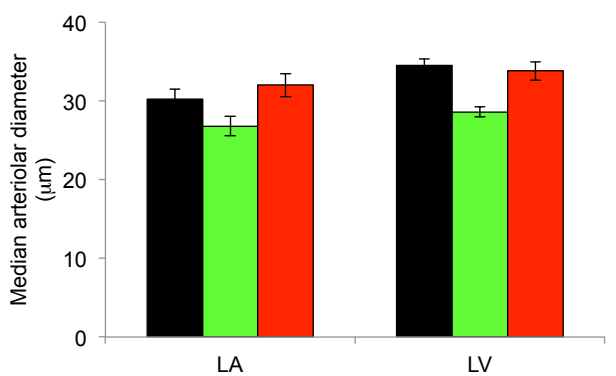

D

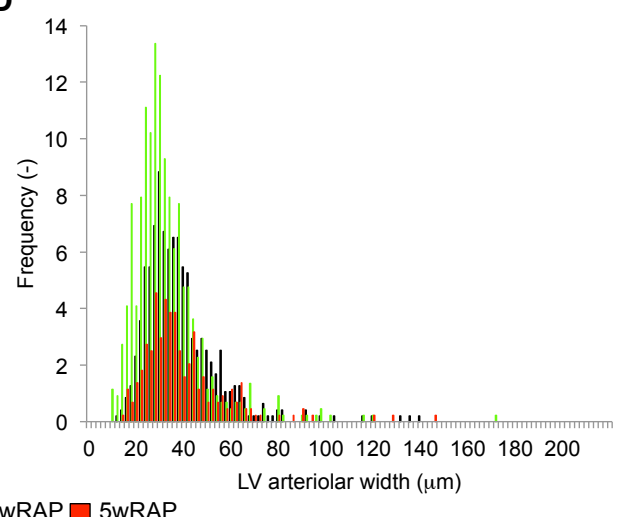

Figure 4. Arteriolar density and size distribution.

(A) The arteriolar density, representing the number of arterioles per $\mathrm{mm}^{2}$. (B) Median arteriolar size in $(\mathrm{mm})$ of LA and LV of control $(n=7), 1 W R A P(n=7)$ and 5 WRAP $(n=8)$ groups. Values are expressed as mean \pm SEM. Histograms of the distribution of the arteriolar size in control, 1WRAP and 5 WRAP pigs in (C) the LA and (D) the LV.

\section{Capillary density and size}

Representative GS-I immunohistological sections from the LA indicated that there were fewer capillaries per $\mathrm{mm}^{2}$ in the 5 wRAP group (figure $5 \mathrm{~A}, \mathrm{~B} \& \mathrm{C}$ ). Quantification of the capillary density was performed as illustrated in figure 1. Compared to control, the capillary density was not altered in the 1WRAP group, but it was significantly decreased in the 5WRAP group (figure 6A). In the LV, capillary density was not significantly different between the groups (figure 6A). The LA capillary size distribution exhibited a leftward shift towards smaller capillary size in both the 1WRAP and the 5WRAP group. The median capillary diameter was significantly smaller in the 1WRAP group, indicating a more abundant number of small capillaries (figure $6 \mathrm{~B} \& \mathrm{C}$ ). In the LV, the distribution showed a shift towards smaller capillary size after 1 week of RAP, but not after 5 weeks of RAP (figure 6D). Moreover the 5 WRAP group displayed a larger median capillary diameter in comparison to control and 1WRAP (figure6D). 

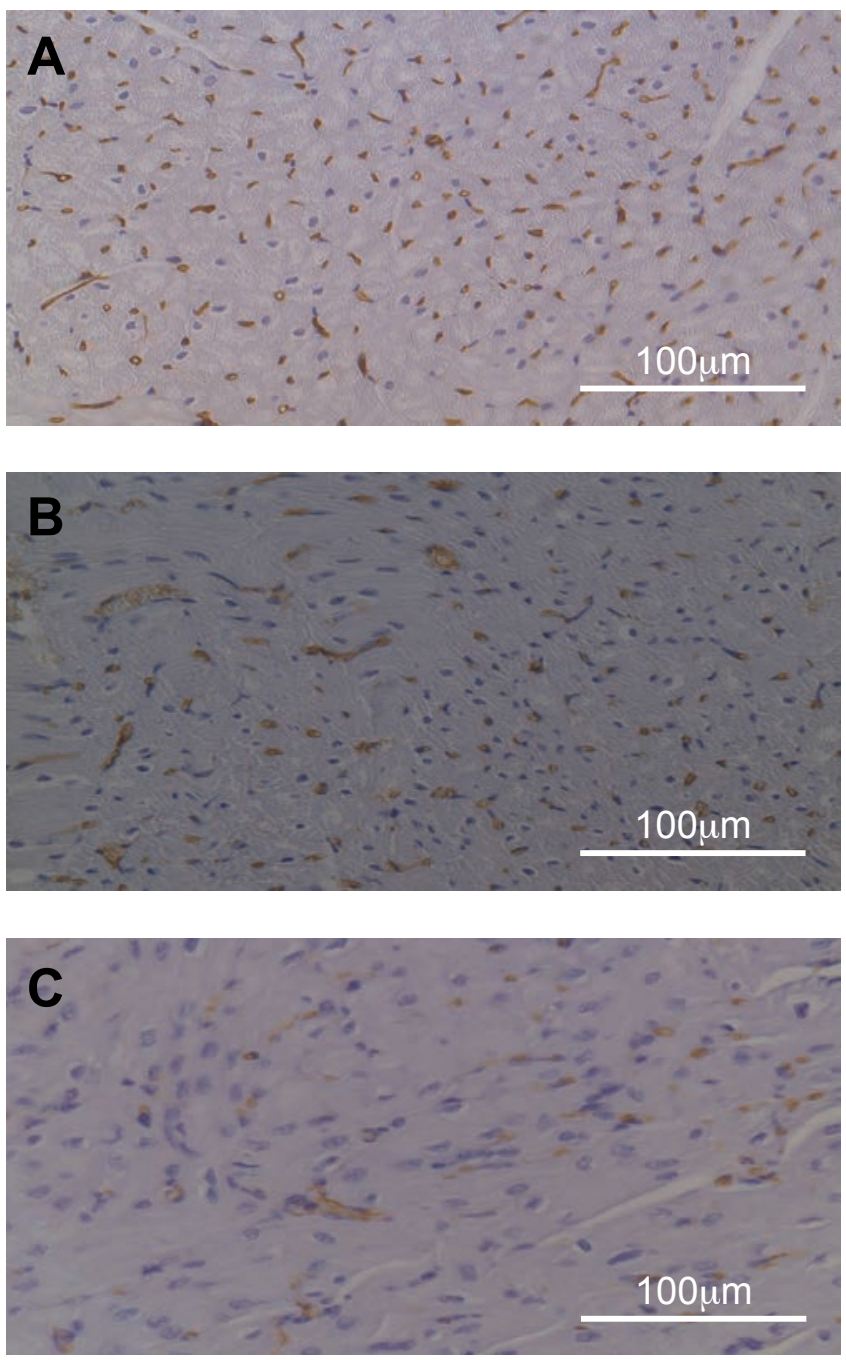

Figure 5. GSI staining of capillaries.

Representative image at 400X magnification showing GSI labeling in the LA of (A) a control pig (B) a 1WRAP pig and (C) a 5WRAP pig. 


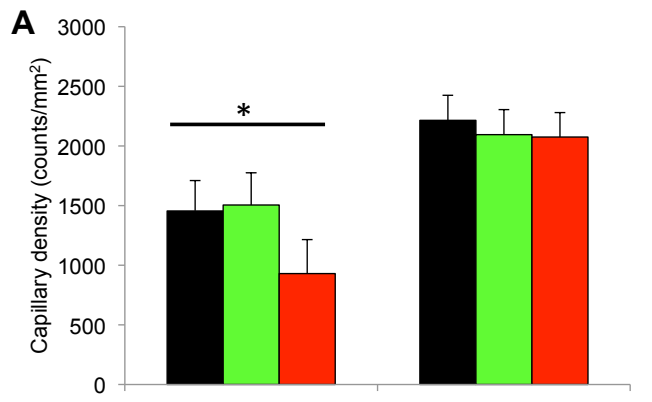

LA

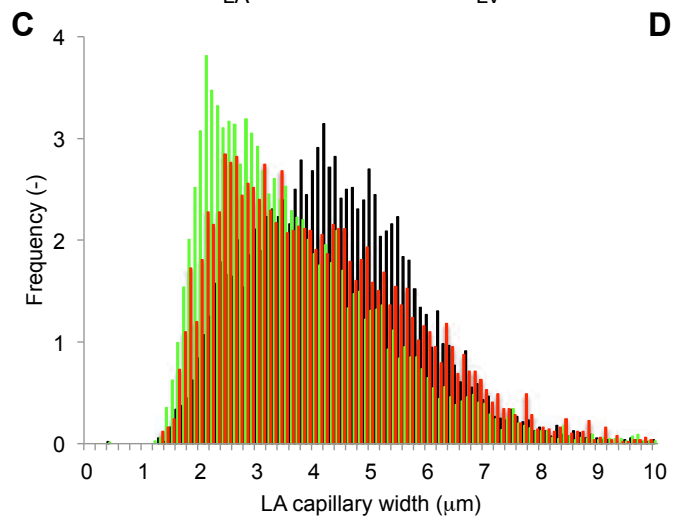

- Cont $\square$ 1wRAP $\square$ 5wRAP
B
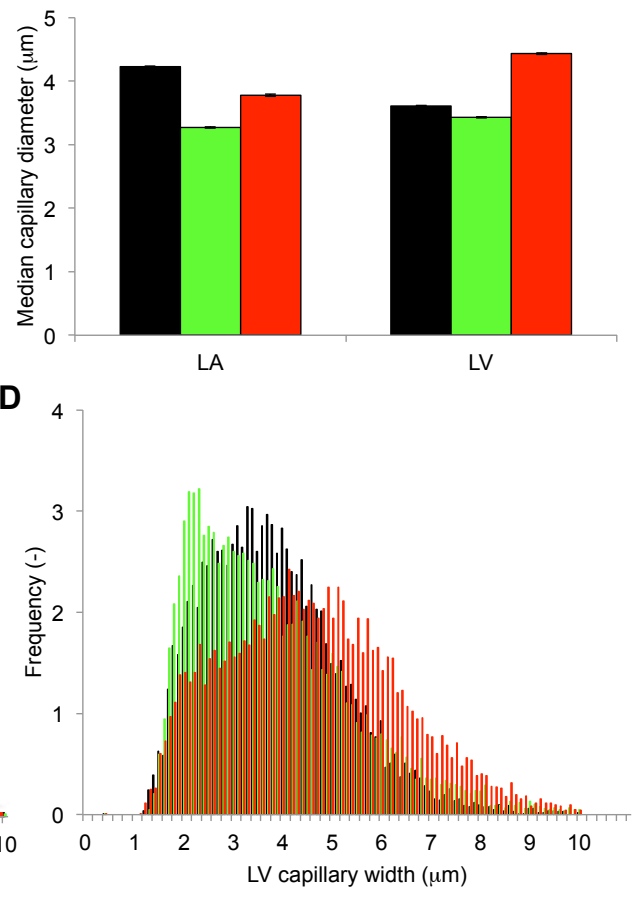

Figure 6. Capillary density and size distribution

(A) Capillary density, expressed as the number of capillary per $\mathrm{mm}^{2}$ in the LA and LV of control $(n=7)$, 1WRAP ( $n=7)$ and 5WRAP ( $n=8)$ groups. (B) Median capillary size in the LA and LV of control $(n=7)$, 1WRAP $(n=7)$ and $5 W R A P(n=8)$ groups. Values are expressed as mean $\pm S E M .{ }^{*} P<0.05$ vs. control. Histograms depicting the capillary size in control, 1WRAP and 5WRAP pigs for (C) the LA and (D) the LV.

\section{Angiopoietin 1 \& 2}

The stability of newly formed vessels is, among other factors, affected by the balance between the expression of Angiopoietin1 (Ang-1) and Angiopoietin 2 (Ang2). Figure 7 shows representative immunohistochemistry images of Ang1 and Ang2 staining (labeled in brown) counter stained with Hematoxylin for labeling the nuclei in the LA of control, 1WRAP and 5WRAP(Figure7A\&B). Western blots of Ang-1 and Ang-2 did not indicate differences in band intensity in the 1WRAP and 5WRAP groups compared to control (figure $8 \mathrm{~A}$ ). The relative band intensities for both Ang1 and Ang2 normalized to GAPDH content did not show any significant changes for either the LA or LV between the groups (figure $8 \mathrm{~B} \& \mathrm{C}$ ). 


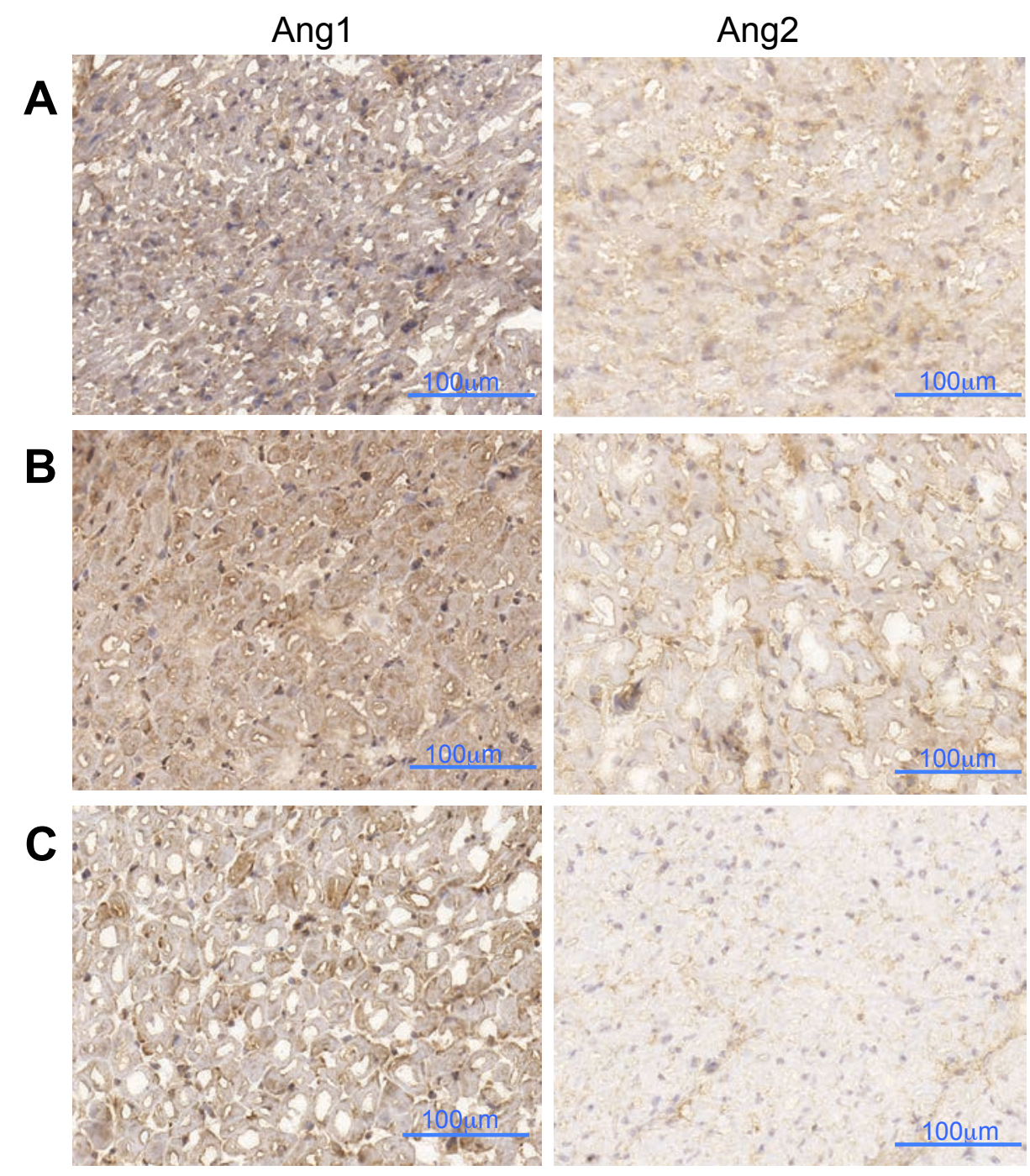

Figure 7. Distribution pattern of angiopoietin1 (Ang1) and angiopoietin2 (Ang2). Examples shown for Ang1 (left panels) and Ang2 (right panels) in the LA of (A) a control pig, (B) a 1WRAP pig and (C) a 5 WRAP pig. 
A

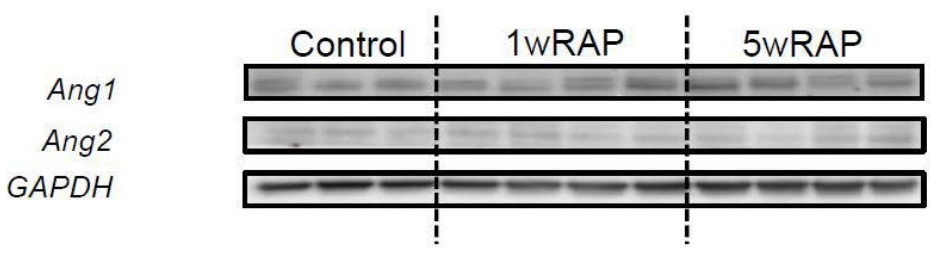

B

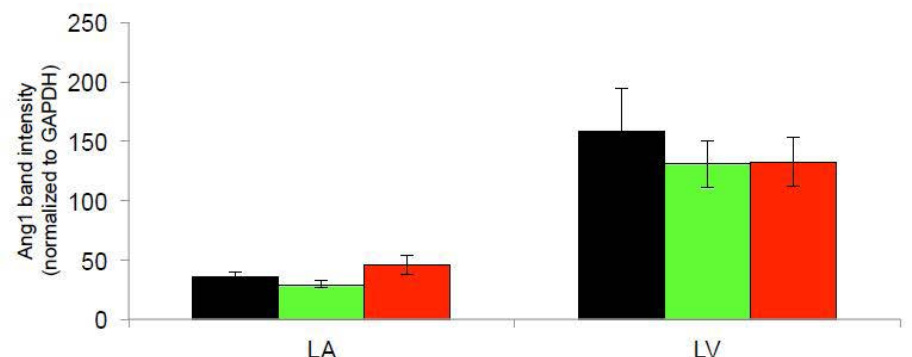

C

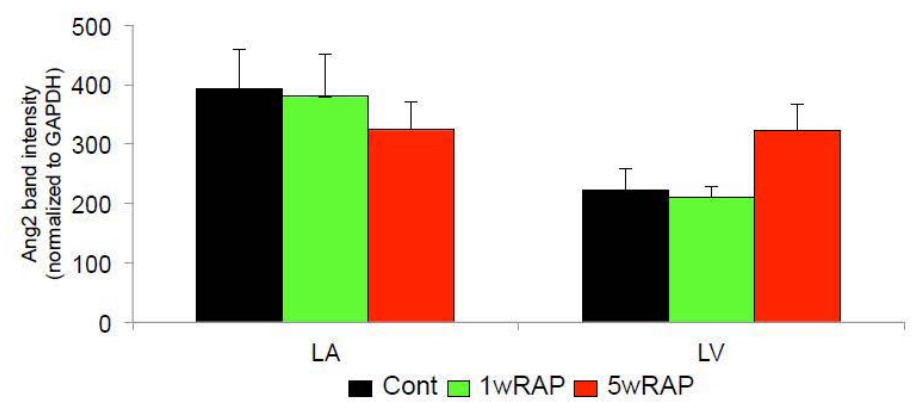

Figure 8. Quantification of Ang1 and Ang2 protein levels.

(A) Western blots displayed similar levels of Ang1 in the LA of 1WRAP $(n=7)$ and $5 W R A P(n=8)$ compared to control ( $n=7)$. (B) Quantified band intensity (normalized to GAPDH content) in the LA and LV for (B) ANg1 and (C) Ang2. Values are expressed as mean \pm SEM.

\section{DISCUSSION}

In this study we have studied hypoxic and angiogenic signalling and vascular remodeling in pigs at two relatively early time points in the pathogenesis of AF. Atrial ischemia may be an important factor in the vicious cycle that leads to progression of AF. On the one hand, ischemia can create a substrate for AF. In a dog model, ligation of an atrial artery, both acutely ${ }^{14}$ and chronically ${ }^{15}$ increases AF stability. In humans, ischemic heart disease is a risk factor for $\mathrm{AF}^{16}$. Also, acute myocardial infarction is complicated by new onset AF in 5 to $15 \%$ of patients, ${ }^{17}$ particularly when atrial branches are affected ${ }^{18,19}$

Conversely, AF also leads to atrial ischemia. We have previously shown that acute AF leads to acute supply/demand ischemia, as evidenced by lactate production within minutes of $\mathrm{AF}^{7}$, and reactive hyperemia following a short period of $\mathrm{AF}^{8}$. Atrial 
ischemia during prolonged $\mathrm{AF}$ is indicated by a decrease in phosphocreatine and an increase in HIF1a in the first weeks of AF in the goat model of AF. Increased atrial levels of HIF1a have also been reported in AF patients. The phenomenon of atrial myocyte hibernation ${ }^{20}$ which is similar to ventricular remodeling during low-flow ischemia, may be viewed as an adaptation of energy shortage/ ischemia.

Under normoxic conditions, HIF1a is continuously produced, but rapidly degraded upon hydroxylation of proline residues. During hypoxia, prolyl hydroxylation is inhibited, leading to an accumulation of HIF1a ${ }^{21}$. HIF1a can then combine with the constitutively present HIF1 $\beta$ to form a dimer that alters the expression of several genes. By increasing expression of CTGF, it can lead to interstitial fibrosis, at least in the kidney ${ }^{22,23}$. In pigs with AF maintained by RAP, we observed increased levels of HIF1a in both the LA and LV after 1 week, indicating cardiac oxygen shortage as a result of AF. After 5 weeks, HIF1a levels had normalized in the LV, but were still elevated in the LA. The sustained hypoxic signaling in the LA was associated with increased expression of VEGF after 5 weeks. Importantly, the HIF1a -HIF12 dimer also activates transcription of VEGF ${ }^{21}, 24$. VEGF is the pivotal cytokine leading to angiogenesis (formation of new capillaries) and, to a lesser degree, arteriogenesis (formation of new arterioles) ${ }^{25-27}$. In our pig model of AF, we did not observe any change in the number and size of arterioles, at either 1 or 5 weeks of RAP, in either the LA or LV. The number of capillaries was not affected in the LV, but showed a pronounced decrease in the LA in the 5WRAP group. The size distribution displayed a shift to smaller capillary diameters after 1 and 5 weeks of AF in the LA and shift to larger diameters after 5 weeks of AF in the LV. The shift towards smaller size in the LA may reflect a process of capillary sprouting as part of ongoing angiogenesis ${ }^{28}$. However, the number of capillaries in the LA was decreased after 5 weeks of AF. The loss of atrial capillaries will increase oxygen diffusion distances and therefore negatively affect the supply/ demand balance. In general, it will decrease the capacity of the atrial myocardium to respond to fluctuation in oxygen demand.

Normally, interaction with pericytes is required to stabilize (newly formed) capillaries, and a loss of capillaries (rarefaction) occurs when pericytes detach from capillaries ${ }^{29}$. In several organ systems, most notably in the kidney, the interaction between pericytes and endothelial cells is affected by the balance between angiopoietin-1 and $-2^{30-32}$, This interaction is stabilized by angiopoietin-1 but destabilized by angiopoietin-2. In the pig LA and LV, we did not observe a change in angiopoietin-1 and -2 expression as a result of RAP. Therefore, the capillary rarefaction we have observed cannot be explained by the shift in the balance between angiopoietins. Capillary rarefaction is often associated with progressive fibrosis e.g. in the hypoxic kidney ${ }^{30}$ and in the ventricles of heart failure patients with preserved ejection fraction ${ }^{33}$.

One of the reasons for this association is that, after detaching from capillaries, 
pericytes can transdifferentiate into fibroblasts that increase (interstitial) fibrosis ${ }^{29}$. In the goat model of AF, we have shown that prolonged AF is indeed hallmarked by interstitial/ endomysial fibrosis, that gradually develops over a time course of months and contributes to the progression of $\mathrm{AF}^{6,34}$. In this way, capillary rarefaction may reflect an important mechanism leading to the formation of a structural substrate for AF. 


\section{REFERENCES}

1. Wyndham CR. Atrial fibrillation: the most common arrhythmia. Texas Heart Institute journal / from the Texas Heart Institute of St Luke's Episcopal Hospital, Texas Children's Hospital 2000;27:257-267.

2. Schotten U, Verheule S, Kirchhof P, Goette A. Pathophysiological mechanisms of atrial fibrillation: a translational appraisal. Physiological reviews 2011;91:265-325.

3. Wijffels MC, Kirchhof CJ, Dorland R, Allessie MA. Atrial fibrillation begets atrial fibrillation. A study in awake chronically instrumented goats. Circulation 1995;92:1954-1968.

4. Verheule S, Tuyls E, van Hunnik A, Kuiper M, Schotten U, Allessie M. Fibrillatory conduction in the atrial free walls of goats in persistent and permanent atrial fibrillation. Circulation Arrhythmia and electrophysiology 2010;3:590-599.

5. Ausma J, Wijffels M, Thone F, Wouters L, Allessie M, Borgers M. Structural changes of atrial myocardium due to sustained atrial fibrillation in the goat. Circulation 1997;96:3157-3163.

6. Verheule S, Tuyls E, Gharaviri A, Hulsmans S, van Hunnik A, Kuiper M, Serroyen J, Zeemering $\mathrm{S}$, Kuijpers NH, Schotten U. Loss of continuity in the thin epicardial layer because of endomysial fibrosis increases the complexity of atrial fibrillatory conduction. Circulation Arrhythmia and electrophysiology 2013;6:202-211.

7. van Bragt KA, Nasrallah HM, Kuiper M, Luiken JJ, Schotten U, Verheule S. Atrial supplydemand balance in healthy adult pigs: coronary blood flow, oxygen extraction, and lactate production during acute atrial fibrillation. Cardiovascular research 2014;101:9-19.

8. van Bragt KA, Nasrallah HM, Kuiper M, van Hunnik A, Kuijpers NH, Schotten U, Verheule S. Dynamic regulation of atrial coronary blood flow in healthy adult pigs. Heart rhythm : the official journal of the Heart Rhythm Society 2015;12:991-1000.

9. Thijssen VL, van der Velden HM, van Ankeren EP, Ausma J, Allessie MA, Borgers M, van Eys $\mathrm{GJ}$, Jongsma HJ. Analysis of altered gene expression during sustained atrial fibrillation in the goat. Cardiovascular research 2002;54:427-437.

10. Gramley F, Lorenzen J, Jedamzik B, Gatter K, Koellensperger E, Munzel T, Pezzella F. Atrial fibrillation is associated with cardiac hypoxia. Cardiovascular pathology : the official journal of the Society for Cardiovascular Pathology 2010;19:102-111.

11. Ausma J, Coumans WA, Duimel H, Van der Vusse GJ, Allessie MA, Borgers M. Atrial high energy phosphate content and mitochondrial enzyme activity during chronic atrial fibrillation. Cardiovascular research 2000;47:788-796.

12. Rahimtoola SH. From coronary artery disease to heart failure: role of the hibernating myocardium. The American journal of cardiology 1995;75:16E-22E.

13. Dedkov El, Christensen LP, Weiss RM, Tomanek RJ. Reduction of heart rate by chronic beta1- 
adrenoceptor blockade promotes growth of arterioles and preserves coronary perfusion reserve in postinfarcted heart. American journal of physiology Heart and circulatory physiology 2005;288:H2684-2693.

14. Sinno H, Derakhchan K, Libersan D, Merhi Y, Leung TK, Nattel S. Atrial ischemia promotes atrial fibrillation in dogs. Circulation 2003;107:1930-1936.

15. Nishida K, Qi XY, Wakili R, Comtois P, Chartier D, Harada M, Iwasaki YK, Romeo P, Maguy A, Dobrev D, Michael G, Talajic M, Nattel S. Mechanisms of atrial tachyarrhythmias associated with coronary artery occlusion in a chronic canine model. Circulation 2011;123:137-146.

16. Krahn AD, Manfreda J, Tate RB, Mathewson FA, Cuddy TE. The natural history of atrial fibrillation: incidence, risk factors, and prognosis in the Manitoba Follow-Up Study. The American journal of medicine 1995;98:476-484.

17. Goldberg RJ, Yarzebski J, Lessard D, Wu J, Gore JM. Recent trends in the incidence rates of and death rates from atrial fibrillation complicating initial acute myocardial infarction: a community-wide perspective. American heart journal 2002;143:519-527.

18. Kyriakidis M, Barbetseas J, Antonopoulos A, Skouros C, Tentolouris C, Toutouzas P. Early atrial arrhythmias in acute myocardial infarction. Role of the sinus node artery. Chest 1992;101:944-947.

19. Hod H, Lew AS, Keltai M, Cercek B, Geft IL, Shah PK, Ganz W. Early atrial fibrillation during evolving myocardial infarction: a consequence of impaired left atrial perfusion. Circulation 1987;75:146-150.

20. Ausma J, Litjens N, Lenders MH, Duimel H, Mast F, Wouters L, Ramaekers F, Allessie M, Borgers $\mathrm{M}$. Time course of atrial fibrillation-induced cellular structural remodeling in atria of the goat. Journal of molecular and cellular cardiology 2001;33:2083-2094.

21. Semenza GL. Angiogenesis in ischemic and neoplastic disorders. Annual review of medicine 2003;54:17-28.

22. Higgins DF, Biju MP, Akai Y, Wutz A, Johnson RS, Haase VH. Hypoxic induction of Ctgf is directly mediated by Hif-1. American journal of physiology Renal physiology 2004;287:F12231232.

23. Kimura K, Iwano M, Higgins DF, Yamaguchi Y, Nakatani K, Harada K, Kubo A, Akai Y, Rankin EB, Neilson EG, Haase VH, Saito Y. Stable expression of HIF-1alpha in tubular epithelial cells promotes interstitial fibrosis. American journal of physiology Renal physiology 2008;295:F1023-1029.

24. Lee SH, Wolf PL, Escudero R, Deutsch R, Jamieson SW, Thistlethwaite PA. Early expression of angiogenesis factors in acute myocardial ischemia and infarction. The New England journal of medicine 2000;342:626-633.

25. Nagy JA, Dvorak AM, Dvorak HF. VEGF-A(164/165) and PIGF: roles in angiogenesis and 
arteriogenesis. Trends in cardiovascular medicine 2003;13:169-175.

26. Buschmann I, Schaper W. Arteriogenesis Versus Angiogenesis: Two Mechanisms of Vessel Growth. News in physiological sciences : an international journal of physiology produced jointly by the International Union of Physiological Sciences and the American Physiological Society 1999;14:121-125.

27. Heil M, Eitenmuller I, Schmitz-Rixen T, Schaper W. Arteriogenesis versus angiogenesis: similarities and differences. Journal of cellular and molecular medicine 2006;10:45-55.

28. Egginton S, Hudlicka O. Early changes in performance, blood flow and capillary fine structure in rat fast muscles induced by electrical stimulation. The Journal of physiology 1999;515 ( Pt 1):265-275.

29. Schrimpf C, Teebken OE, Wilhelmi M, Duffield JS. The role of pericyte detachment in vascular rarefaction. Journal of vascular research 2014;51:247-258.

30. Khairoun M, van der Pol P, de Vries DK, Lievers E, Schlagwein N, de Boer HC, Bajema IM, Rotmans JI, van Zonneveld AJ, Rabelink TJ, van Kooten C, Reinders ME. Renal ischemiareperfusion induces a dysbalance of angiopoietins, accompanied by proliferation of pericytes and fibrosis. American journal of physiology Renal physiology 2013;305:F901-910.

31. Tanaka T, Nangaku M. Angiogenesis and hypoxia in the kidney. Nature reviews Nephrology 2013;9:211-222.

32. Gonzalez-Quesada C, Cavalera M, Biernacka A, Kong P, Lee DW, Saxena A, Frunza O, Dobaczewski M, Shinde A, Frangogiannis NG. Thrombospondin-1 induction in the diabetic myocardium stabilizes the cardiac matrix in addition to promoting vascular rarefaction through angiopoietin-2 upregulation. Circulation research 2013;113:1331-1344.

33. Mohammed SF, Hussain S, Mirzoyev SA, Edwards WD, Maleszewski JJ, Redfield MM. Coronary microvascular rarefaction and myocardial fibrosis in heart failure with preserved ejection fraction. Circulation 2015;131:550-559.

34. Ausma J, van der Velden HM, Lenders MH, van Ankeren EP, Jongsma HJ, Ramaekers FC, Borgers M, Allessie MA. Reverse structural and gap-junctional remodeling after prolonged atrial fibrillation in the goat. Circulation 2003;107:2051-2058. 



\section{Atrial fibrillation alters the behavior of atrial and ventricular small muscular arteries}

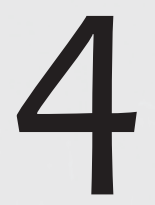

Hussein M Nasrallah; Paul M. Schiffers;

Dragan Opacic; Marion Kuiper; Charalambos Antoniades; Ulrich Schotten; Sander Verheule.

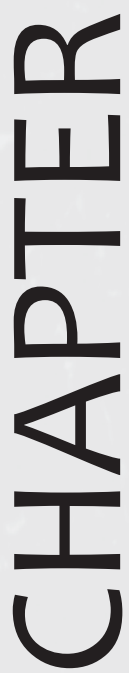




\section{ABSTRACT}

Background: We have previously shown acute AF leads to acute supply/demand ischemia in the atrial myocardium. To investigate the ensuing alterations in the coronary vasculature we have studied function and structure of left atrial (LA) and left ventricular (LV) small muscular arteries (SMA) in healthy control pigs and pigs with one week of rapid atrial pacing (RAP).

Methods: SMA were isolated from the LA and LV of 7 RAP and 8 control pigs and mounted to record vascular wall tension by wire myography. In addition to stiffness and receptor-independent vasoconstriction $(62.5 \mathrm{mM} \mathrm{KCl})$, dose-response curves were determined for U46619 (a stable thromboxane A2 analogue), and endothelin-1 (ET-1), representing receptor-mediated vasoconstriction; bradykinin (BK, endothelium-dependent vasodilation) and sodium nitroprusside (SNP, endotheliumindependent vasodilation).

Results: After one week of RAP, both LA and LV SMA displayed a significantly higher contractility in response to $\mathrm{KCL}$ compared to control $(5.3 \pm 0.6 \mathrm{vs} .3 .5 \pm 0.5 \mathrm{mN} / \mathrm{mm}$ for the LA and $4.8 \pm 0.4$ vs. $2.4 \pm 0.5 \mathrm{mN} / \mathrm{mm}$ for the LV; $P<0.05)$. Furthermore, LA and LV SMA from the RAP group produced a higher vascular wall tension in response to the highest dose of U46619. The response to ET-1 did not differ significantly between the groups in either LA and LV SMA. The LA SMA from RAP group displayed an higher sensitivity to $\mathrm{BK}$, with an $\mathrm{EC}_{50}$ in control and RAP animals of $1.3 \times 10^{-09} \pm 2.6 \times 10^{-10} \mathrm{M}$ and $3.0 \times 10^{-10} \pm 6.1 \times 10^{-11} \mathrm{M}(\mathrm{p}<0.01)$. The response to SNP was similar between the groups both for the LA and LV. These changes in vascular pharmacology were not associated with smooth muscle cell hypertrophy.

Conclusions: In a relatively early stage of AF pathogenesis, sensitivity of LA and LV SMA to both vasoconstrictors and dilators is enhanced. The increased vascular responsiveness may represent an adaptive reaction to the higher myocardial oxygen demand during AF. 


\section{INTRODUCTION}

Atrial fibrillation (AF) is the most common sustained cardiac tachyarrhythmia. The high activation rates during AF are likely to lead to an increase in atrial energy and oxygen demand. If the increased demand is not met by an increase in the oxygen supply, a state of supply/demand ischemia will ensue. Indeed, we have recently shown that acute AF leads to acute supply/ demand ischemia, as evidenced by atrial lactate production ${ }^{1}$ and a phase of reactive hyperemia following a short period of $\mathrm{AF}^{2}$.

This state of supply/ demand ischemia may persist for a prolonged period. In RA biopsies from patients with permanent AF, markers of hypoxia (HIF1a) and angiogenesis (VEGF) were elevated ${ }^{3}$. In a goat model, AF leads to a reduction in atrial levels of creatine phosphate ${ }^{4}$ and an increase in HIF1a in the first weeks ${ }^{5}$. To our knowledge, no previous reports exist on the effect of AF on the behavior of the small muscular arteries (SMA; compliance and resistance arteries) that are the main determinants of coronary blood flow regulation.

In the present study, we investigated the effect of AF on vascular responsiveness at a relatively early time point of its pathogenesis (one week of rapid atrial pacing). We have studied isolated SMA from the LA and LV to assess receptor-independent $(\mathrm{KCl})$ and receptor-dependent (U46619 and ET-1) vasoconstriction and to endotheliumdependent (BK) and endothelium-independent (SNP) vasodilation. In addition, stiffness and smooth muscle hypertrophy were studied. 


\section{METHODS}

Animal model. Two groups of adult landrace pigs were studied. One group of pigs served as healthy controls ( $n=9,40-55 \mathrm{~kg})$ In the RAP group, $(n=7,50-55 \mathrm{~kg})$ pigs received a right atrial endocardial pacemaker lead (Capsurefix 5568, Medtronic Inc. Minneapolis, Minnesota) connected to an implantable subcutaneous pacemaker (Itrel II, Medtronic, Minneapolis, MN). All animal procedures were in agreement with international standards of research animal use and were approved by the local ethical committee for animal experiments of Maastricht University.

After one week of recovery after pacemaker implantation, pigs were sedated with an intramuscular injection of Zoletil $(5-8 \mathrm{mg} / \mathrm{kg})$. An ECG was performed to confirm atrial capture, and rapid atrial pacing at (60obpm) with four times pacing threshold was initiated.

Tissue harvesting and vessel isolation. Both RAP pigs (after 1 week of pacing) and control pigs were sedated with Zoletil ( $5-8 \mathrm{mg} / \mathrm{kg}$ I.M.). In the RAP group, pacing was interrupted during the induction of anesthesia. Anesthesia was maintained with fentanyl $(6 \mathrm{mg} / \mathrm{kg} / \mathrm{h})$, midazolam $(0.8 \mathrm{mg} / \mathrm{kg} / \mathrm{h})$ and propofol $(0.3 \mathrm{mg} / \mathrm{kg} / \mathrm{h})$. A left sided thoracotomy was performed to expose the heart and after 30 minutes of stabilization the heart was excised and a strip of tissue around the left circumflex artery, with a $1-2 \mathrm{~cm}$ width of the LA and LV myocardium attached was collected and stored in a $4^{\circ} \mathrm{C}$ HEPES solution. Starting dissection from the LCX, SMA were isolated from the tissue using a microscopic setup and stored in HEPES physiological buffer salt solution that contained in (mM): $\mathrm{NaCl} 144, \mathrm{KCl} 4.7, \mathrm{CaCl}_{2} 2.5, \mathrm{MgSO}_{4} 1.2, \mathrm{KH}_{2} \mathrm{PO}_{4}$ 1.2, HEPES 14.9 and glucose 5.5 with a $\mathrm{pH}$ set to 7.4 .

Vasomotor studies. Isolated arterial segments of approximately $2 \mathrm{~mm}$ in length were mounted in multi-channel wire myograph for recording isometric force development (Multi-channel wire myograph system, DMT, Denmark) as described previously ${ }^{6}$. Arteries where then equilibrated for 60 minutes at $37^{\circ} \mathrm{C}$ in an oxygenated Krebs-Ringer bicarbonate buffer (KRB) containing in ( $\mathrm{mM}$ ): NaCL: 118.5; $\mathrm{KCL}: 4.7 ; \mathrm{CaCl}_{2}: 2.5 ; \mathrm{KH}_{2} \mathrm{PO}_{4}: 1.2 ; \mathrm{NaHCO}_{3}: 25.0$ and glucose: 5.5. After equilibration, a standardized normalization procedure 67 was performed to determine the lumen diameter $\left(\mathrm{d}_{100}\right)$ the artery would have had in vivo under a transmural pressure of $100 \mathrm{mmHg}$. Arteries where then set to the lumen diameter $d_{1}=0.9 * d_{100}$ for optimal active force development. Subsequently, the vessels were left to equilibrate for 3omins. Viability and contractile performance of vessels was tested by addition of 62.5 $\mathrm{mM} \mathrm{KCl}$ (receptor-independent vasoconstriction). Vessels that did not produce any vascular wall tension in response to $\mathrm{KCL}$ were excluded. Subsequently, a doseresponse curve was recorded for $\mathrm{U} 46619\left(10^{-9} \mathrm{M}\right.$ to $\left.10^{-5.5} \mathrm{M}\right)$. In the presence of $10^{-5.5} \mathrm{M}$ U46619 as a precontraction, dose-response curves were recorded for bradykinin 
(BK, $10^{-11} \mathrm{M}$ to $10^{-7} \mathrm{M}$, endothelium-dependent vasodilation) and the $\mathrm{NO}$ donor Sodium Nitroprusside (SNP, $10^{-8} \mathrm{M}$ to $10^{-4.5} \mathrm{M}$, endothelium-independent vasodilation). Finally, a dose-response curve for endothelin-1 mediated vasoconstriction ( $10^{-10} \mathrm{M}$ to $10^{-6.5} \mathrm{M}$ ) was recorded.

Vascular Morphometery. After vasomotor studies, vessels were fixated with 4\% buffered formalin, embedded in paraffin, sliced in $5 \mu \mathrm{m}$ sections. Vessels were stained with Hematoxylin and Eosin (H\&E) to determine the density of smooth muscle cells or with Lawson staining to determine the medial cross-sectional area (mCSA, the area in between internal and elastic lamina). Sections were photographed at 200x magnification and analyzed using Image J software (version 1.46r, National Institute of Health, USA) in a blinded fashion. Quantification of smooth muscle cell density was performed by counting the nuclei of smooth muscle cells and normalizing this number to the smooth muscle area, using ImageJ.

Western Blot. After excision of the heart, tissue samples from the LA were snapfrozen in liquid nitrogen and stored at $-80^{\circ} \mathrm{C}$ for Western blotting. Proteins were separated by SDS-PAGE on 4-12\% Bis-Tris Standard XT precast gels (Bio-Rad) and moved to nitrocellulose membranes. The membranes were probed with primary antibody for eNOS (BD Biosciences 61029) in 5\% bovine serum albumin. The protein bands were visualized using enhanced chemiluminescence, and the immunoblot intensities were quantified using Bio-Rad Quantity One (Version 4.6.6, Bio-Rad laboratories Inc., USA)

Chemicals. U46619 (Cayman Chemical \#16450) was dissolved in 100\% ethanol. Bradykinin acetate salt (Sigma-Aldrich B3259) and Endothelin-1 (Sigma-Aldrich E7764) were dissolved in distilled water.

Statistical Analysis. Concentration response curves were fitted to a non-linear sigmoid regression curve (Graph-pad Prism 5.0). Results are expressed as mean $\pm S E M$. Statistical analysis comparing the highest effect size $\left(\mathrm{E}_{\max }\right)$ and the $\mathrm{EC}_{50}$ was performed using a mixed model with Bonferroni correction. A p value less than 0.05 was considered to be statistically significant.

\section{RESULTS}

\section{Passive properties and receptor-independent vasoconstriction}

The optimal lumen diameter $\left(0.9 * \mathrm{~d}_{100}\right)$ of vessels did not differ significantly between the control and RAP group (figure $1 \mathrm{~A}$ ). Passive wall tension was plotted against diameter, and the slope of the curve was calculated to determine stiffness. Vascular stiffness did not differ significantly between the groups in either the LA or LV 
(figure 1B). Then, $62.5 \mathrm{mM} \mathrm{KCL}$ was administrated to assess receptor-independent vasoconstriction. Both the LA and LV arteries from RAP animals generated a significantly larger vascular wall tension compared to control (figure 1C).

A

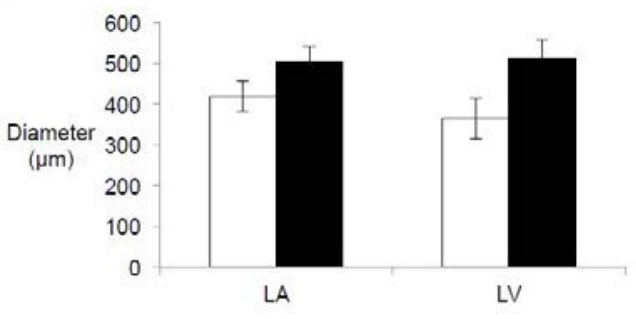

C

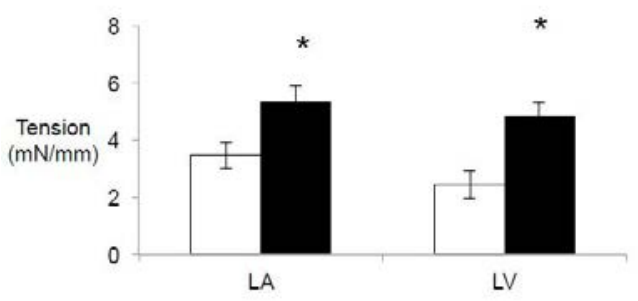

B

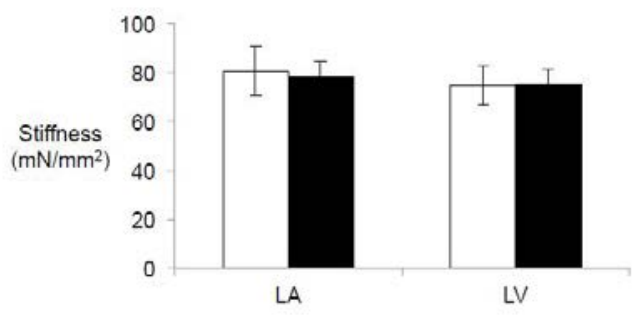

D
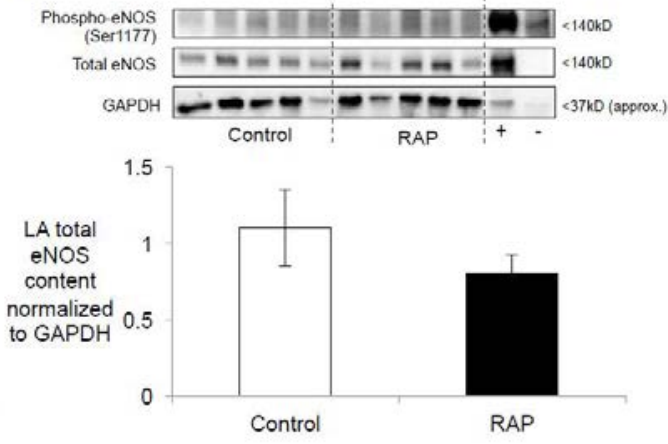

RAP

Figure 1. Passive properties and eNOS content of SMA.

(A) Optimal lumen diameter of SMA in LA control $(n=7)$ and RAP $(n=7)$ and LV control $(n=8)$ and RAP $(n=6)(B)$ stiffness determined by stepwise, progressive stretching in LA control $(n=7)$ and RAP $(n=7)$ and LV control $(n=8)$ and RAP $(n=6)(C)$ Vascular wall tension in response to $62.5 \mathrm{mM} \mathrm{KCL}$ of SMA in LA control $(n=7)$ and RAP $(n=7)$ and LV control $(n=9)$ and RAP $(n=6) . * P<0.05$ control vs. RAP (D) illustrative western blots and bar graph depicting the LA total eNOS content in control $(n=6)$ and RAP $(n=6)$ pigs.

\section{Receptor-dependent vasoconstriction}

Both for the LA and LV, the wall tension in response to the highest concentration of U46619 was significantly higher in RAP SMA compared to control (figure 2A\&C, table 1). When normalized to the receptor-independent vasoconstriction $(\mathrm{KCl})$, the maximal response to $\mathrm{U}_{46619} \mathrm{E}_{\max }$ showed a similar, but non-significant trend towards increased maximal tension in the RAP group (figure $2 B \& D$, table 1). The concentration with the half-maximal effect $\left(\mathrm{EC}_{50}\right)$ was not different between control and RAP SMA (figure 2, table 1).

In addition, ET-1 mediated vasoconstriction was assessed. The dose response curve 
of ET-1 showed a non-significant trend towards an increased maximal effect in the LA and a significant increase in the LV of RAP SMA compared to control (figure 3A\&C, table 1). However, the difference between the control and RAP SMA was not present when the tension was normalized to the receptor-independent vasoconstriction $(K C L)$ as displayed in (figure $3 B \& D$, table 1). RAP did not affect the $\mathrm{EC}_{50}$ in either $L A$ or LV (table 1).

Table 1. Values for highest dose concentration and concentrations with half-maximal effect (EC50)

\begin{tabular}{|c|c|c|c|c|}
\hline $\mathrm{EC}_{50}(\mathrm{M})$ & & control & RAP & p-value \\
\hline \multirow[t]{2}{*}{ U46619 } & LA & $3.2 \mathrm{E}-07 \pm 1.2 \mathrm{E}-07$ & $8.0 \mathrm{E}-08 \pm 3.2 \mathrm{E}-08$ & 0.19 \\
\hline & LV & $2.0 \mathrm{E}-07 \pm 1.2 \mathrm{E}-07$ & 1.1E- $07 \pm 3.7 \mathrm{E}-08$ & 0.28 \\
\hline \multirow[t]{2}{*}{ ET-1 } & LA & $1.0 \mathrm{E}-07 \pm 1.4 \mathrm{E}-08$ & $8.0 \mathrm{E}-08 \pm 9.4 \mathrm{E}-09$ & 0.21 \\
\hline & LV & $8.2 E-08 \pm 1.5 E-08$ & $1.2 \mathrm{E}-07 \pm 2.0 \mathrm{E}-08$ & 0.37 \\
\hline \multirow[t]{2}{*}{ BK } & LA & 1. $3 \mathrm{E}-09 \pm 2.6 \mathrm{E}-10$ & $3.0 \mathrm{E}-10 \pm 6.1 \mathrm{E}-11$ & 0.01 \\
\hline & LV & 1.7E-09 $\pm 1.0 \mathrm{E}-09$ & $4.4 \mathrm{E}-10 \pm 1.3 \mathrm{E}-10$ & 0.26 \\
\hline \multirow[t]{2}{*}{ SNP } & LA & $2.1 \mathrm{E}-06 \pm 6.3 \mathrm{E}-07$ & $2.8 \mathrm{E}-06 \pm 8.2 \mathrm{E}-07$ & 0.52 \\
\hline & LV & 4.1E-06 $\pm 2.7 \mathrm{E}-06$ & $2.8 \mathrm{E}-06 \pm 7.9 \mathrm{E}-07$ & 0.28 \\
\hline$E_{\max }$ & & control & RAP & p-value \\
\hline \multirow{2}{*}{$\begin{array}{l}10^{-5.5} \cup 46619(\% \\
K C L)\end{array}$} & LA & $54.6 \pm 10.1$ & $76.7 \pm 8.7$ & 0.28 \\
\hline & LV & $64.1 \pm 9.0$ & $64.7 \pm 9.5$ & 0.96 \\
\hline \multirow{2}{*}{$\begin{array}{l}10^{-5.5} \mathrm{U} 46619 \\
(\mathrm{mN} / \mathrm{mm})\end{array}$} & LA & $1.9 \pm 0.5$ & $3.8 \pm 0.5$ & 0.04 \\
\hline & LV & $1.1 \pm 0.2$ & $3.5 \pm 0.5$ & 0.001 \\
\hline \multirow{2}{*}{$\begin{array}{l}10^{-6.5} \mathrm{ET}-1 \\
(\% \mathrm{KCL})\end{array}$} & LA & $113.2 \pm 20.3$ & $105.6 \pm 2.7$ & 0.96 \\
\hline & LV & $125.4 \pm 17.5$ & $137 \pm 29.3$ & 0.86 \\
\hline \multirow{2}{*}{$\begin{array}{l}10^{-6.5} \text { ET-1 } \\
(\mathrm{mN} / \mathrm{mm})\end{array}$} & LA & $4.2 \pm 0.8$ & $5.7 \pm 0.8$ & 0.39 \\
\hline & LV & $2.2 \pm 0.4$ & $5.6 \pm 0.6$ & 0.042 \\
\hline \multirow{2}{*}{$\begin{array}{l}10^{-4.5} \text { SNP } \\
(\%)\end{array}$} & LA & $101.9 \pm 4.5$ & $103.3 \pm 8.5$ & 0.91 \\
\hline & LV & $87.3 \pm 11.2$ & $90.2 \pm 7.0$ & 0.66 \\
\hline \multirow{2}{*}{$\begin{array}{l}10^{-7} \mathrm{BK} \\
(\%)\end{array}$} & LA & $100.0 \pm 0.4$ & $101.6 \pm 1.4$ & 0.37 \\
\hline & LV & $82.5 \pm 9.6$ & $103.9 \pm 4.0$ & 0.53 \\
\hline
\end{tabular}

\section{Endothelium-dependent and -independent vasodilatation}

In the presence of U46619, which produces a state of maximal thromboxaneinduced vascular constriction, dose-response curves for bradykinin (BK) and sodium nitroprusside (SNP) were determined (endothelium-dependent and -independent relaxation, respectively). Both in the LA and LV, the dose-response for BK was shifted to the left in the RAP group with respect to the control group (figure 4A\&C). The increased sensitivity to $B K$ was confirmed by the significantly lower $\mathrm{EC}_{50}$ in SMA of the RAP group compared to control, in the LA.

The dose-response curve for the direct NO donor SNP did not show significant 


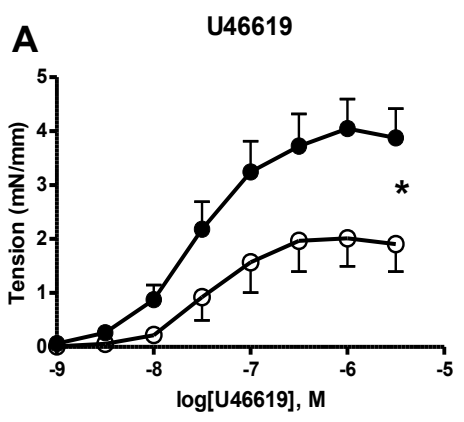

C

U46619

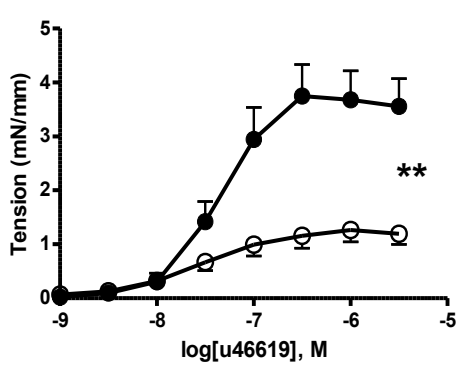

U46619

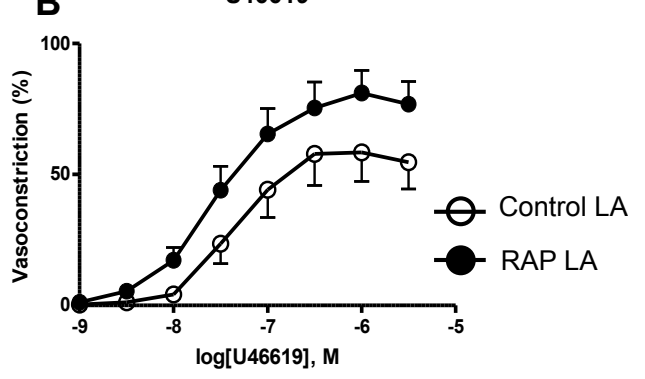

D

Figure 2. Receptor-dependent vasoconstriction.

Dose-response curves of $U 46619$ showing the vascular wall tension of $L A$ arteries in control $(n=7)$ and RAP pigs ( $n=7)(A)$ without and (B) with normalization to the receptor-independent vasoconstriction $(K C L)$. Dose-response curves of $U 46619$ showing the vascular wall tension of $L V$ arteries in control $(n=8)$ and RAP pigs $(n=6)$ (C) without and (D) with normalization to the receptor-independent vasoconstriction $(K C L) * * P<0.01$ control vs. RAP.

differences between the groups in either the LA or LV (figure 4B\&D). Correspondingly, the $\mathrm{EC}_{50}$ were similar between the control and RAP groups (table 1).

\section{Smooth muscle hypertrophy}

After vasomotor studies, all vessels were stored for histological analysis. Representative vascular sections with Lawson staining did not indicate major differences between control and RAP arteries (figure $5 A \& B$ ). Quantification of the medial cross-sectional area (mCSA) did not reveal significant differences between the control and RAP groups for either the LA or LV arteries (figure $5 \mathrm{E}$ ). This indicates that the smooth muscle cell layer did not undergo significant hypertrophy with 1 week of RAP. Furthermore, sections stained with Hematoxylin and Eosin (figure 5 B\&D) also did not display differences in the density of smooth muscle cells within the smooth muscle layer between groups for either LA or LV (figure $5 \mathrm{~F}$ ). 

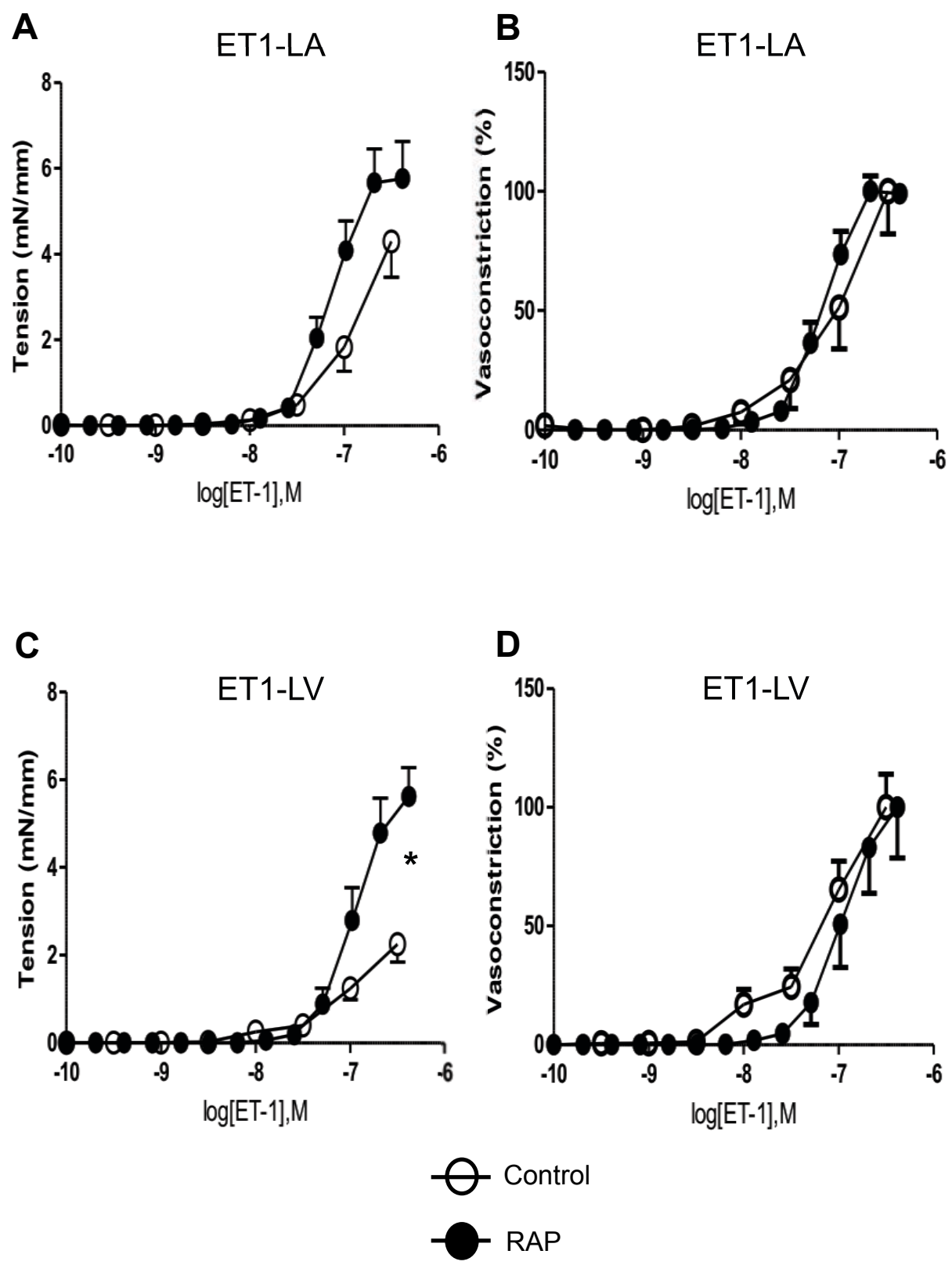

Figure 3. Endothelin-1 mediated vasoconstriction.

Dose-response curves of ET-1 showing the vascular wall tension of $L A$ arteries in control $(n=4)$ and RAP pigs $(n=5) \quad(A)$ without and (B) with normalization to the receptor-independent vasoconstriction $(K C L)$. Dose-response curves of ET-1 showing the vascular wall tension of $L V$ arteries in control $(n=6)$ and RAP pigs $(n=3)$ arteries (C) without and (D) with normalization to the receptor-independent vasoconstriction $(K C L) * * P<0.01$ control vs. RAP. 


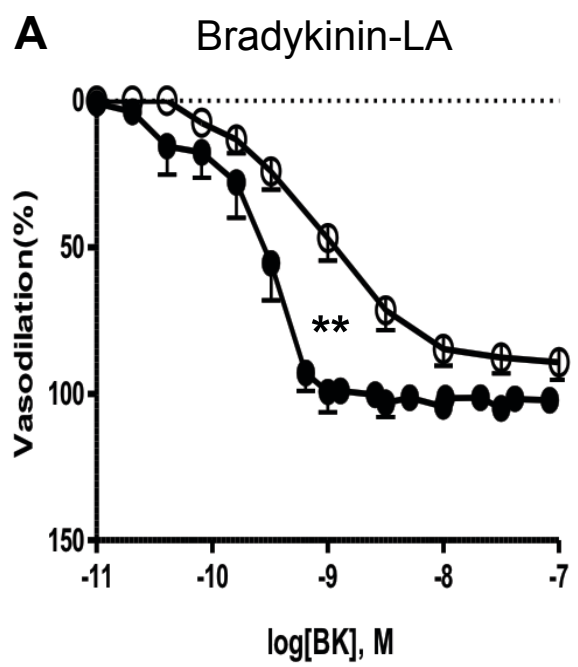

C

Bradykinin-LV
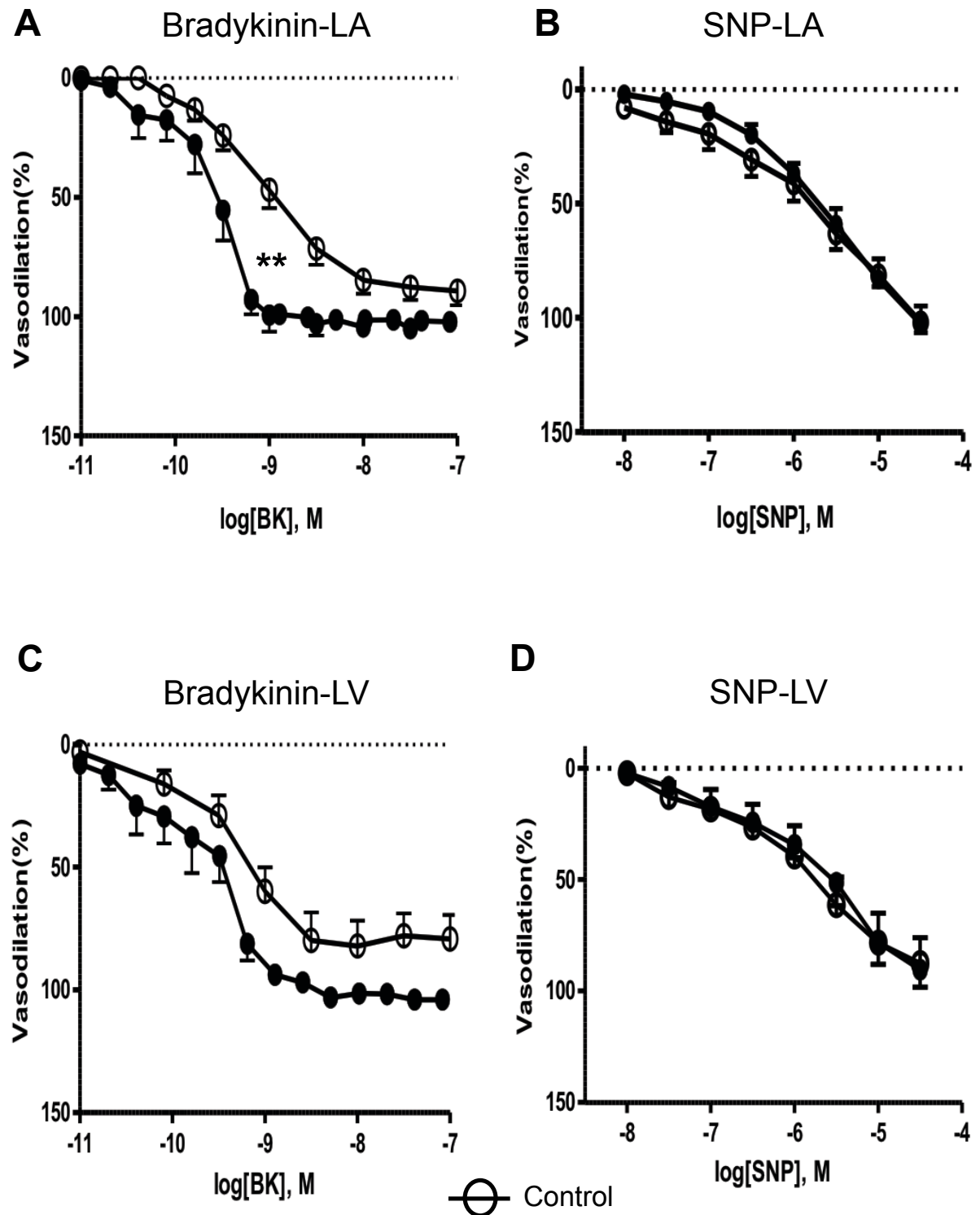

D
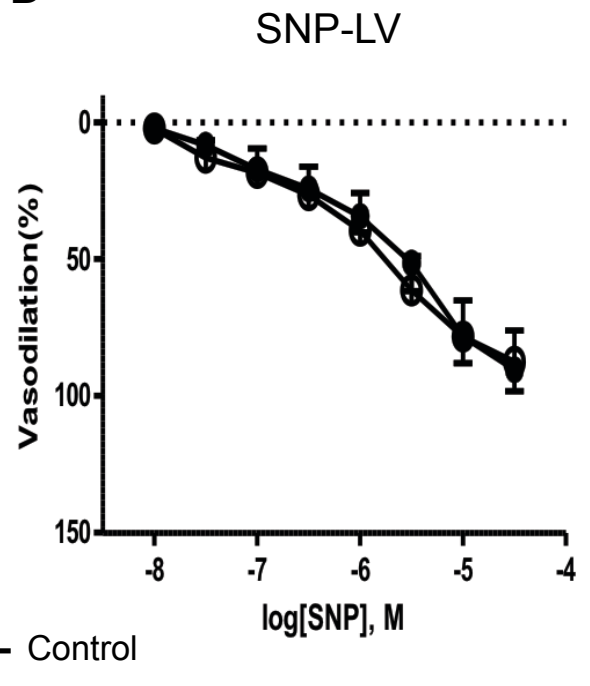

RAP

Figure 4. Endothelium-dependent and independent vasodilation.

Dose-response curves for LA arteries of (A) BK in control $(n=4)$ and RAP pigs $(n=4)$ and (B) SNP in control $(n=5)$ and RAP pigs $(n=7)$ mediated vasodilation. Dose-response curves for $L V$ arteries of $(A)$ BK in control $(n=3)$ and RAP pigs $(n=6)$ and $(B)$ SNP in control $(n=4)$ and RAP pigs $(n=6)$ mediated vasodilation. ${ }^{*} P<0.01$ control arteries vs. RAP arteries. 

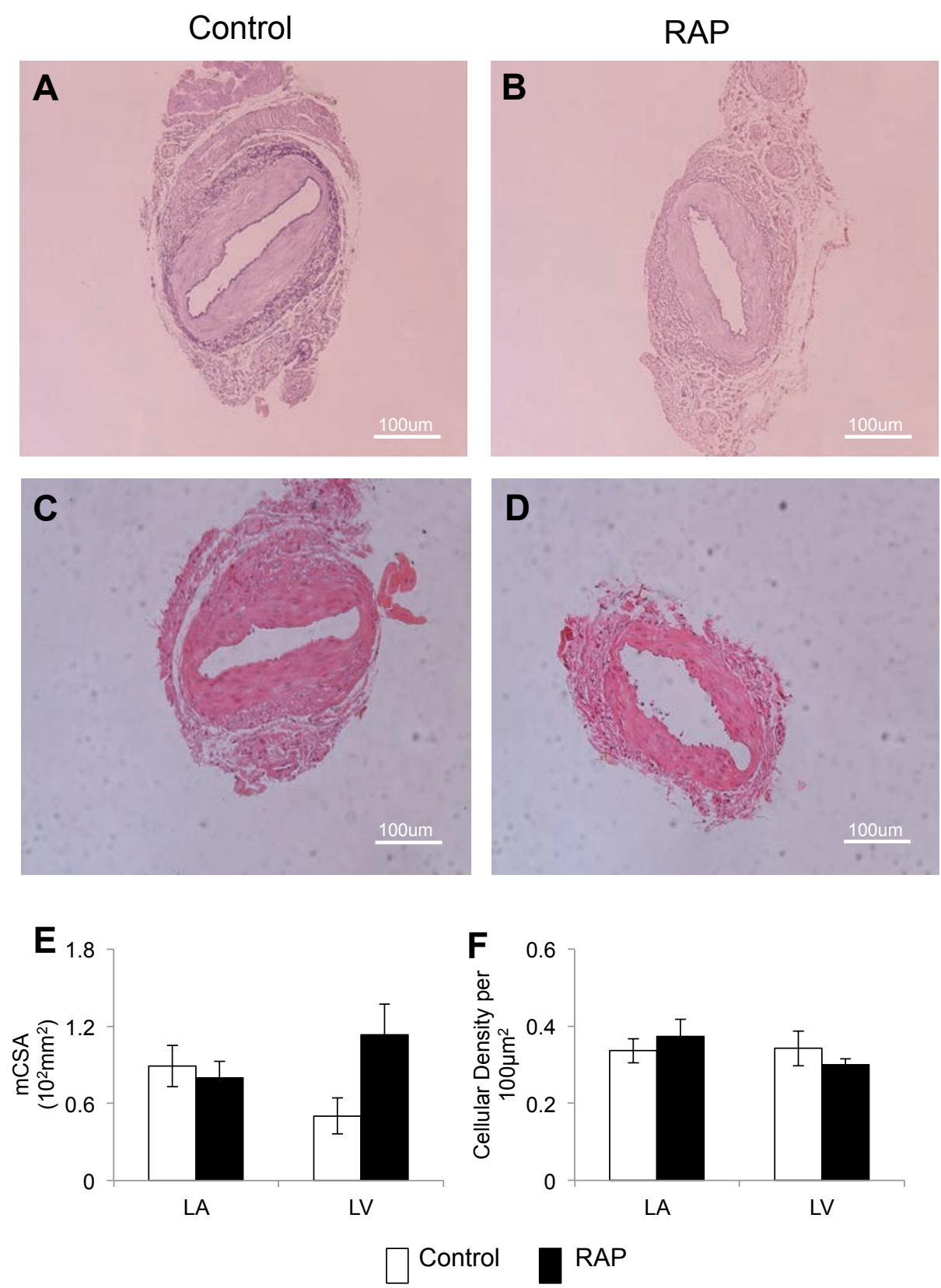

Figure 5. Absence of smooth muscle cell hypertrophy.

Lawson staining (used to determine the thickness of the smooth muscle layer thickness) in LA artery from (A) a control animal and (B) an RAP animal. Hematoxylin and Eosin staining (used to determine the smooth muscle cellular density) in LA artery from (A) a control animal and (B) an RAP animal. (E) Median cross-sectional area in LA and LV of SMA in control $(n=8)$ and RAP $(n=7)$ pigs and $(F)$ density of smooth muscle in LA and LV of SMA in control $(n=8)$ and RAP $(n=7)$ pigs which did not show significant differences between the groups. 


\section{DISCUSSION}

There is increasing evidence for oxygen shortage and energy depletion in fibrillating atria $^{1,2,4,5,8}$. Whether the supply/demand mismatch during AF is the result of altered vascular function in the atria has not been studied yet. The present study is the first to investigate the effect of AF on the vascular function of left atrial and ventricular coronary muscular arteries.

We show that 1 week of $A F$ in pigs leads increased vascular tension, in the absence of smooth muscle hypertrophy. Furthermore, the LA sensitivity to endothelium dependent vasodilation was enhanced, but this was not associated with an increase in eNOS tissue content.

\section{Vasoconstriction}

Depolarization of the SMC membrane with high extracellular $\mathrm{KCl}$ opens calcium channels, leading to a strong vasoconstriction. This 'receptor-independent' vasoconstriction was significantly larger in LA and LV SMA from the RAP compared to control. This could in principle be caused by SMC hypertrophy ${ }^{9},{ }^{10}$, but in our model, we did not observe SMC hypertrophy, as evidenced by similar medial cross section area and SMC density in control and RAP arteries. In addition, the stiffness of the isolated vessels was not affected by RAP meaning no smooth muscle structural alteration. An alternative explanation for the increased contractility involves SMC calcium handling. Bowles et al have demonstrated increased calcium current density in coronary smooth muscle of pigs after exercise training ${ }^{11}$. In that case, increased sympathetic activation during exercise was proposed as a stimulus for an increase in SMC calcium current density ${ }^{11}$, Several studies have reported increased sympathetic innervation in AF patients and animal models of AF ${ }^{12}$, 13, ${ }^{14}$, which may similarly contribute to increased expression of calcium channels in SMC. However, other aspects of SMC calcium handling may also be involved, e.g. increase in calcium mobilization and/ or calcium-sensitivity of the contractile apparatus. Our data do not allow us to distinguish between these possibilities.

ET- 1 is a vasoactive compound produced by coronary endothelial cells ${ }^{15}$. Autocrinally, ET-1 can promote vasodilation by binding to ET-B receptors localized on endothelial cells, stimulating production of the vasodilators $\mathrm{NO}$ and prostacyclin ${ }^{16}$. In the interstitial space, ET-1 binds to ET-A and ET-B receptors localized on smooth muscle cells, causing vasoconstriction ${ }^{17}$. Tissue levels of ET-1 are increased in the LA of patients with $\mathrm{AF}^{18}$. In a population of patients with $\mathrm{CHF}, \mathrm{AF}$ was also associated with increase plasma ET-1 ${ }^{19}$. Therefore, the effect of ET-1 on vascular function was studied. Although we did observe a larger contractility in response to ET-1 in RAP LA and LV vessels, this difference disappeared when the response was normalized to the receptor-independent vasoconstriction. This indicates that the contractile force, rather than the sensitivity to ET-1 was increased in the RAP group compared 
to control.

The thromboxane $\mathrm{A} 2$ analogue $\mathrm{U} 46619$ acts on thromboxane $\mathrm{A} 2$ receptors that are expressed by both endothelial and smooth muscle cells, resulting in vasoconstriction ${ }^{15}$. Thromboxane A2 is secreted during e.g. platelet aggregation ${ }^{15}$. Both LA and LV SMA from RAP animals displayed a substantial increase in vascular wall tension in response to U-46619 compared to arteries from control animals. After normalization to the receptor-independent vasoconstriction, a non-significant trend towards increased constriction remained in LA but not in the LV, indicating that the sensitivity to U46619 may be slightly enhanced in the SMA of RAP pigs.

As discussed above, there is no indication for SMC hypertrophy after 1 week of RAP. In isolated skeletal muscle arterioles, hypertension leads to an enhanced responsiveness to thromboxane $\mathrm{A} 2$ analogue and endothelin ${ }^{20}$, which was related to the increased calcium sensitivity of the contractile apparatus of the arteriolar SMCs ${ }^{20}$. In addition, aortic rings of diabetic rats have also displayed an increased vasoconstriction in response to ET-1 and U46619 ${ }^{21}$.

\section{Vasodilation}

In coronary arteries, BK stimulates the endothelial production of NO, prostaglandin $\mathrm{E}_{2}$ and endothelium-derived hyperpolarizing factor (EDHF), all of which can lead to relaxation of SMC 22. The phenomenon of 'endothelial dysfunction' is hallmarked by a decreased endothelial/ BK-dependent relaxation. However, in the RAP group, sensitivity to BK was higher than in control SMA only for the LA but not for the LV. The NO donor SNP can be applied to determine the sensitivity of the smooth muscle relaxation to NO directly ${ }^{23}$. We did not observe differences between the groups in the SNP dose-response curve for either the LA or LV SMA. Therefore, the increased LA sensitivity to $B K$ can be explained by an increased endothelial production of either $\mathrm{NO}$, prostaglandinE $\mathrm{E}_{2}$ or EDHF. Although we cannot distinguish these possibilities based on our data, a similar enhancement of bradykinin-dependent vasodilation has been observed in pig coronary resistance arterioles in response to exercise training ${ }^{24}$. There, both increased EDHF and NO production by nitric oxide synthase (NOS) were proposed as possible mechanisms for this enhanced endothelium-dependent vasodilation ${ }^{24}$. Another study has also indicated the beneficial effect of exercise in preserving endothelium-dependent relaxation in hypercholesterolemia pigs via nitric oxide synthase ${ }^{25}$. An improved endothelium-dependent relaxation was also observed in aortic rings from rats with small myocardial infarct ${ }^{26}$. Furthermore, in patients with stable coronary artery disease, physical exercise increased eNOS expression and eNOS phosphorylation, leading to improved endothelial function ${ }^{27}$. We did not observe changes in LA eNOS content as a result of AF. Therefore, the increased sensitivity to bradykinin in RAP arteries can not be attributed to increased levels of eNOS. However, our study reflects just one timepoint in the pathogenesis 
in AF. In an earlier study, we have demonstrated eNOS uncoupling in patients with persistent $A F$ and in goats after 6 months of $A F$, but not after 2 weeks of $A F^{28}$. In that context, it is conceivable that later stages of $\mathrm{AF}$ are hallmarked by endothelial dysfunction. The changes we have described here at the relatively early time point of 1 week of $\mathrm{AF}$ may represent an early, adaptive response to the higher oxygen demand during $\mathrm{AF}^{1}$.

\section{Limitations}

This study is the first study to assess the effect of AF on regulation of vascular tone in LA and LV arteries. We have studied only one, relatively early time point in the pathogenesis of AF, and a different behavior may develop in later stages. We have studied isolated vessels in a perfusion chamber, and have observed clear differences between the groups in vascular responsiveness to a selected panel of vasoactive agents. However, in vivo, the vascular tone will be determined not only by this responsiveness but also by the balance between vasoconstrictors and vasodilators present in the tissue. Nevertheless, altered behavior of isolated vessels provides important clues to the regulation of vascular tone in vivo.

Our study is descriptive and phenomenological; further studies will have to elucidate the mechanism responsible for altered vascular responsiveness. For example, the increased force generated during receptor-independent vasoconstriction could result from either increased calcium influx through membrane channel, increased mobilization from intracellular stores and/ or enhanced sensitivity of the contractile apparatus to calcium. We observed an increased sensitivity to bradykinin, without a change in sensitivity to the direct NO donor SNP. In principle, these results could explained by increased $\mathrm{NO}$ generation by endothelial cells, or by increased production or/ sensitivity to the other mediators of endothelium-dependent vasodilation, prostaglandins and EDHF. 


\section{REFERENCES}

1. van Bragt KA, Nasrallah HM, Kuiper M, Luiken JJ, Schotten U, Verheule S. Atrial supplydemand balance in healthy adult pigs: coronary blood flow, oxygen extraction, and lactate production during acute atrial fibrillation. Cardiovascular research 2014;101:9-19.

2. van Bragt KA, Nasrallah HM, Kuiper M, van Hunnik A, Kuijpers NH, Schotten U, Verheule S. Dynamic regulation of atrial coronary blood flow in healthy adult pigs. Heart rhythm : the official journal of the Heart Rhythm Society 2015;12:991-1000.

3. Gramley F, Lorenzen J, Jedamzik B, Gatter K, Koellensperger E, Munzel T, Pezzella F. Atrial fibrillation is associated with cardiac hypoxia. Cardiovascular pathology : the official journal of the Society for Cardiovascular Pathology 2010;19:102-111.

4. Ausma J, Coumans WA, Duimel H, Van der Vusse GJ, Allessie MA, Borgers M. Atrial high energy phosphate content and mitochondrial enzyme activity during chronic atrial fibrillation. Cardiovascular research 2000;47:788-796.

5. Thijssen VL, van der Velden HM, van Ankeren EP, Ausma J, Allessie MA, Borgers M, van Eys $\mathrm{GJ}$, Jongsma HJ. Analysis of altered gene expression during sustained atrial fibrillation in the goat. Cardiovascular research 2002;54:427-437.

6. Mulvany MJ, Halpern W. Contractile properties of small arterial resistance vessels in spontaneously hypertensive and normotensive rats. Circulation research 1977;41:19-26.

7. Halpern W, Mulvany MJ, Warshaw DM. Mechanical properties of smooth muscle cells in the walls of arterial resistance vessels. The Journal of physiology 1978;275:85-101.

8. Chilukoti RK, Mostertz J, Bukowska A, Aderkast C, Felix SB, Busch M, Volker U, Goette A, Wolke C, Homuth $\mathrm{G}$, Lendeckel U. Effects of irbesartan on gene expression revealed by transcriptome analysis of left atrial tissue in a porcine model of acute rapid pacing in vivo. International journal of cardiology 2013;168:2100-2108.

9. Folkow B. Physiological aspects of primary hypertension. Physiological reviews 1982;62:347504 .

10. Hirano K, Hirano M, Kanaide H. Regulation of myosin phosphorylation and myofilament $\mathrm{Ca}+$ sensitivity in vascular smooth muscle. Journal of smooth muscle research $=$ Nihon Heikatsukin Gakkai kikanshi 2004;40:219-236.

11. Bowles DK, Hu Q, Laughlin MH, Sturek M. Exercise training increases L-type calcium current density in coronary smooth muscle. The American journal of physiology 1998;275:H21592169 .

12. Nguyen BL, Fishbein MC, Chen LS, Chen PS, Masroor S. Histopathological substrate for chronic atrial fibrillation in humans. Heart rhythm : the official journal of the Heart Rhythm Society 2009;6:454-460. 
13. Chang CM, Wu TJ, Zhou S, Doshi RN, Lee MH, Ohara T, Fishbein MC, Karagueuzian HS, Chen PS, Chen LS. Nerve sprouting and sympathetic hyperinnervation in a canine model of atrial fibrillation produced by prolonged right atrial pacing. Circulation 2001;103:22-25.

14. Jayachandran JV, Sih HJ, Winkle W, Zipes DP, Hutchins GD, Olgin JE. Atrial fibrillation produced by prolonged rapid atrial pacing is associated with heterogeneous changes in atrial sympathetic innervation. Circulation 2000;101:1185-1191.

15. Duncker DJ, Bache RJ. Regulation of coronary vasomotor tone under normal conditions and during acute myocardial hypoperfusion. Pharmacology \& therapeutics 2000;86:87-110.

16. Folta A, Joshua IG, Webb RC. Dilator actions of endothelin in coronary resistance vessels and the abdominal aorta of the guinea pig. Life sciences 1989;45:2627-2635.

17. Rubanyi GM, Polokoff MA. Endothelins: molecular biology, biochemistry, pharmacology, physiology, and pathophysiology. Pharmacological reviews 1994;46:325-415.

18. Mayyas F, Niebauer M, Zurick A, Barnard J, Gillinov AM, Chung MK, Van Wagoner DR. Association of left atrial endothelin-1 with atrial rhythm, size, and fibrosis in patients with structural heart disease. Circulation Arrhythmia and electrophysiology 2010;3:369-379.

19. Tuinenburg AE, Van Veldhuisen DJ, Boomsma F, Van Den Berg MP, De Kam PJ, Crijns HJ. Comparison of plasma neurohormones in congestive heart failure patients with atrial fibrillation versus patients with sinus rhythm. The American journal of cardiology 1998;81:1207-1210.

20. Ungvari Z, Koller A. Endothelin and prostaglandin H(2)/thromboxane $A(2)$ enhance myogenic constriction in hypertension by increasing $\mathrm{Ca}(2+)$ sensitivity of arteriolar smooth muscle. Hypertension 2000;36:856-861.

21. Hattori Y, Kawasaki H, Kanno M. Increased contractile responses to endothelin-1 and U46619 via a protein kinase C-mediated nifedipine-sensitive pathway in diabetic rat aorta. Research communications in molecular pathology and pharmacology 1999;104:73-80.

22. Vanhoutte PM. Endothelium-dependent hyperpolarizations: the history. Pharmacological research 2004;49:503-508.

23. Tinker JH, Michenfelder JD. Sodium nitroprusside: pharmacology, toxicology and therapeutics. Anesthesiology 1976;45:340-354.

24. Muller JM, Myers PR, Laughlin MH. Vasodilator responses of coronary resistance arteries of exercise-trained pigs. Circulation 1994;89:2308-2314.

25. Thompson MA, Henderson KK, Woodman CR, Turk JR, Rush JW, Price E, Laughlin $\mathrm{MH}$. Exercise preserves endothelium-dependent relaxation in coronary arteries of hypercholesterolemic male pigs. Journal of applied physiology 2004;96:1114-1126.

26. Gschwend S, Buikema H, Henning RH, Pinto YM, de Zeeuw D, van Gilst WH. Endothelial 
dysfunction and infarct-size relate to impaired EDHF response in rat experimental chronic heart failure. European journal of heart failure 2003;5:147-154.

27. Hambrecht R, Adams V, Erbs S, Linke A, Krankel N, Shu Y, Baither Y, Gielen S, Thiele H, Gummert JF, Mohr FW, Schuler G. Regular physical activity improves endothelial function in patients with coronary artery disease by increasing phosphorylation of endothelial nitric oxide synthase. Circulation 2003;107:3152-3158.

28. Reilly SN, Jayaram R, Nahar K, Antoniades C, Verheule S, Channon KM, Alp NJ, Schotten U, Casadei $\mathrm{B}$. Atrial sources of reactive oxygen species vary with the duration and substrate of atrial fibrillation: implications for the antiarrhythmic effect of statins. Circulation 2011;124:1107-1117. 



\section{Early metabolic and}

\section{mitochondrial remodeling in a}

pig model of atrial fibrillation

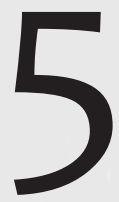

Philippe Pasdois*; Hussein M Nasrallah*; Marion Kuiper; Bertrand Beauvoit; Joost Luiken; Virginie Loyer; Pierre Dos Santos; Uwe Lendeckel; Lorenz Schild; Pierre Jaïs; Ulrich Schotten; Sander Verheule.

* The first two authors contributed equally to this chapter 


\section{ABSTRACT}

Background: The high activation rates during atrial fibrillation (AF) increase atrial oxygen demand. We have previously shown that this causes supply/demand ischemia during acute AF. Here we investigated AF-induced remodelling of mitochondrial function after one week of induced AF.

Methods: Using a right atrial pacemaker lead, AF was maintained by rapid atrial pacing (RAP) for one week in 7 Landrace pigs; 7 normal healthy pigs served as controls. Following the RAP period, pigs were anesthetized and intubated and the chest was opened to obtain snap frozen tissue and collect tissue (atria and ventricle) to perform structural, biochemical and functional studies. RAP-induced mitochondrial structural remodelling was studied by electron microscopy while mitochondrial function was studied in suspensions of isolated mitochondria.

Results: After one week of RAP, the phosphocreatine to Pi ratio was decreased when compared to the control group. This was associated with a mitochondrial size reduction and with a significant decrease of the total cardiolipin $(\mathrm{CL})$ content. Although the ratio of oxidized $C L$ to total $C L$ was increased, mitochondrial hydrogen peroxide production was decreased. RAP mitochondria had a lower proton leak and a higher ADP stimulated respiration, independent of the respiratory substrates used. During fatty acid oxidation and under phosphorylating conditions, RAP mitochondria had a depolarized membrane potential. The mitochondrial permeability transition pore was more sensitive to calcium in RAP mitochondria, but only when fatty acids were used as respiratory substrates. RAP atria were characterized by a marked glucose-6-phosphate accumulation and an increase of bound mitochondrial hexokinase activity suggesting an impairment of glucose metabolism.

Conclusions: One week of AF causes mitochondrial remodelling at the functional and structural level. These phenomena may contribute to the atrial structural remodelling observed during AF progression. 


\section{INTRODUCTION}

Atrial fibrillation (AF) is a common arrhythmia characterized by a fast and irregular activation of the atria. Animal studies of AF maintained by rapid atrial pacing (RAP) have demonstrated that $A F$ in itself promotes $A F^{1}$. Thus, patients with paroxysmal $A F$ have a significant risk to develop persistent or even permanent form of the disease ${ }^{2}$. Despite extensive research, the mechanisms responsible for the progression of AF are still not fully understood. Typical features of AF are electrical ${ }^{1}$, contractile ${ }^{3}$ and structural remodelling ${ }^{4}$. An important aspect of electrical remodelling, the down-regulation of the calcium current, seems to be dependent on the alteration of calcium homeostasis ${ }^{5}$. The contractile dysfunction could be secondary to myocyte dedifferentiation towards a phenotype similar to the hibernating ventricular myocardium ${ }^{6}$. It has recently been proposed that the structural remodelling could be partly controlled by the redox state of the atria ${ }^{7}$. AF is also characterized by an impairment of the atrial energetic status ${ }^{8}$ and by a metabolic remodelling leading to glycogen accumulation ${ }^{9}$. Some of the AF characteristics described above might be caused by a mitochondrial dysfunction. For instance, it has been shown that 1 week of AF leads to calcium accumulation in the cytosolic as well as in the mitochondrial compartment ${ }^{10}$. Proteomics and metabolomics studies have shown that patients with persistent AF were characterized by a metabolic switch towards the utilization of ketone bodies as substrates for mitochondrial tricarboxylic acid cycle ${ }^{11}$. Reactive oxygen species (ROS) overproduction has been proposed to play a role in the development of AF secondary to their direct effect on ion channels (electrical remodelling) and signalling pathways involved in structural remodelling 7 . It is interesting to note that some of the typical features of AF (i.e. cellular energetics impairment, ROS and $\mathrm{Ca}^{2+}$ overload) are known factors activating directly or indirectly the mitochondrial permeability transition pore (mPTP) ${ }^{12}$. This non-specific channel localized in the inner mitochondrial membranes is known for its critical role in regulating life and death of the cell ${ }^{13}$. Enhanced channel opening might contribute to the pathological remodelling of the heart ${ }^{14}$. A recent report suggests that this channel might be involved in the development of post-operative AF in patients with metabolic syndrome undergoing coronary artery bypass grafting ${ }^{15}$. Thus a growing body of evidence points towards the fact that mitochondrial dysfunction could be considered as an arrhythmogenic condition.

In animal models and human atrial biopsies, AF is characterized by an impairment of cellular energetics ${ }^{8}$ The etiology of this phenomenon is not entirely clear and could result from an diminished ATP synthesis and / or an increased energy demand. Using a goat model of rapid atrial pacing, Ausma and colleagues have argued that the energetic depletion was not secondary to an ischemic insult but rather correspond to an increased energetic demand ${ }^{8}$. In contrast, we have shown that acute AF in pigs leads to a supply-demand mismatch corresponding to a mild ischemic insult ${ }^{16}$. 
In absence of ischemia, the mitochondrial compartment should match the increased energetic demand caused by AF. The literature demonstrates that this is not the case, implying that mitochondria are unable to fulfil one their major functions. It is possible that AF causes alterations in mitochondrial function and/or structure, impairing their capacity to match an increased energetic demand. Thus, in this study we have used a pig model of rapid atrial pacing (RAP) to investigate the impact of 1 week of AF on metabolic and mitochondrial remodelling.

\section{METHODS}

\section{Animal model}

Dutch Landrace pigs weighing $60 \pm 5 \mathrm{~kg}$ were used ( $\mathrm{n}=7$ for the control group and $\mathrm{n}=7$ for the RAP group). All animal procedures were in accordance with national and institutional guidelines and approved by the local ethical committee. Animals in the RAP group were premedicated with Zoletil $(5-8 \mathrm{mg} / \mathrm{kg} \mathrm{im}$ ) and anaesthesia was induced with Thiopenthal (5-15 mg/kg iv). After adequate sedation animals were intubated and mechanically ventilated $\left(\mathrm{O}_{2}\right.$ saturation $90-100 \%$; pressure $15-20 \mathrm{CmH}_{2} \mathrm{O}$ ). Anaesthesia maintained with Midazolam ( $0.5 \mathrm{mg} / \mathrm{Kg} / \mathrm{h}$ iv) and Fentanyl ( $3 \mu \mathrm{g} / \mathrm{Kg} / \mathrm{h}$ iv). Before starting the surgical procedure a single dose of Ampicilline (1g iv) was administered as perioperative antibiotic prophylaxis. The jugular vein was prepared and a pacemaker lead (J-Leads Capsurefix 5568 - $53 \mathrm{~cm}$, Medtronic Inc. Minneapolis, Minnesota) was placed in the right atrial appendage under fluoroscopic guidance. The pacing threshold was determined using external pacemaker. The jugular vein was closed and the lead fixed with $\mathrm{R}$ knots. The pacemaker (Itrel, Enpulse Insync or Kappa, Medtronic) was placed in a pocket below the musculus sternocleidomastoideus and connected to the lead. The different wound layers were closed with resorbable Polysorb 2-o (subcutaneous) and resorbable Polysorb 3-o (cutaneous). Up to 2 days after surgery, Buprenorphine (0.02 mg/kg I.M., twice daily) was given up to two days after surgery and ampicillin (20mg/kg I.M., once daily) was given for three days after surgery. After a recovery period of one week, the implanted pacemaker was switched on at a rate of 600 beats per minute at $4 \mathrm{x}$ threshold for pacing was maintained for one week.

\section{Sacrifice experiments and tissue collection}

In the RAP group the pacemaker was switched off. Anaesthesia was induced and maintained as described above. After intubation and the start of mechanical ventilation, a left lateral incision was made, the fifth rib removed and the pericardium opened to expose the left side of the heart. In the RAP group, the pacemaker was switched on again. Tissue collection was started after an equilibration period of 30 minutes.

A custom built clamp cooled in liquid nitrogen was used to freeze atrial and 
ventricular tissue directly in the chest of the animals in order to biochemically assesses the energetic status of the respective heart chambers. Hearts were then excised and pieces of the left atrial appendage (LA) and of the left ventricle (LV) were immediately collected and immersed in a cold mitochondrial isolation buffer. Tissues samples from the four different cardiac chambers were immersed in glutaraldehyde fixative solution for further processing and ultra structural analysis by electron microscopy.

\section{Isolation of mitochondria}

Pieces of the LA and LV were rapidly chopped with scissors before incubating them at $4^{\circ} \mathrm{C}$ for $7 \mathrm{~min}$ with stirring in $25 \mathrm{ml}$ of mitochondrial isolation buffer (ISA: $300 \mathrm{mM}$ sucrose, $2 \mathrm{mM}$ EGTA, $10 \mathrm{mM}$ Tris- $\mathrm{HCl}$, pH 7.1 at $4^{\circ} \mathrm{C}$ ) containing $0.1 \mathrm{mg} / \mathrm{ml}$ of bacterial proteinase type XXIV (Sigma). The resulting tissue suspension was poured into a 50 $\mathrm{ml}$ glass Potter homogenizer and homogenized for 3 min using a motorized Teflon pestle. The homogenate was centrifuged at 7,500 $\mathrm{g}$ for $7 \mathrm{~min}$ and the resulting pellet rinsed twice with $5 \mathrm{ml}$ ISA (supplemented with $2 \mathrm{mg} / \mathrm{ml}$ of Free Fatty Acid Bovine Serum Albumin, ISA-BSA), resuspended in $20 \mathrm{ml}$ ISA-BSA and subject to further homogenization as described above. The homogenate was then centrifuged at $700 \mathrm{~g}$ for $10 \mathrm{~min}$ and the resultant supernatant centrifuged at 7,000 $\mathrm{g}$ for $10 \mathrm{~min}$ to yield a crude mitochondrial pellet that was resuspended in ISA-BSA containing $25 \%$ $(\mathrm{w} / \mathrm{v})$ Percoll $\left(\mathrm{pH} 7.1-7.2\right.$ at $\left.4^{\circ} \mathrm{C}\right)$ and centrifuged at $17,000 \mathrm{~g}$ for $10 \mathrm{~min}$. The resulting pellet was resuspended with ISA-BSA and centrifuged again at 7,000 $\mathrm{g}$ for $10 \mathrm{~min}$. The final purified mitochondrial pellet was resuspended in ISA-BSA and the protein concentration determined by a bicinchoninic acid based assay (Pierce ${ }^{T M}$ BCA protein assay kit) according to the manufacturer specification and using BSA as a standard. Mitochondria were kept on ice at a final concentration of $80 \mathrm{mg} / \mathrm{ml}$ for no more than 5 hours. Aliquots of mitochondria were frozen in liquid nitrogen for further biochemical analysis.

\section{Measurements of respiration}

Oxygen consumption of isolated mitochondria was measured at $30^{\circ} \mathrm{C}$ in a buffer containing $\mathrm{KCl} 125 \mathrm{mM}$, MOPS 20mM, Tris $10 \mathrm{mM}$, EGTA $10 \mu \mathrm{M}, \mathrm{KH}_{2} \mathrm{PO}_{4} 2.5 \mathrm{mM}, \mathrm{MgCl}_{2}$ $2.5 \mathrm{mM}, 2 \mathrm{mg} / \mathrm{ml} \mathrm{BSA}, \mathrm{pH} 7.3$ at room temperature with $\mathrm{KOH}$. Rates of respiration were determined before and after addition of $1.5 \mathrm{mM}$ ADP (State 2 and State 3 respectively) in the presence of glutamate $(5 \mathrm{mM})$ and malate $(2 \mathrm{mM})$ or PalmitoylCarnitine $(10 \mu \mathrm{M})$ and malate $(1 \mathrm{mM})$. The permeability of the mitochondrial outermembrane for cytochrome $c$ was assessed by addition of exogenous cytochrome $c(10 \mu \mathrm{M})$ after ADP. Rates of oxygen consumption were expressed as nanomol of oxygen used per min and per unit of citrate synthase (nmol $\mathrm{O}_{2} / \mathrm{min} / \mathrm{U}$ of CS). 


\section{Hydrogen peroxide emission in isolated mitochondria}

The rate of $\mathrm{H}_{2} \mathrm{O}_{2}$ emission in isolated mitochondria was determined with the fluorescent hydrogen peroxide indicator, Amplex Red $(10 \mu \mathrm{mol} / \mathrm{L})$ in presence of peroxidase $\left(10 \mu \mathrm{g} / \mathrm{ml}\right.$ ) in a $\mathrm{KCl}$-based respiration buffer (see above) at $30^{\circ} \mathrm{C}$ as described previously ${ }^{17}{ }^{18}$. Briefly, in presence of hydrogen peroxide the peroxidase catalyses the oxidation of amplex red to form the fluorescent compound resofurin. Resofurin was excited at $550 \mathrm{~nm}$ and fluorescence was collected at 590 $\mathrm{nm}$. A calibration curve was recorded to quantify mitochondrial $\mathrm{H}_{2} \mathrm{O}_{2}$ emission. The rate of reactive oxygen species production was assessed in absence or presence of $1.5 \mathrm{mM}$ ADP. The maximal rate of mitochondrial $\mathrm{H}_{2} \mathrm{O}_{2}$ emission was assessed by addition of the complex III inhibitor Antimycin-A ( $5 \mu \mathrm{mol} / \mathrm{L}$ ). Rates of $\mathrm{H}_{2} \mathrm{O}_{2}$ emission were expressed as picomol of hydrogen peroxide per minute and per unit of citrate synthase ( $\mathrm{pmol} \mathrm{H}_{2} \mathrm{O}_{2} / \mathrm{min} / \mathrm{U}$ of $\mathrm{CS}$ ).

\section{Mitochondrial membrane potential $\left(\Delta \Psi_{\mathrm{m}}\right)$}

Mitochondria were incubated as described above in presence of 50nM of rhodamine 123 in a $\mathrm{KCl}$ based respiration buffer (see above). $\Delta \Psi_{\mathrm{m}}$ was monitored in presence of respiratory substrates alone and $1.5 \mathrm{mM}$ ADP was then added. The fluorescence recorded was normalized to the fluorescence obtained after addition of $5 \mu \mathrm{M}$ of the adenine nucleotides translocator inhibitor carboxyatractyloside, CAT (i.e. $100 \%$ reduced, minimal value) followed by $2 \mu \mathrm{mol} / \mathrm{L}$ of the mitochondrial uncoupler trifluorocarbonylcyanide Phenylhydrazone, FCCP (i.e. $100 \%$ oxidized, maximal value). $\Delta \Psi_{m}$ in basal condition and following addition of a saturating quantity of ADP were expressed as a percentage normalised to the minimal and maximal values.

\section{Mitochondrial calcium retention capacity assay}

Mitochondria were incubated at $30^{\circ} \mathrm{C}$ in the following buffer $\mathrm{KCl} 125 \mathrm{mM}$, MOPS 20mM, Tris 10mM, EGTA $10 \mu \mathrm{M}, \mathrm{KH}_{2} \mathrm{PO}_{4} 2 \mathrm{mM}, \mathrm{pH} 7.3$ adjusted at room temperature with $\mathrm{KOH}$. Extra-mitochondrial calcium was monitored using the fluorescent dye calcium green at a final concentration of $1 \mu \mathrm{mol} / \mathrm{L}$. Calcium spikes were added successively until mitochondria could not buffer calcium in the medium, thus reflecting the maximum calcium retention capacity $(C R C)$ before MPTP opening. $A$ calibration curve with known calcium concentration was performed to quantify the total extra-mitochondrial calcium. CRC was expressed as micromolar of free calcium per unit of citrate synthase ( $\mu \mathrm{M}$ free $\mathrm{Ca}^{2+} / \mathrm{U}$ of $\mathrm{CS}$ ). The free calcium concentration in the buffer was calculated taking into account the dissociation constant of the different calcium ligand, the quantity of metals and the $\mathrm{pH}$ of the assay buffer used. To assess the average mitochondrial calcium uptake velocity as a function of calcium quantity in the buffer, the area under each peak was integrated and calibrated. The average mitochondrial calcium uptake velocity was then expressed as micromolar of 
total calcium per $\min \left(\mu \mathrm{M}\right.$ tot $\left.\mathrm{Ca}^{2+} / \mathrm{min}\right)$.

\section{Preparation of freeze-clamped deproteinized samples}

Pieces of frozen LA or LV (50 to 100mg) were placed in Eppendorf $(2.5 \mathrm{ml})$ containing one tungsten bead. Tubes were then installed in Teflon blocs, pre-cooled in liquid $\mathrm{N}_{2}$, of a mixer mil (Retsch MM400). Frozen pieces were pulverized by 4 cycles of $3 \mathrm{sec}$ interspaced by $15 \mathrm{sec}$ at a frequency of $30 \mathrm{~Hz}$. Ice-cold perchloric acid at $10 \%$ containing $25 \mathrm{mM}$ EDTA was added onto the heart powder to obtain an homogenate of $100-200 \mathrm{mg} / \mathrm{ml}$. The homogenate was then centrifuged for $2 \mathrm{~min}$ at 14,000g. The resulting supernatant was neutralized by successive addition of $\mathrm{KHCO}_{3} 2.4 \mathrm{~mol} / \mathrm{L}$ containing $10 \%$ of $\mathrm{KOH}-\mathrm{MOPS}$ until $\mathrm{pH}$ reaches 6.5 . The obtained solution was then centrifuged for 2 min at 14,00og and the supernatant was analysed for its content in ADP and ATP. For each heart compartment (i.e. left atria and ventricle) of each pigs used for this study, two independent acidic extractions have been performed in order to perform two independent measurements for each energetic intermediates. Aliquots of the extracted metabolites were stored at $-80^{\circ} \mathrm{C}$ for further biochemical analysis except for ATP and ADP which were measured just after extraction.

\section{Measurement of metabolites}

ADP, ATP and PCr in the neutralized samples were assessed by bioluminescence (Lumac luminometer M1500P). ATP and ADP assay were performed in parallel in order to calculate the ratio of these two energetic intermediates. For ATP assessment deproteinized samples $(5,10,15,20$ and $25 \mu \mathrm{L})$ were incubated for $10 \mathrm{~min}$ in a final volume of $50 \mu \mathrm{L}$ of a buffer containing (EDTA $2 \mathrm{mmol} / \mathrm{L}, \mathrm{K}^{+}$-acetate, $\mathrm{MgCl}_{2} 2 \mathrm{mmol} / \mathrm{L}$, $\mathrm{pH} 7.7$ with Tris). Just before readings $50 \mu \mathrm{L}$ of the reaction buffer (PerkinElmer Kit, Ref 6016736), diluted 2-fold with deionised water, was added. Subsequently, three successive readings were performed in the luminometer to assess signal stability. For ADP assessment the protocol was identical except that the 1omin incubation period was performed in presence of pyruvate kinase $(4 \mathrm{U} / \mathrm{ml})$ and phosphoenolpyruvate $(2.5 \mathrm{mM}$, Sigma) in order to convert ADP into ATP.

$\mathrm{PCr}$ and ATP assays were performed in the same specimens in order to calculate the ratio of these two energetic intermediates. For $\mathrm{PCr}$ assessment the protocol was identical except that the 10min incubation period was performed in presence of creatine kinase ( $4 \mathrm{U} / \mathrm{ml}$, Roche, from rabbit muscle) and ultrapure ADP ( $35 \mu \mathrm{M}$ final concentration, CellTechnology) in order to convert PCr into ATP. Standard curves with known concentration of ATP or PCr were used in order to calibrate the experiments. ATP, ADP and PCr contents were expressed as $\mu$ moles per g wet weight.

\section{Glucose-6-phosphate (G6-P) and inorganic phosphate (Pi) content}

To determine the glucose-6-phosphate (G6-P) content in deproteinized samples, 100 
and $200 \mu \mathrm{L}$ of neutralized sample were incubated for $1 \mathrm{~min}$ at room temperature in $1 \mathrm{ml}$ final of assay buffer $\left(\mathrm{KH}_{2} \mathrm{PO}_{4} 50 \mathrm{mM}, \mathrm{NADP}^{+} 0.4 \mathrm{mM}, \mathrm{pH} 7.4\right.$ at room temperature). The reaction was started by addition of $0.5 \mathrm{U} / \mathrm{ml}$ of glucose-6-phosphate dehydrogenase and stopped when the optic density measured at 340nm reached a plateau. G6-P content of the deproteinized samples was expressed as $\mu$ moles / g wet weight by using the NADPH molar extinction coefficient.

To determine the inorganic phosphate $(\mathrm{Pi})$ content in deproteinized samples, 2.5, 5 and $10 \mu \mathrm{L}$ of neutralized sample were incubated for 20min at room temperature in $0.5 \mathrm{ml}$ final of assay buffer (Molybdate $9.6 \mathrm{mmol} / \mathrm{L}$, Malachite green $192 \mu \mathrm{mol} / \mathrm{L}$, Monidel P40 0.09\%). The optic density was then read at $610 \mathrm{~nm}$. A standard curve was performed with known concentration of $\mathrm{KH}_{2} \mathrm{PO}_{4}$ and total inorganic phosphate content was expressed as $\mu$ moles per wet weight.

\section{Determination of Hexokinase and Citrate synthase}

Aliquots $(0.75 \mathrm{mg}$ protein) of frozen isolated mitochondria prepared by the protease method (see above) were solubilized by sonication (10sec) in a buffer containing $33 \mathrm{mmol} / \mathrm{L} \mathrm{KH}_{2} \mathrm{PO}_{4}, 50 \mu \mathrm{mol} / \mathrm{L}$ dithiothreitol and proteases inhibitors (F. Hoffman-La Roche AG, cOmplete), pH 7.2 at room temperature. The homogenate concentration was adjusted to $2 \mathrm{mg} / \mathrm{ml}$ for mitochondrial hexokinase (mtHK) assay and $0.2 \mathrm{mg}$ / $\mathrm{ml}$ for citrate synthase (CS) assay. For mtHK assay samples (75, 100 or $150 \mu \mathrm{l})$ were added to $1 \mathrm{ml}$ assay buffer ( $\mathrm{pH} 7.4$ at room temperature) containing $100 \mathrm{mmol} / \mathrm{L}$ $\mathrm{KH}_{2} \mathrm{PO}_{4}, 0.4 \mathrm{mmol} / \mathrm{L} \mathrm{NADP}^{+}, 10 \mathrm{mmol} / \mathrm{L} \mathrm{MgCl}_{2}, 5 \mathrm{mmol} / \mathrm{L} \mathrm{ATP}, 0.3 \%$ (weight / v) Triton $\mathrm{X}-100$ and $0.5 \mathrm{U} / \mathrm{ml}$ of glucose-6-phosphate dehydrogenase and incubated for $2 \mathrm{~min}$ at RT before addition of glucose (1mM final concentration) to start the reaction. Hexokinase activity was calculated from the rate of NADPH production corrected for glucose-independent rates of NADPH formation determined in parallel assays lacking glucose. For assay of citrate synthase mitochondrial samples (20, 30 or $40 \mu \mathrm{l}$ of $0.2 \mathrm{mg}$ protein $/ \mathrm{ml}$ ) were added to $1 \mathrm{ml}$ assay buffer ( $\mathrm{pH} 7.4$ at room temperature) containing 50mM KH $\mathrm{PO}_{4}, 0.3 \%$ (weight / v) Triton X-100 and $150 \mu \mathrm{M}$ 5,5'-dithiobis-2nitrobenzoic acid (DTNB) and incubated for $2 \mathrm{~min}$ in the presence of $0.3 \mathrm{mM}$ acetyl$\mathrm{CoA}$, before addition of oxaloacetic acid $(500 \mu \mathrm{mol} / \mathrm{L}$ final) to start the reaction. One unit of citrate synthase was defined as equal to the reduction of $1 \mu$ mole DTNB per min.

\section{Western-blotting}

Proteins were separated by SDS-PAGE on 4-12\% Bis-Tris Standard XT precast gels (BioRad) and moved to nitrocellulose membranes. The membranes were probed with the appropriate primary antibodies based on instructions provided by manufacturer. The protein bands were visualized using enhanced chemiluminescence, and the immunoblot intensities were quantified using Bio-Rad Quantity One. Antibodies 
used to assess the level of the type 4 glucose transporter (Glut-4), primary antibody Chemicon international Ab1346, diluted at 1 in 4000 and secondary antibody DarPo, Chemicon AP182P, diluted at 1 in 4000. Antibodies used to assess the faty acids transporter (CD36), primary antibody provided as a gift by N. Tandon numberMO25, diluted at 1 in 20000 and secondary antibody RamPo, Dakocytomation P0161 diluted at 1 in 20000. Antibodies used to assess the type 1 glucose transporter (Glut-1), primary antibody Abcam ab652 diluted at 1 in 1000, secondary antibody DarPo, Jackson Immunolaboratories Inc, 711-035-152 diluted at 1 in 9000. AMPK-alpha monoclonal antibody diluted at 1 in 1000 (\#2532, cell signalling technology, Inc. USA). PAMPK monoclonal antibody diluted at 1 in 1000 (\#2535 cell signalling technology Inc. USA).

\section{Quantification of Cardiolipin species by LS-MS/MS}

$C L$ was extracted from the samples by a modified Folch extraction procedure ${ }^{19}$. Briefly, 5ong of tetra-myristoyl-CL [(C14:0) -CL; Avanti Polar Lipids, INC., Alabaster, USA] was added as internal standard to $10 \mu$ incubation mixture. The extraction of $C L$ was carried out with chloroform/methanol (2/1) containing $0.05 \%$ BHT as antioxidant. Aqueous and lipid phases were separated by adding 0.01M HCL followed by intensive shaking and subsequent centrifugation. The lower lipid phase was collected, dried under nitrogen atmosphere and acidified as described by Schlame et al. Ice-cold methanol ( $2 \mathrm{ml})$, chloroform $(1 \mathrm{ml})$ and $0.1 \mathrm{M} \mathrm{HCl}(1 \mathrm{ml})$ were added to the sample. The solution was intensively mixed and incubated for $5 \mathrm{~min}$ on ice. Phase separation was achieved by addition of $1 \mathrm{ml}$ of $\mathrm{CHCl}_{3}$ and $1 \mathrm{ml}$ of $0.1 \mathrm{M} \mathrm{HCl}$. The chloroform phase was recovered as $\mathrm{CL}$-containing sample. Finally, the sample was dried under nitrogen, dissolved in $0.8 \mathrm{ml} \mathrm{CHCl}_{3} / \mathrm{CH}_{3} \mathrm{OH} / \mathrm{H}_{2} \mathrm{O}(50 / 45 / 5, \mathrm{v} / \mathrm{v} / \mathrm{v})$, mixed, and filtered over $0.2 \mu \mathrm{m}$ PTFE membranes. This sample was ready for immediate Liquid chromatography mass spectrometry (LC-MS/MS) analysis according to Valianpour et al ${ }^{20}$.

Liquid chromatography mass spectrometry: A TSQ Quantum Discovery Max (Thermo Fisher Scientific GmbH, Dreieich, Germany) was used in the negative ion electrospray ionization (ESI) mode. The HPLC system consisted of a Surveyor MS quaternary narrow bore pump with integrated vacuum degasser and a Surveyor auto sampler. The auto sampler tray temperature was maintained at $8^{\circ} \mathrm{C} .10 \mu \mathrm{l}$ of the lipid extract dissolved in chloroform/methanol/water (50/45/5) was injected (in partial loop mode) and CL was separated by using a LiChroCart column (125 x 2 $\mathrm{mm}$ ), LiChrospher Si6o (5 $\mu \mathrm{m}$ particle diameter; Merck, Darmstadt, Germany) and a linear gradient between solution A (chloroform) and solution B (methanol/water, 9:1 $(\mathrm{v} / \mathrm{v}))$. Both solutions additionally contained $0.1 \mathrm{ml} / \mathrm{l}$ of $25 \%$ aqueous ammonia. The gradient was as follows: $0-0.2 \mathrm{~min}, 92 \% \mathrm{~A}, 8 \% \mathrm{~B}, 0.2-4.5 \mathrm{~min}, 92 \% \mathrm{~A}$ to $30 \% \mathrm{~A}$ and $8 \% \mathrm{~B}$ to $70 \% \mathrm{~B}, 4.5-6 \mathrm{~min}, 30 \% \mathrm{~A}, 70 \% \mathrm{~B}, 6-6.5 \mathrm{~min} 30 \% \mathrm{~A}$ to $92 \% \mathrm{~A}, 70 \% \mathrm{~B}$ to $8 \% \mathrm{~B}, 6.5-11 \mathrm{~min}, 92 \%$ $\mathrm{A}, 8 \% \mathrm{~B}$. The flow rate was $300 \mu \mathrm{l} / \mathrm{min}$. 
The total time of analysis was $11 \mathrm{~min}$. The eluate between 0.3 and $6 \mathrm{~min}$ was introduced into the mass spectrometer. Nitrogen was used as the nebulizing gas and argon was used as the collision gas at a pressure of $1.5 \mathrm{mTorr}$. The spray voltage was $3.5 \mathrm{kV}$, the ion source capillary temperature was set at $375^{\circ} \mathrm{C}$ and the cone-voltage was $30 \mathrm{~V}$. Daughter fragments from the doubly charged parent derived from (C18:2) $-\mathrm{CL}$ with $\mathrm{m} / \mathrm{z}$ (mass to charge ratio) $723.6((\mathrm{M}-2 \mathrm{H}) 2-12)$ were obtained using a collision energy of $36 \mathrm{eV}$. This molecular CL species and the internal standard ( $\mathrm{m} / \mathrm{z} 619.6)$ were analyzed by mass transfer reaction monitoring their doubly charged ions and their respective fatty acids linoleic acid m/z 279.2, and myristic acid m/z 227.2 using the selected reaction monitoring (SRM) mode. The same approach was used for parent and daughter fragments of other molecular CL species). The quantity of these molecular $\mathrm{CL}$ species was related to $(\mathrm{C} 18: 2)_{4}-\mathrm{CL}$.

To identify $C L$ species, we separated in prior experiments first $C L$ by thin layer chromatography. In the next step, we used the separated $C L$ to run parent scans for C16:0, C18:0, C18:1, C18:2, C18:3, C20:2, C20:3, C20:4 and C22:6 quantitatively occurring in goat heart. The parent peaks found were used to run product scans to find other fatty acid residues and to characterize CL composition. At the end of this procedure we identified nineteen $C L$ species listed in the tables. The analysis was performed in triplicate for each sample. Oxidized CL [(C18:2)3monohydroxylinoleic acid-CL] was measured in the SRM mode as a transition from m/z 731.6 to m/z 279.2 (linoleic acid). Internal cardiolipin standard ((C14:0 $\left.)_{4}-\mathrm{CL}\right)$ was from Avanti Polar Lipids (Alabaster, Alabama, USA) and the cardiolipin standard from bovine heart and all other reagents from Sigma (Munich, Germany).

\section{Electron microscopy}

For electron microscopy (EM) studies LA and LV tissue sections were gently sectioned into tiny chunks and stored in glutaraldehyde. As described previously fixed blocks were washed with $\mathrm{KH}_{2} \mathrm{PO}_{4}$ buffer containing $7.5 \%$ sucrose then post fixed with $2 \%$ $\mathrm{OsO}_{4}$ in $50 \mathrm{mM}$ veronal acetate buffer for $1 \mathrm{~h}$, dehydrated through graded ethanol series and routinely embedded in epoxy resin (Epon) ${ }^{4}$. Ultrathin sections were cut from each Epon sample and then counterstained with uranium acetate and lead citrate ${ }^{10}$. Sections were scanned at a magnification of 1900X using a Philips CM100 microscope. To determine mitochondrial size and number, 5 to 6 locations were photographed per animal from transversely sectioned myocytes. Nucleated areas were excluded. All analysis was blinded and performed using image J 1.46r, (National Institute of Health, USA).

\section{Statistical analysis}

All data are expressed as means \pm SEM and represent at least 5 independent preparations. All statistical analysis were performed with SPSS statistics 17.0. 
Unpaired samples were compared by a non-parametric Mann-Whitney test. Paired samples were compared by a paired student t-test. Statistical analysis of electron microscopy data was performed using a linear mixed model with Bonferroni correction. Results were considered statistically significant if the p-value was less than 0.05

\section{RESULTS}

\section{Energetic status}

The atrial energetic status was assessed in tissue samples directly frozen in the chest of the animals. This experimental approach was critical to avoid development of ischemia in the samples and the consequent hydrolysis of energetic intermediates. Following one week of AF, a significant decrease of the ATP (figure $1 A$ ) and $P C r$ (figure $1 B)$ contents was observed in the RAP LA when compared to the control LA. These changes were not associated with an increase in either ADP or inorganic phosphate contents (supplemental figures $1 \mathrm{~A}$ and $\mathrm{B}$ ). As a consequence, the ATP to ADP (Figure 1C) as well as the $\mathrm{PCr}$ to $\mathrm{Pi}$ (figure $1 \mathrm{D}$ ) ratios were significantly decreased in the RAP LA when compared to the control LA. In contrast, none of these parameters were affected in the left ventricle following one week of RAP (figure 1). The impaired energetic status was not associated to a modulation of the phosphorylation level of the major metabolic switch AMPK (figure $1 \mathrm{E}$ ) suggesting that RAP does not lead to severe ischemia.

\section{Substrate metabolism}

Because the mitochondrial compartment is the end-point of the glycolytic pathway and $\mathrm{AF}$ is characterized by glycogen accumulation, we assessed the content of glucose-6-phosphate (G-6-P, figure 2A). One week of AF promoted accumulation of G-6-P in the RAP LA when compared to control. The accumulation of this glycolytic intermediate was paralleled by an increase of the total mitochondrial hexokinase activity, further confirming that glucose metabolism is modulated by AF (figure $2 \mathrm{~B}$ ). This atrial glycolytic remodelling was not associated with a change in the expression level of the glucose transporters 1 or 4 in the RAP atria (figure $2 \mathrm{C}$ ). A recent study in a rabbit model showed that RAP leads to a downregulation of fatty-acid metabolism ${ }^{21}$, but in our model, one week of RAP does not lead to downregulation of CD36 (Figure $2 \mathrm{C}$ ). Following one week of RAP all the parameters described above were not altered in the ventricle (supplemental figure $2 \mathrm{~B}$ ) despite the absence of rhythm control.

\section{Mitochondrial oxygen consumption}

Following one week of maintained AF, mitochondria from LA and LV were isolated and purified on a Percoll gradient and their function was assessed. RAP significantly 

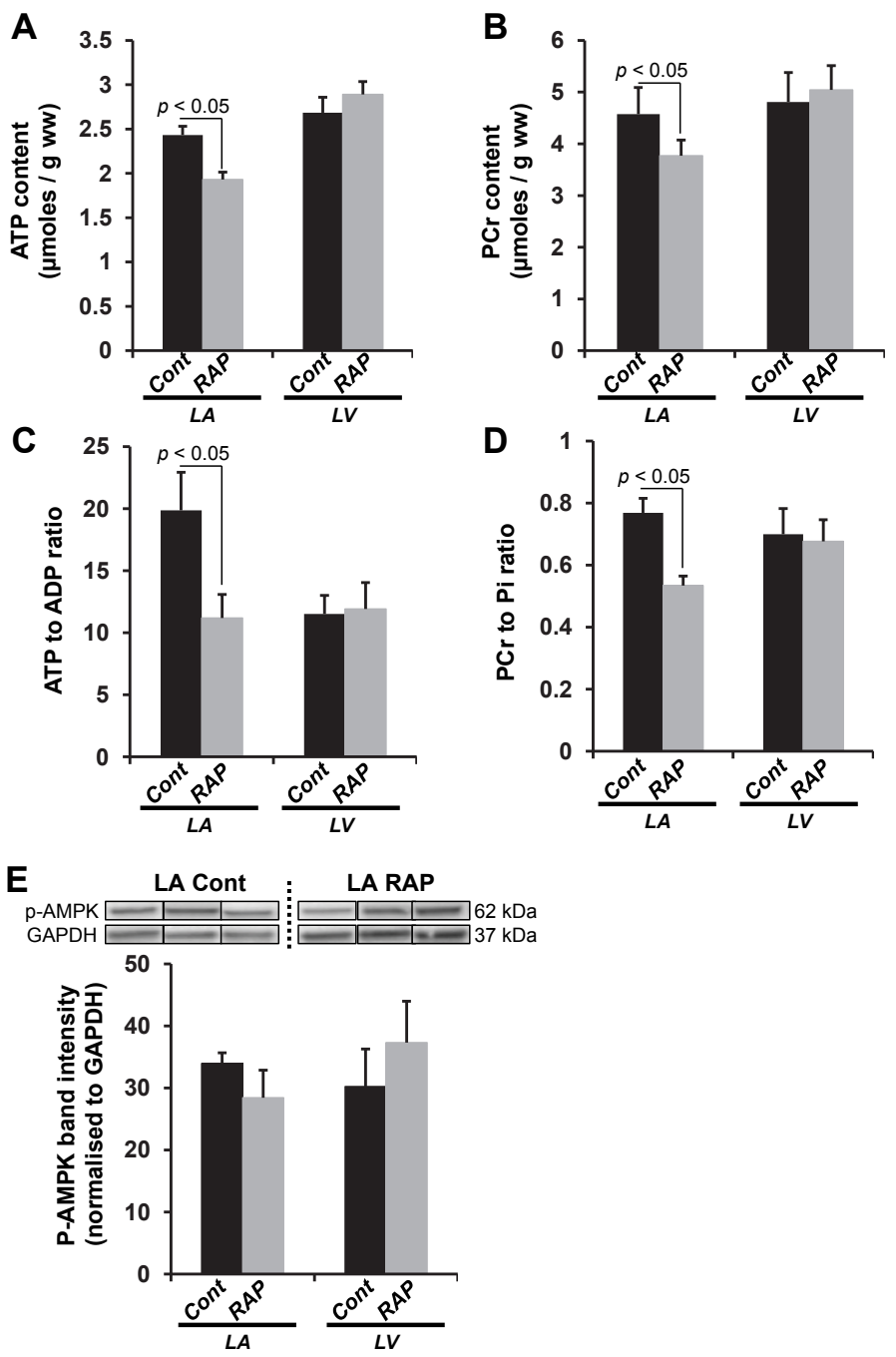

Figure 1. Effect of RAP on atrial and ventricular energetics

Pieces of LA and LV were snap-frozen in-vivo directly in the chest of the animal. Samples were then pulverized and an acidic extraction was performed to extract the energetic intermediates. Panels $\boldsymbol{A}$ and B: ATP (panel A) and PCr (panel B) contents were assessed as described in the methods section. Panels C and D: ATP to ADP (panel C) and PCr to Pi (panel D) ratios were calculated to assess the energetic alteration caused by AF. Panel E: Representative western-blots of the phosphorylated AMPK (P-AMPK) level and mean data normalized to the GAPDH content in the Cont and RAP groups. Data are presented in the bar graphs as means \pm SEM. $n=7$ independent preparation in each group.

decreased the LA mitochondrial basal respiration with palmitoyl-carnitine (PCM) as respiratory substrate (figure $3 A$ ). In contrast, LA mitochondria showed an increased ADP stimulated respiration in presence of glutamate + malate (GM) while no difference was observed in presence of PCM (figure 3B). These RAP-induced 
A

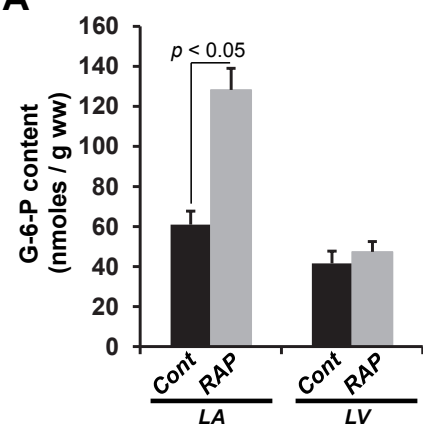

B

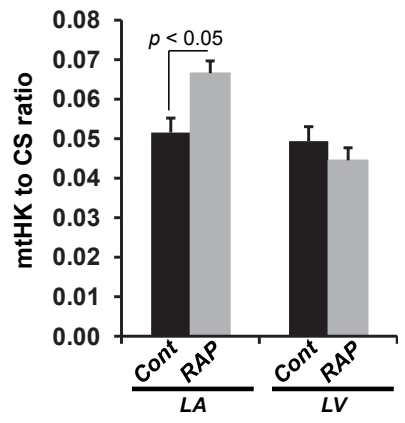

C
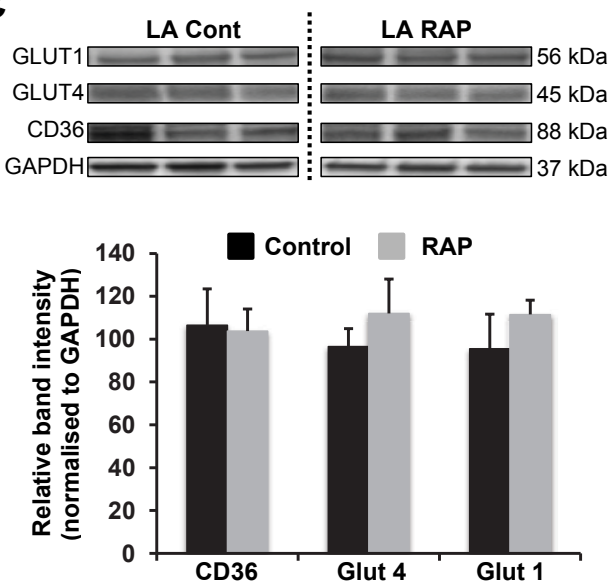

Figure 2. Effect of RAP on substrate metabolism

Panel A: Total atrial and ventricular glucose-6-phosphate (G-6-P) content assessed in the control and RAP groups. Panel B: Total atrial and ventricular mitochondrial hexokinase activity ( $\mathrm{mtHK}$ ) normalized to the citrate synthase (CS) content assessed in the control and ventricular groups. Panel C: Representative Western-blots of Glut 1\&4 and CD36 expression level in the atria and corresponding mean data normalized to the GAPDH content. Data are presented in the bar graphs as means \pm SEM. $n=7$ independent preparation in each group.

modulations of the basal and ADP stimulated respiration point towards an increase of the respiratory control in the LA mitochondria independently of the respiratory substrates used (figure $3 C$ ). Mitochondrial respiration in the LV was also affected by RAP. However, a parallel increase in the basal (figure 3A) and ADP stimulated (figure 3B) oxygen consumptions was observed in presence of $G M$ only. As a consequence, the respiratory control was unchanged in the RAP LV mitochondria when compared to the control LV mitochondria (figure $3 \mathrm{C}$ ). 

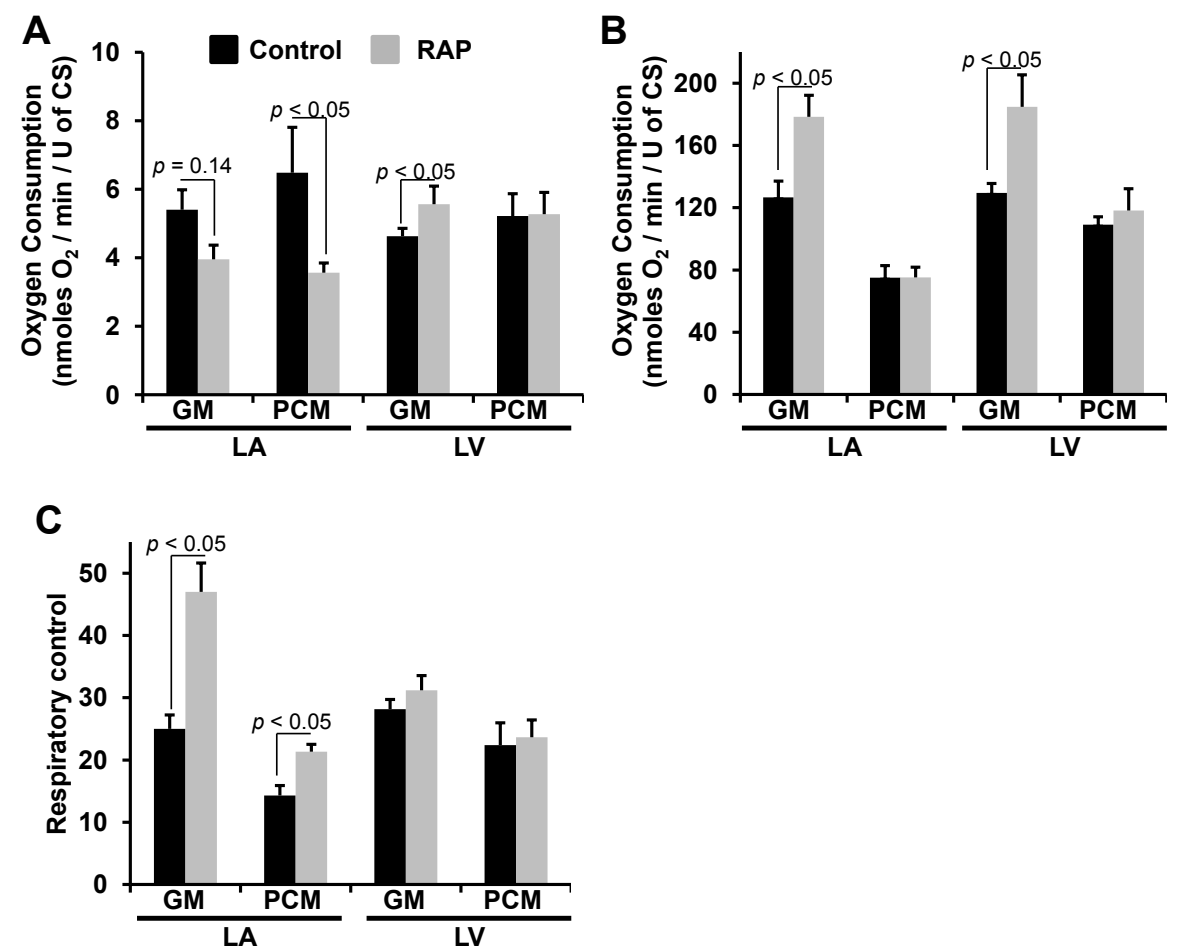

Figure 3. Effect of RAP on mitochondrial oxygen consumption

Mitochondria were isolated and their oxygen consumption was assessed in the control and RAP atria and ventricle. Oxygen consumption was measured in each group in presence of glutamate + malate $(G M)$ or in presence of palmitoyl-carnitine + malate (PCM). Panel A: Basal oxygen consumption. Panel B: Mitochondria were incubated in presence of a saturating concentration of ADP. Panel C: Mitochondrial respiratory control expressed as the ratio of the ADP stimulated respiration to the basal respiration. Data are presented as means $\pm S E M$. $n=5$ to 7 independent mitochondrial isolation.

\section{Mitochondrial membrane potential $(\Delta \Psi \mathrm{m})$}

Mitochondrial $\Delta \Psi_{\mathrm{m}}$ was qualitatively assessed using the fluorescent dye Rh123. Importantly, this experimental approach does not allow quantifying the absolute value of $\Delta \Psi_{\mathrm{m}}$ but enables to describe the behaviour of mitochondrial $\Delta \Psi_{\mathrm{m}}$ following addition of drugs. Typical traces of LA control and RAP mitochondria oxidizing PCM are shown in figure 4A. In absence of ADP, no differences were observed between RAP LA and control LA or RAP LV and control LV mitochondria (figure 4B). In contrast, following ADP addition RAP LA mitochondria oxidizing PCM were more depolarized than control LA mitochondria (figure 4C).

\section{Mitochondrial $\mathrm{H}_{2} \mathrm{O}_{2}$ emission}

One week of AF results, in presence of ADP, in a significant decrease of the mitochondrial hydrogen peroxide $\left(\mathrm{H}_{2} \mathrm{O}_{2}\right)$ emission in RAP LA mitochondria when compared to control LA mitochondria (Figure 5B). In the RAP LA mitochondria, 
A

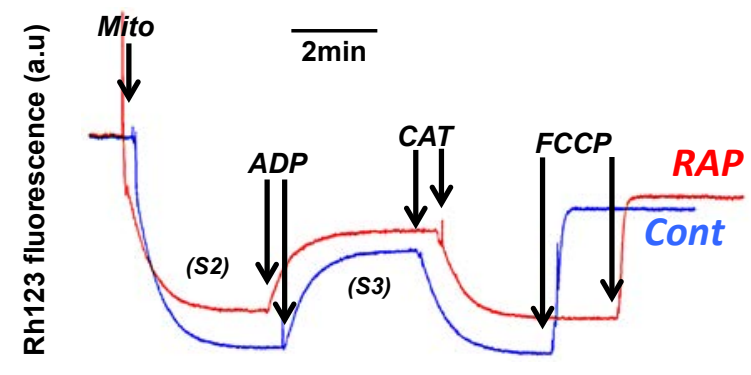

B

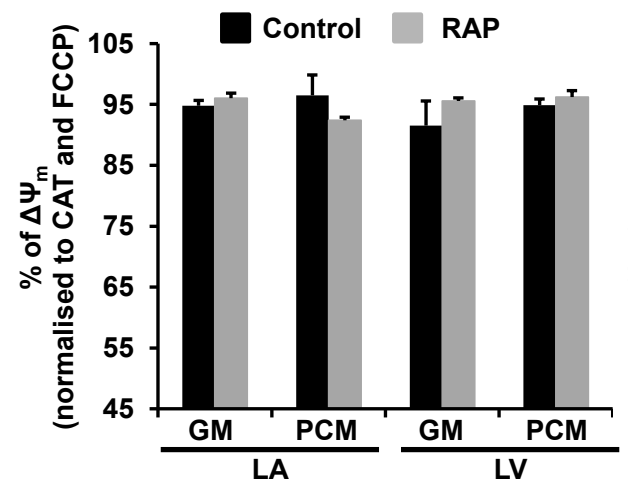

C

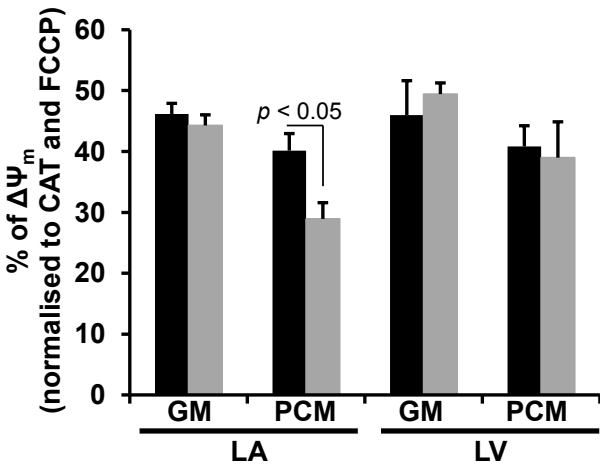

Figure 4. Effect of RAP on mitochondrial membrane potential $\left(\Delta \Psi_{m}\right)$ Mitochondria were isolated and their $\Delta \Psi_{m}$ was assessed in the control and RAP atria and ventricle. $\Delta \Psi_{m}$ was measured in each group in presence of glutamate + malate (GM) or in presence of palmitoylcarnitine + malate (PCM). Panel A: Typical recordings of mitochondrial $\triangle \Psi_{m}$ obtained in the Cont atria (Cont blue line) and RAP atria (RAP, red line) in presence of PCM. Panels B and C: Mean Mitochondrial $\Delta \Psi_{m}$ obtained in absence (Panel B) or presence of a saturating concentration of ADP (Panel C). Data are presented as means \pm SEM. $n=5$ to 7 independent mitochondrial isolation.

such phenotype was independent of either the mitochondrial energetic status or the respiratory substrates used (figures $5 \mathrm{C}$ and $\mathrm{D}$ ). Surprisingly, the comparison of control and RAP LV mitochondria also showed a clear trend towards a decreased $\mathrm{H}_{2} \mathrm{O}_{2}$ emission following tachypacing. Nonetheless, in most of the experimental conditions, the statistical analysis did not show significant differences between control and RAP LV mitochondria. The use of the complex III inhibitor antimycin A to stimulate mitochondrial $\mathrm{H}_{2} \mathrm{O}_{2}$ emission showed that RAP LA mitochondria produced less $\mathrm{H}_{2} \mathrm{O}_{2}$ and / or detoxifying $\mathrm{H}_{2} \mathrm{O}_{2}$ more efficiently than control $L A$ mitochondria (figure $5 \mathrm{D}$ ). In presence of Antimycin A, the LV RAP mitochondria showed no significant differences in comparison to control LV mitochondria. 
A
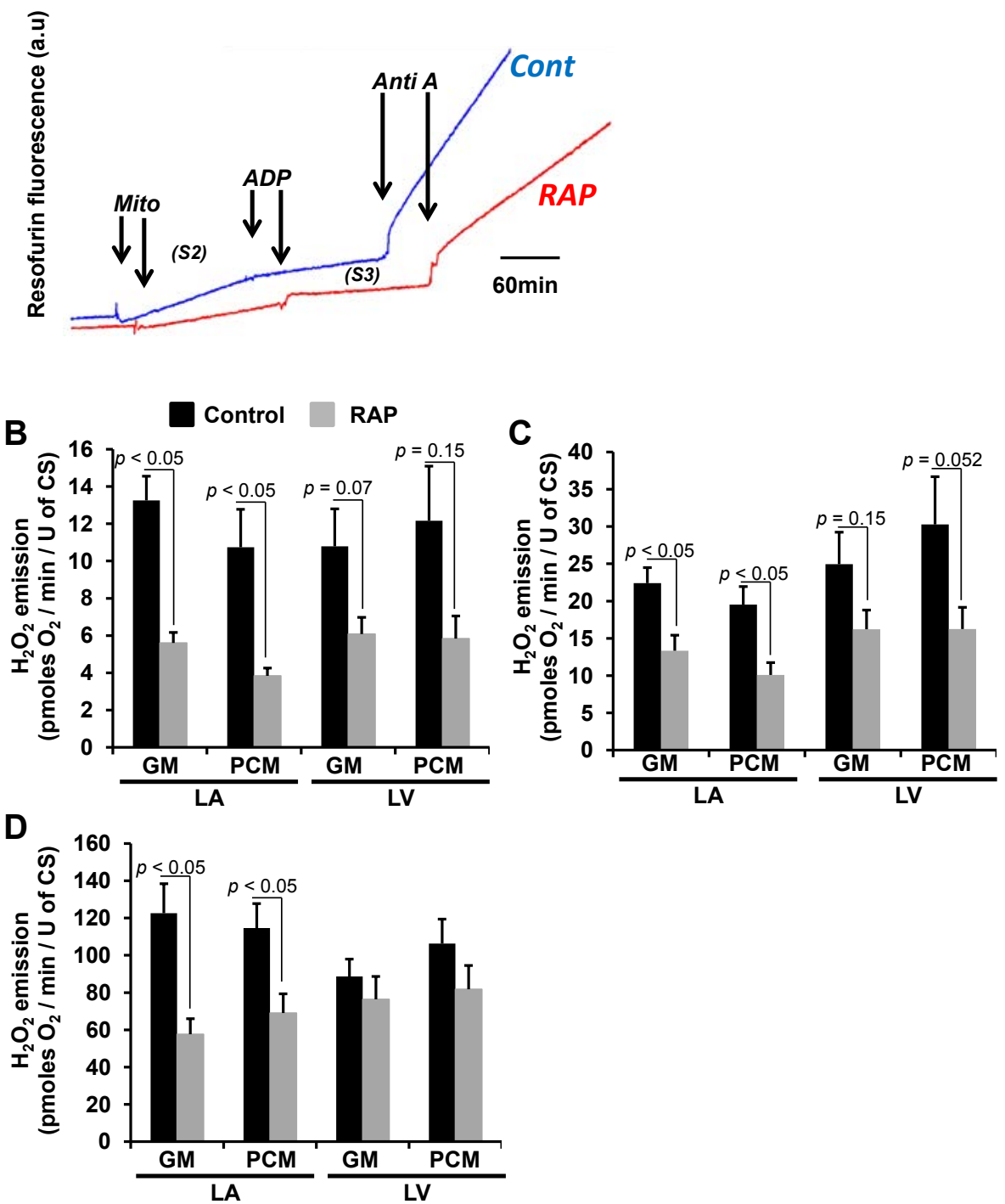

Figure 5. Effect of RAP on mitochondrial $\mathrm{H}_{2} \mathrm{O}_{2}$ emission

Mitochondria were isolated and their $\mathrm{H}_{2} \mathrm{O}_{2}$ emission was assessed in the control and RAP atria and ventricle. $\mathrm{H}_{2} \mathrm{O}_{2}$ emission was measured in each group in presence of glutamate + malate (GM) or in presence of palmitoyl-carnitine + malate (PCM). Panel A: Typical recordings of mitochondrial $\mathrm{H}_{2} \mathrm{O}_{2}$ emission obtained in the Cont atria (Cont blue line) and RAP atria (RAP, red line) in presence of $G M$. Panels B and C: Mean Mitochondrial $\Delta \Psi_{m}$ obtained in presence (Panel B) or absence of a saturating concentration of ADP (Panel C). Panel D: Mitochondria were incubated with the complex III inhibitor Antimycin A. Data are presented as means $\pm S E M . n=5$ to 7 independent mitochondrial isolation. 


\section{Alteration of mitochondrial calcium handling}

Because AF is characterized by cytosolic $\mathrm{Ca}^{2+}$ overload, we investigated the velocity at which mitochondria take up $\mathrm{Ca}^{2+}$ as well as their maximal $\mathrm{Ca}^{2+}$ retention capacity $(C R C)$. A CRC assay was used to evaluate the quantity of $\mathrm{Ca}^{2+}$ leading to mPTP opening and typical traces of such assay are shown in figures $6 \mathrm{~A}$ and $\mathrm{B}$.

Mitochondrial $\mathrm{Ca}^{2+}$ uptake velocity was assessed by integrating the area under each peak obtained following a $\mathrm{Ca}^{2+}$ addition during the $\mathrm{CRC}$ assay (figure $6 \mathrm{~A}$ ). The area obtained was calibrated and normalized for the integration time in order to express it as $\mu \mathrm{mol} / \mathrm{L}$ of total $\mathrm{Ca}^{2+}$ per min. Figure $6 \mathrm{C}$ shows the evolution of the mitochondrial $\mathrm{Ca}^{2+}$ uptake velocity as a function of the quantity of $\mathrm{Ca}^{2+}$ added during the $\mathrm{CRC}$ assay and expressed as $\mu \mathrm{mol} / \mathrm{L}$ of free $\mathrm{Ca}^{2+}$ per unit of citrate synthase. In this figure, each point corresponds to one $\mathrm{Ca}^{2+}$ addition, and all the runs performed in the control and RAP LA mitochondria groups oxidising $G M$ are plotted. Figures $6 \mathrm{~A}$ and $\mathrm{C}$ show that RAP LA mitochondria oxidizing GM were more efficient in taking up $\mathrm{Ca}^{2+}$.

In figure 6D, the mean mitochondrial $\mathrm{Ca}^{2+}$ uptake velocity for the LA and LV in the presence of GM and PCM are shown. Supplementary figures $1 C$ and $1 D$ show the mean mitochondrial $\mathrm{Ca}^{2+}$ uptake velocity for a quantity of $\mathrm{Ca}^{2+}$ that did not induce mPTP opening. LA RAP mitochondria showed a significant increase in their $\mathrm{Ca}^{2+}$ uptake velocity when compared to the LA control group in presence of GM. In contrast, this parameter was not affected when mitochondria were oxidizing PCM. Unexpectedly, this analysis showed that in the RAP LV mitochondria this parameter was decreased when mitochondria were incubated in presence of PCM and unaffected in presence of GM (Figure 6D).

In RAP LA mitochondria, the effect of the MPTP inhibitor cyclosporine A (CsA) was significantly impaired when compared to the control LA only when mitochondria were incubated in presence of PCM. This result was not caused by mitochondrial depletion of Cyclophilin D because in presence of GM the effect of CsA did not differ between RAP and control LA mitochondria (figure 6E). In the RAP LV mitochondria, mPTP sensitivity to $\mathrm{Ca}^{2+}$ was decreased in presence of $\mathrm{GM}$, but not $\mathrm{PCM}$, in comparison to control LV mitochondria (Figure 6F).

\section{Mitochondrial size and cardiolipin content}

Following one week of maintained AF in the pig, the mitochondrial morphology was altered. As shown in figure 7A, RAP lead to a decrease in average area per mitochondrion in the LA, but not in the LV (figure $7 B$ ). Although mitochondrial size was decreased in the LA, their total number per surface area analysed was unchanged (figure $7 C$ ). This suggests that RAP decreases the total surface area of mitochondrial membrane. This hypothesis was confirmed by the fact that the total content of cardiolipin, a lipid only found in mitochondrial membranes, showed a pronounced decrease in the RAP LA when compared to control LA (Figure 7D). In 
the $L V$, the mitochondrial size and density (figures $7 \mathrm{~B}, 7 \mathrm{C}$ and supplemental figure $2 \mathrm{~A}$ ) as well as the total cardiolipin content (figure 7D) were unaffected by one week of AF despite the absence of ventricular rhythm control. Although not statistically significant, RAP LA showed a strong trend towards an increased oxidized CL to total $\mathrm{CL}$ content ratio (figure $7 \mathrm{E}$ ). In contrast, RAP LV showed a significant decrease of this ratio. 


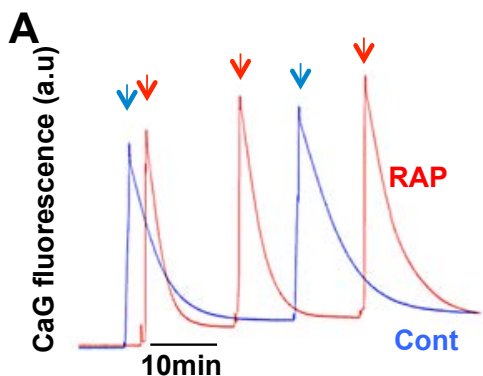

C

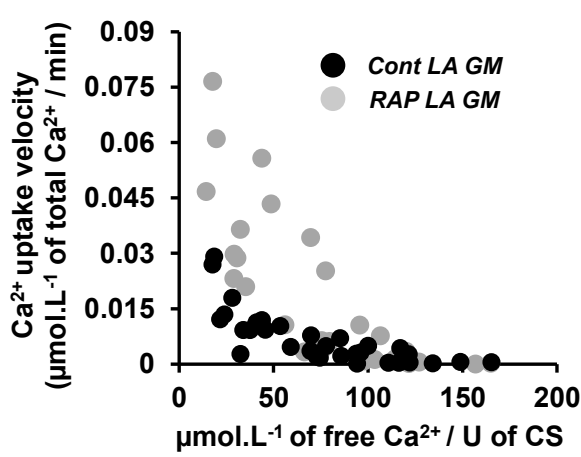

E

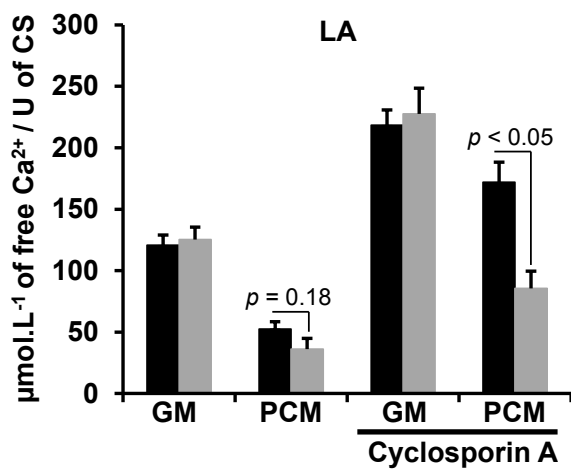

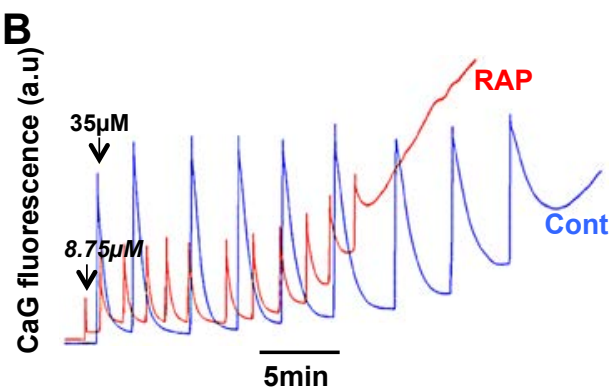

D

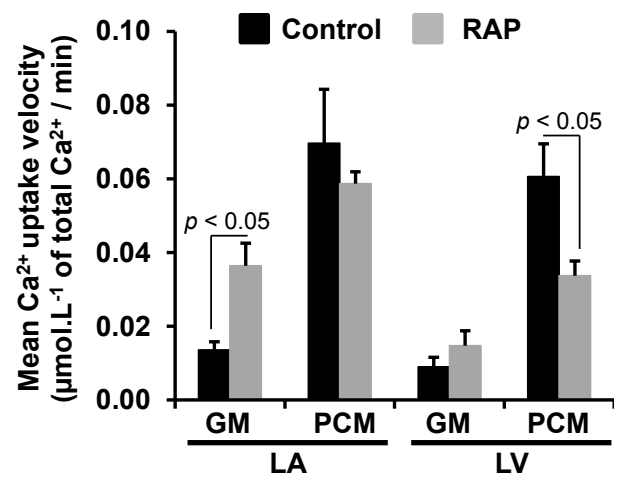

$\mathbf{F}$

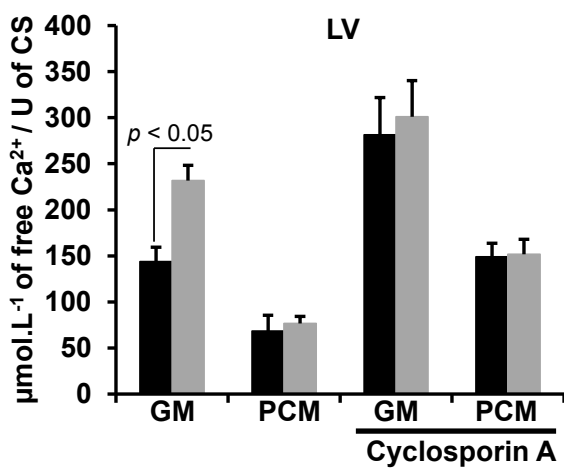

Figure 6. Effect of RAP on mitochondrial $\mathrm{Ca}^{2+}$ handling capacity Panels A and B: Typical recordings obtained during a CRC assay in the Control (Cont, blue line) and RAP (RAP, red line) in atria mitochondria in presence of GM. Panel C: Plot showing the speed of mitochondrial $\mathrm{Ca}^{2+}$ uptake during a CRC assay on control and RAP isolated atrial mitochondria respiring on glutamate + malate (GM) as a function of the free $\mathrm{Ca}^{2+}$ quantity normalized to the mitochondrial citrate synthase content Panel D: Mean mitochondrial $\mathrm{Ca}^{2+}$ uptake velocity for a low free $\mathrm{Ca}^{2+}$ quantity that does not lead to MPTP opening. Panels E and F: Mean quantity of free $\mathrm{Ca}^{2+}$ normalized to CS content leading to MPTP opening in the atria (panel E) and ventricle (panel F). Data are presented as means \pm SEM. $n=5$ to 7 independent mitochondrial isolation in the control (LA and LV) and RAP (LA + LV) groups. GM: glutamate + malate; PCM: palmitoyl-carnitine + malate. 
A

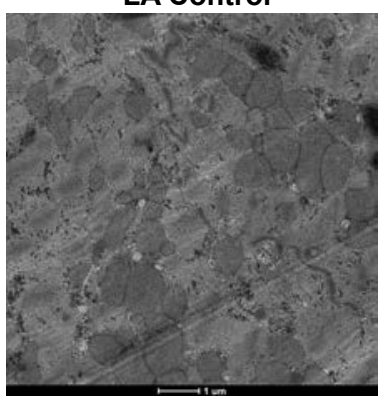

C

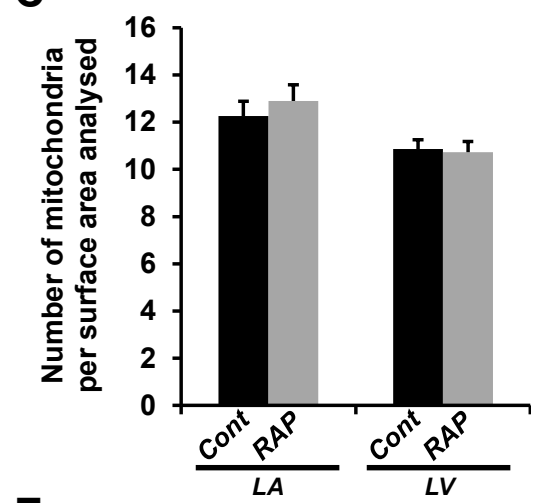

E

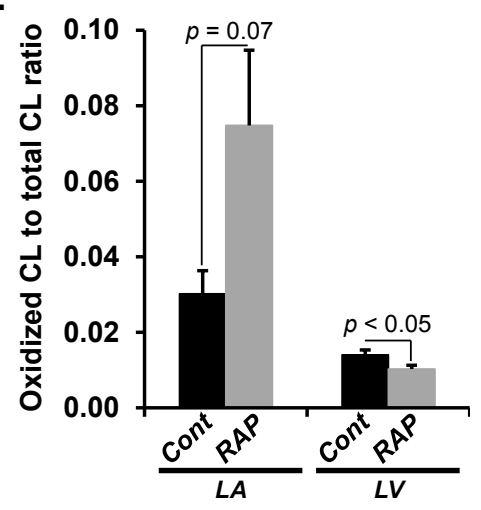

B
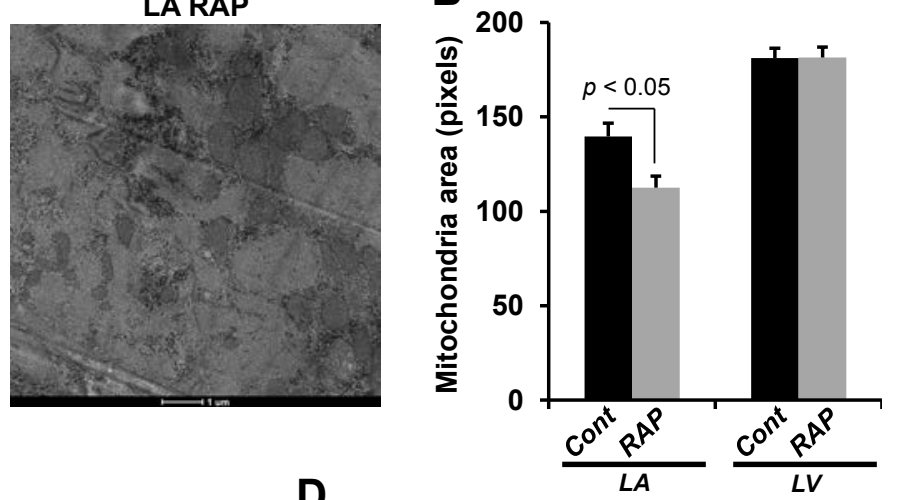

D

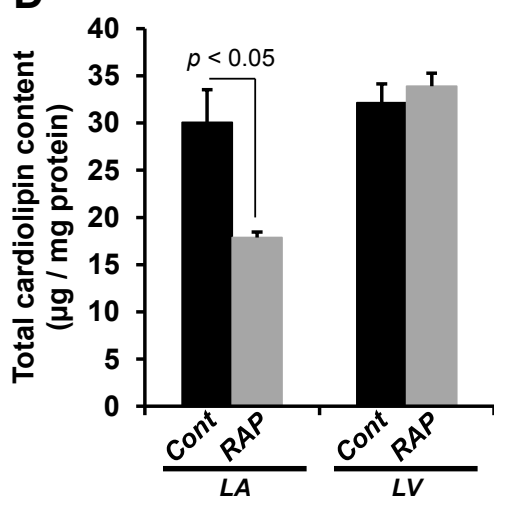

Figure 7. Effect of RAP on mitochondrial architecture

Panel A: Electron micrograph of left atrial (LA) cross-sections in the control (left panel) and RAP (right panel) groups. Panel B: Quantification of mitochondrial size. Panel C: Quantification of the total number of mitochondria per cross-section analyzed. Panel D: Total atrial and ventricular cardiolipin (CL) content assessed in the control and RAP groups. Panel E: Mean oxidized CL to total CL content in the Cont and RAP atria and ventricle. 
A

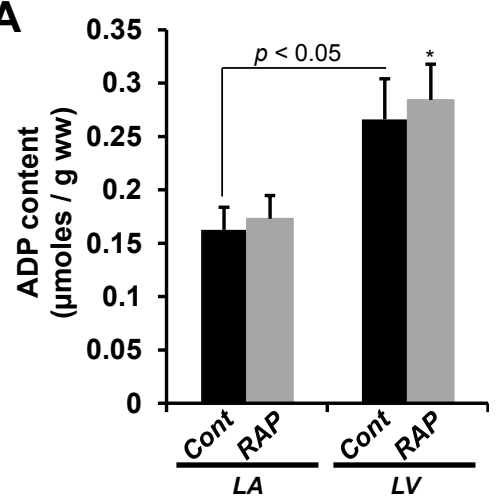

C

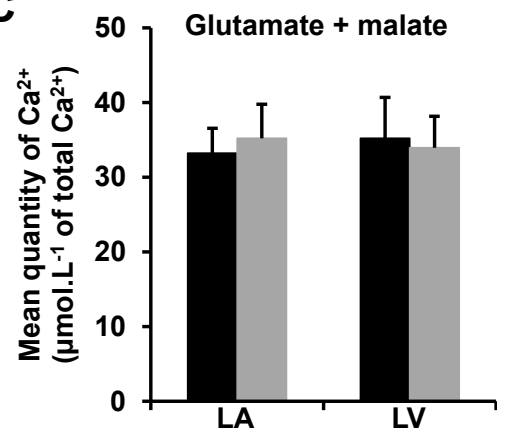

B

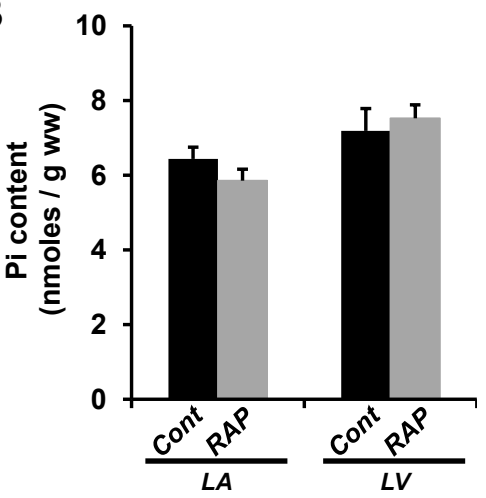

D

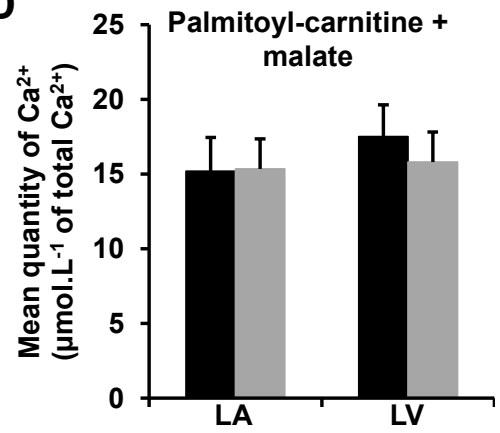

\section{Supplemental figure 1.}

Panels A and B: Pieces of LA and LV were snap-frozen in-vivo directly in the chest of the animal. Samples were then pulverized and an acidic extraction was performed to extract the energetic intermediates. Panels A and B: ADP (panel A) and inorganic phosphate (Pi, panel B) contents were assessed as described in the methods section. Panels $\mathbf{C}$ and $\mathbf{D}$ : Bar graph showing the mean $\mathrm{Ca}^{2+}$ quantity used to calculate the mitochondrial $\mathrm{Ca}^{2+}$ uptake velocity presented in the figure $6 \mathrm{D}$ of the main manuscript. 
A

LV Control

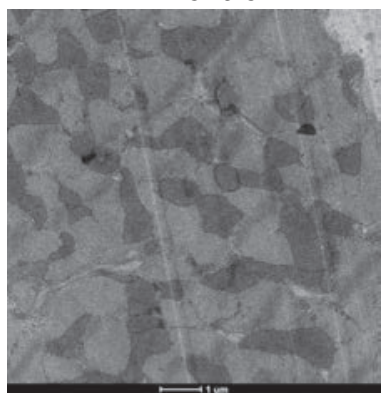

LV RAP

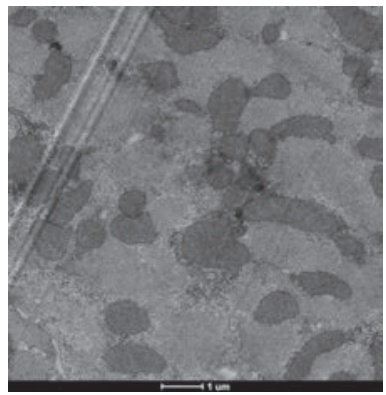

B
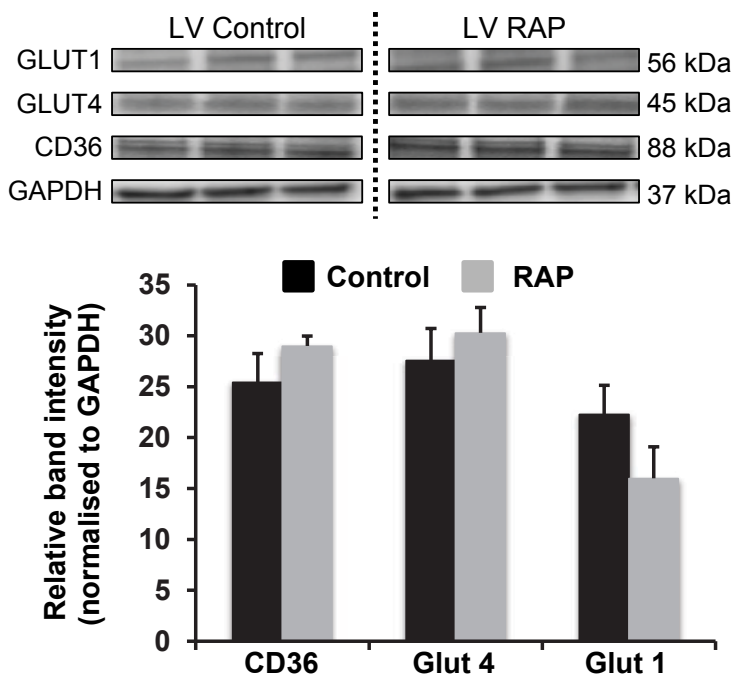

\section{Supplemental figure 2.}

Panel A: Electron micrograph of left ventricular (LA) cross-sections in the control (left panel) and RAP (right panel) groups. Panels B: Representative Western-blots of Glut 1\&4 and CD36 expression level in the control and RAP ventricles and mean data normalized to the GAPDH. 


\section{DISCUSSION}

\section{Atrial metabolic remodelling and mitochondrial alterations}

In this study, we show that in a pig model, one week of AF causes metabolic remodelling, mitochondrial alterations in structure and function as well as an impaired energetic status. AF is characterized by a metabolic reorientation leading to glycogen accumulation ${ }^{4}$. Patients with persistent AF were characterized by a pronounced use of ketone bodies to feed the tricarboxylic acid cycle and by a concomitant inhibition of glycolysis ${ }^{11}$. G-6-P is a glycolytic intermediate that is used by several metabolic pathways and is a necessary precursor for glycogen synthesis. Our results shows that 1 week of RAP is sufficient to increase the G-6-P content twofold. This accumulation was not associated with increased expression of the glucose transporters Glut-1 and -4. RAP also leads to an increased activity of the total mitochondrial hexokinase specific activity. Hexokinase is the first enzyme of the glycolytic pathway, catalyzing the phosphorylation of glucose to G-6-P. An increase of hexokinase expression would favour glucose entrapment in the atrial myocyte. We have previously shown that acute AF leads to an increase in atrial lactate production ${ }^{16}$. At present, we do not know how long this increase in lactate production persists, but increased G-6-P production in combination with reduced mitochondrial pyruvate consumption should favour G-6-P accumulation and glycogen storage.

In the RAP atria, mitochondrial oxygen consumption linked to glucose-like metabolism (i.e. glutamate + malate) was increased in phosphorylating conditions, while no changes were observed in oxygen consumption during fatty-acid metabolism measured under the same experimental conditions. In contrast, in absence of ADP, the basal respiration significantly decreased in presence of PCM only. The origin of this decrease is not known but could be related to the decrease in cardiolipin content. Indeed, in absence of ADP, mitochondrial respiration is driven by the permeability of the inner mitochondrial membrane (IMM) to protons. One week of RAP led to a $50 \%$ decrease in the cardiolipin content (figure 7D). A significant decrease of cardiolipin, which is the major lipid species found in the IMM, may affect the passive permeability to protons and thus basal mitochondrial oxygen consumption.

Unexpectedly, LA mitochondrial $\mathrm{H}_{2} \mathrm{O}_{2}$ emission in absence and presence of ADP was decreased following one week of RAP and this was independent of the respiratory substrates used. Moreover, maximal $\mathrm{H}_{2} \mathrm{O}_{2}$ emission, stimulated by inhibition of the respiratory complex III, was also lower in the RAP LA than in the control LA mitochondria. These results likely reflect an adaptive mechanism. Although not significant ( $p$ value $=0.07$ ), the oxidized cardiolipin to total cardiolipin content ratio was increased following one week of RAP when compared to control. This suggests that LA RAP mitochondria evolve in a pro-oxidant environment which is in line with the fact that $A F$ is associated to an increase of the damage linked to oxidative stress 7. Interestingly, RAP LV mitochondria showed less $\mathrm{H}_{2} \mathrm{O}_{2}$ emission as well (Figure 
5) and this was associated with lower cardiolipin oxidation. Thus, in both cardiac compartments, increased activation rates were associated to a positive adaptation towards less mitochondrial $\mathrm{H}_{2} \mathrm{O}_{2}$ emission. In absence of ADP the mitochondrial $\mathrm{H}_{2} \mathrm{O}_{2}$ emission is mainly dependent on the mitochondrial $\Delta \Psi_{m}$. Although RAP modulated the basal respiration in opposite direction in atria and ventricle in presence of $G M$, the mitochondrial $\mathrm{H}_{2} \mathrm{O}_{2}$ emission was decreased in both atria and ventricles. Thus, it will be important in the future to assess if one week of RAP is accompanied by an increased activity of mitochondrial anti-oxidant mechanisms.

It is important to note that our experimental approach to assess mitochondrial $\Delta \Psi_{\mathrm{m}}$ is qualitative and not quantitative. Consequently, the lack of difference between the RAP and control mitochondria in absence of ADP does not mean that the mitochondrial $\Delta \Psi_{\mathrm{m}}$ absolute values were identical between these groups. To assess the impact of RAP on the regulation of mitochondrial $\Delta \Psi_{m}$ a different methodological approach (i.e. use of a TPP ${ }^{+}$electrode for instance) would be required. Such approach would be necessary to evaluate if some of the mitochondrial alterations in response to RAP correlate to a change in mitochondrial $\Delta \Psi_{\mathrm{m}}$ for given bioenergetic status. Nonetheless, our experimental approach allowed us to show that in presence of fatty-acids, RAP LA mitochondria were more depolarized during phosphorylating conditions than control mitochondria. Interestingly, the oxygen consumption measured at the same condition was not altered, suggesting an alteration of the proton-to-electron ratio during PCM oxidation.

In presence of GM, RAP LA mitochondrial $\mathrm{Ca}^{2+}$ uptake velocity was increased twofold in presence of $\mathrm{GM}$. At present, we cannot determine the mechanistic origin of this phenomenon. Potentially, a faster mitochondrial $\mathrm{Ca}^{2+}$ uptake would be a useful adaptation limiting the cytosolic $\mathrm{Ca}^{2+}$ overload caused by increased atrial rates. In future experiments, the development of a simultaneous measurement of $\mathrm{Ca}^{2+}$ uptake velocity and mitochondrial $\Delta \Psi_{\mathrm{m}}$ would be a necessary step to better understand the origin of these differences. One week of RAP leads to MPTP sensitization to $\mathrm{Ca}^{2+}$ in presence of CsA only when RAP LA mitochondria oxidized fatty-acids. mPTP has been proposed to be a voltage gated channel ${ }^{22}$, thus the increased mPTP sensitivity to $\mathrm{Ca}^{2+}$ might be secondary to more depolarized mitochondria in presence of PCM. Moreover, the difference in the effect of CSA was not observed in the RAP LA mitochondria oxidizing GM. Consequently, the decreased effect of CsA observed in the RAP group in the presence of fatty-acids is not secondary to a Cyclophilin D depletion. In patients with metabolic syndrome, a downregulation of $\beta$-oxidation enzymes has been shown to be significantly correlated to the development of postoperative atrial fibrillation. ${ }^{15}$ The same authors have proposed that POAF might be influenced by MPTP opening. 


\section{Atrial energetic status and mitochondrial structural remodelling}

The atrial energetic status has been investigated on samples directly frozen in the chest of the animal. Such approach was critical to avoid ischemia during harvesting of the samples; even a few seconds of ischemia is sufficient to promote PCr hydrolysis and concomitant increase in inorganic phosphate and AMP. Our results show that after 1 week of RAP, ATP and PCr content decrease without affecting the total content of inorganic phosphate or ADP. We have previously shown in a goat model of rapid atrial tachypacing that 1 week was sufficient to decrease the $\mathrm{PCr}$ content ${ }^{8}$. So far it is not known whether this new energetic state is the consequence of an alteration of ATP synthesis or to an increased energetic demand caused by rapid atrial rates. The decreased content in cardiolipin may reflect a reduced IMM area, corresponding to a reduced capacity of mitochondrial ATP and PCr synthesis and/or export. Indeed, cardiolipin has been shown to be critical for the function of several mitochondrial proteins among which the mitochondrial creatine kinase ${ }^{19}$. Under the assumption that after 1 week of RAP the total pool of creatine is identical in the control and RAP groups, the PCr to Pi ratio gives an indication of the phosphorylation potential $(\triangle G \mathrm{p})$. As we show, the PCr to Pi ratio was significantly decreased in RAP atria when compared to control atria. We can therefore conclude that 1 week of RAP leads to a significant deterioration in the atrial energetic status. In the RAP atria, the depressed $\triangle \mathrm{Gp}$ did not lead to phosphorylation of AMPK. AMPK is a crucial cellular energy sensor that is activated when the cellular energetic status is falling. It is important to note that the $\beta$ and $\gamma$ subunits of the AMPK are regulated by AMP and ADP ${ }^{23}$. Although we showed a decrease in the ATP to ADP ratio in the RAP atria, this was caused by a decreased level of ATP without change in total ADP. This observation could potentially explain why RAP does not lead to AMPK activation.

LA RAP mitochondria in presence of $G M$ were able to take up $\mathrm{Ca}^{2+}$ more rapidly than LA control mitochondria. It is known that RAP/ AF leads to intercellular $\mathrm{Ca}^{2+}$ overload ${ }^{24}$, which would also lead to mitochondrial $\mathrm{Ca}^{2+}$ accumulation ${ }^{10}$. This may contribute to decreased ATP synthesis through dissipation of $\Delta \Psi_{\mathrm{m}}$. To avoid mitochondrial $\mathrm{Ca}^{2+}$ overload and mPTP opening, $\mathrm{Ca}^{2+}$ is extruded through the $\mathrm{Na}^{+} / \mathrm{Ca}^{2+}$ exchanger and the accumulating $\mathrm{Na}^{+}$is extruded through the $\mathrm{Na}^{+} / \mathrm{H}^{+}$exchanger ${ }^{25},{ }^{26}$. Consequently, a cytosolic $\mathrm{Ca}^{2+}$ accumulation might decrease ATP synthesis through $\Delta \Psi_{\mathrm{m}}$ consumption by a futile $\mathrm{Ca}^{2+}$ cycling phenomenon. Interestingly, Ausma and colleagues showed that RAP in a goat model decreased the PCr content without affecting the activity of several mitochondrial enzymes ${ }^{8}$. Thus, an increased energy consumption in combination to the development of futile mitochondrial $\mathrm{Ca}^{2+}$ cycling might explain the decreased $\Delta G p$ as a results of RAP.

We also demonstrate that one week of RAP is sufficient to alter mitochondrial morphology in our pig model. The mechanisms causing these structural changes are not clear. Nonetheless, RAP atria showed a 50\% decrease in their cardiolipin 
content. Cardiolipin participates to the formation of contact sites through their electrostatic interaction with the positively charged residues of the octameric mitochondrial creatine kinase $(\mathrm{mtCK})^{27}$. This enzyme is responsible for the synthesis of PCr from the mitochondrial ATP and is thought to be at the heart of contact sites. Thus, an alteration in the cardiolipin content might have an impact on mtCK function, formation of contact sites and consequently $\mathrm{PCr}$ export to the cytosol. It has been shown that a decrease in the expression level of mtCK can profoundly alter mitochondrial morphology ${ }^{28}$. It would be interesting to investigate whether decreased cardiolipin content as a result of AF is sufficient to destabilize mtCK octamers and is responsible for alterations in mitochondrial morphology.

\section{Impact of RAP on ventricular mitochondrial function}

In our experimental model, the ventricular rhythm was not controlled. In earlier studies in dogs and pigs, RAP significantly increased ventricular rate ${ }^{29,}{ }^{30}$. It is interesting to note that in our model, the increased ventricular rate as a result of RAP only had an effect on mitochondrial function, but did not affect mitochondrial structure or $\mathrm{CL}$ content. With respect to mitochondrial function, the changes comprised a modulation of the oxygen consumption, decreased $\mathrm{H}_{2} \mathrm{O}_{2}$ emission and altered mitochondrial $\mathrm{Ca}^{2+}$ handling properties. RAP ventricular mitochondria were less efficient in taking up $\mathrm{Ca}^{2+}$ in presence of PCM and their ADP-stimulated respiration was increased in presence of GM. Interestingly, mPTP sensitivity to $\mathrm{Ca}^{2+}$ was decreased in presence of GM while no changes were observed in presence of PCM. At this stage, we can only hypothesize that these mitochondrial adaptations might result from the increased cytosolic $\mathrm{Ca}^{2+}$ turnover secondary to the increased ventricular rate during RAP. The ventricular mitochondrial phenotype might be the results of a positive adaptive mechanism. It is known that in the pig, a prolonged period of RAP leads to heart failure ${ }^{31}$. Thus, in this model it will be important to assess the evolution of ventricular mitochondrial function to establish whether heart failure and $\Delta G \mathrm{p}$ depression are preceded by an alteration of mitochondrial function.

\section{Limitations}

We recognize that the use of isolated mitochondria has important limitations and that great care must be taken to translate our results to the intact heart or animal. It will be necessary in future studies to further develop our experimental approach on more integrated system (i.e. isolated cells). Nonetheless, our experimental approach allowed us to demonstrate that in the pig, RAP leads to metabolic and mitochondrial alterations in the atria as well as in the ventricle. Future studies will have to be performed to explain the exact mechanisms by which early mitochondrial remodelling contributes to the energetic impairment caused by AF. 


\section{CONCLUSIONS}

Our results show that, after a short period of induced AF by RAP in the pig, mitochondrial architecture and function are affected, leading to an alteration of the bioenergetic status of the atria. AF seems to alter mitochondrial polarization in presence of fatty acids under phosphorylating conditions. The disturbance of $\Delta \Psi_{\mathrm{m}}$ regulation might be responsible for the increased mPTP sensitivity to $\mathrm{Ca}^{2+}$ observed in presence of PCM and CSA. This phenomenon was paralleled by an increased mitochondrial $\mathrm{Ca}^{2+}$ uptake velocity in presence of $\mathrm{GM}$. We hypothesize that increased mitochondrial $\mathrm{Ca}^{2+}$ cycling, in combination with the decreased cardiolipin content may contribute to the alteration of mitochondrial morphology and the impairment of the cellular energetic status. 


\section{REFERENCES}

1. Wijffels MC, Kirchhof CJ, Dorland R, Allessie MA. Atrial fibrillation begets atrial fibrillation. A study in awake chronically instrumented goats. Circulation 1995;92:1954-1968.

2. Schotten U, Verheule S, Kirchhof P, Goette A. Pathophysiological mechanisms of atrial fibrillation: a translational appraisal. Physiological reviews 2011;91:265-325.

3. Schotten U, de Haan S, Neuberger HR, Eijsbouts S, Blaauw Y, Tieleman R, Allessie M. Loss of atrial contractility is primary cause of atrial dilatation during first days of atrial fibrillation. American journal of physiology Heart and circulatory physiology 2004;287:H2324-2331.

4. Ausma J, Wijffels M, Thone F, Wouters L, Allessie M, Borgers M. Structural changes of atrial myocardium due to sustained atrial fibrillation in the goat. Circulation 1997;96:3157-3163.

5. Greiser M, Neuberger HR, Harks E, El-Armouche A, Boknik P, de Haan S, Verheyen F, Verheule S, Schmitz W, Ravens U, Nattel S, Allessie MA, Dobrev D, Schotten U. Distinct contractile and molecular differences between two goat models of atrial dysfunction: AV block-induced atrial dilatation and atrial fibrillation. Journal of molecular and cellular cardiology 2009;46:385-394.

6. Ausma J, Wijffels M, van Eys G, Koide M, Ramaekers F, Allessie M, Borgers M. Dedifferentiation of atrial cardiomyocytes as a result of chronic atrial fibrillation. The American journal of pathology 1997;151:985-997.

7. Wolke C, Bukowska A, Goette A, Lendeckel U. Redox control of cardiac remodeling in atrial fibrillation. Biochimica et biophysica acta 2015;1850:1555-1565.

8. Ausma J, Coumans WA, Duimel H, Van der Vusse GJ, Allessie MA, Borgers M. Atrial high energy phosphate content and mitochondrial enzyme activity during chronic atrial fibrillation. Cardiovascular research 2000;47:788-796.

9. Ausma J, Litjens N, Lenders MH, Duimel H, Mast F, Wouters L, Ramaekers F, Allessie M, Borgers $\mathrm{M}$. Time course of atrial fibrillation-induced cellular structural remodeling in atria of the goat. Journal of molecular and cellular cardiology 2001;33:2083-2094.

10. Ausma J, Dispersyn GD, Duimel H, Thone F, Ver Donck L, Allessie MA, Borgers M. Changes in ultrastructural calcium distribution in goat atria during atrial fibrillation. Journal of molecular and cellular cardiology 2000;32:355-364.

11. Mayr M, Yusuf S, Weir G, Chung YL, Mayr U, Yin X, Ladroue C, Madhu B, Roberts N, De Souza A, Fredericks S, Stubbs M, Griffiths JR, Jahangiri M, Xu Q, Camm AJ. Combined metabolomic and proteomic analysis of human atrial fibrillation. Journal of the American College of Cardiology 2008;51:585-594.

12. Halestrap AP, Pereira GC, Pasdois P. The role of hexokinase in cardioprotection - mechanism and potential for translation. British journal of pharmacology 2015;172:2085-2100. 
13. Halestrap AP. Mitochondrial calcium in health and disease. Biochimica et biophysica acta 2009;1787:1289-1290.

14. Weber KT, Sun Y, Bhattacharya SK, Ahokas RA, Gerling IC. Myofibroblast-mediated mechanisms of pathological remodelling of the heart. Nature reviews Cardiology 2013;10:1526.

15. Montaigne D, Marechal X, Lefebvre P, Modine T, Fayad G, Dehondt H, Hurt C, Coisne A, Koussa M, Remy-Jouet I, Zerimech F, Boulanger E, Lacroix D, Staels B, Neviere R. Mitochondrial dysfunction as an arrhythmogenic substrate: a translational proof-ofconcept study in patients with metabolic syndrome in whom post-operative atrial fibrillation develops. Journal of the American College of Cardiology 2013;62:1466-1473.

16. van Bragt KA, Nasrallah HM, Kuiper M, Luiken JJ, Schotten U, Verheule S. Atrial supplydemand balance in healthy adult pigs: coronary blood flow, oxygen extraction, and lactate production during acute atrial fibrillation. Cardiovascular research 2014;101:9-19.

17. Pasdois P, Parker JE, Griffiths EJ, Halestrap AP. The role of oxidized cytochrome c in regulating mitochondrial reactive oxygen species production and its perturbation in ischaemia. The Biochemical journal 2011;436:493-505.

18. Pasdois P, Parker JE, Halestrap AP. Extent of mitochondrial hexokinase II dissociation during ischemia correlates with mitochondrial cytochrome c release, reactive oxygen species production, and infarct size on reperfusion. Journal of the American Heart Association 2013;2:e005645.

19. Schlame M, Shanske S, Doty S, Konig T, Sculco T, DiMauro S, Blanck TJ. Microanalysis of cardiolipin in small biopsies including skeletal muscle from patients with mitochondrial disease. Journal of lipid research 1999;40:1585-1592.

20. Valianpour F, Wanders RJ, Barth PG, Overmars H, van Gennip AH. Quantitative and compositional study of cardiolipin in platelets by electrospray ionization mass spectrometry: application for the identification of Barth syndrome patients. Clinical chemistry 2002;48:1390-1397.

21. Liu Y, Geng J, Liu Y, Li Y, Shen J, Xiao X, Sheng L, Yang B, Cheng C, Li W. betaz-adrenoceptor mediates metabolic protein remodeling in a rabbit model of tachypacing-induced atrial fibrillation. Cellular physiology and biochemistry : international journal of experimental cellular physiology, biochemistry, and pharmacology 2013;32:1631-1642.

22. Bernardi P, Broekemeier KM, Pfeiffer DR. Recent progress on regulation of the mitochondrial permeability transition pore; a cyclosporin-sensitive pore in the inner mitochondrial membrane. Journal of bioenergetics and biomembranes 1994;26:509-517.

23. Carling D, Thornton C, Woods A, Sanders MJ. AMP-activated protein kinase: new regulation, new roles? The Biochemical journal 2012;445:11-27. 
24. Goette A, Honeycutt C, Langberg JJ. Electrical remodeling in atrial fibrillation. Time course and mechanisms. Circulation 1996;94:2968-2974.

25. Halestrap AP. Calcium-dependent opening of a non-specific pore in the mitochondrial inner membrane is inhibited at pH values below 7. Implications for the protective effect of low pH against chemical and hypoxic cell damage. The Biochemical journal 1991;278 ( Pt 3):715719 .

26. Allen DG, Orchard CH. Myocardial contractile function during ischemia and hypoxia. Circulation research 1987;60:153-168.

27. Schlame M, Augustin W. Association of creatine kinase with rat heart mitochondria: high and low affinity binding sites and the involvement of phospholipids. Biomedica biochimica acta $1985 ; 44: 1083-1088$.

28. Lenz H, Schmidt M, Welge V, Kueper T, Schlattner U, Wallimann T, Elsasser HP, Wittern KP, Wenck H, Staeb F, Blatt T. Inhibition of cytosolic and mitochondrial creatine kinase by siRNA in HaCaT- and HeLaS3-cells affects cell viability and mitochondrial morphology. Molecular and cellular biochemistry 2007;306:153-162.

29. Morillo CA, Klein GJ, Jones DL, Guiraudon CM. Chronic rapid atrial pacing. Structural, functional, and electrophysiological characteristics of a new model of sustained atrial fibrillation. Circulation 1995;91:1588-1595.

30. Giddins NG, Duncan K, Persad R, Gilchrist JS, Pierce GN. Effects of chronic, rapid right atrial pacing on cardiac hemodynamics and myofibrillar ATPase activity in piglets. The Canadian journal of cardiology 2002;18:157-161.

31. McElmurray JH, Mukherjee R, Patterson TM, Goldberg A, King MK, Hendrick JW, Gay DM, Clair MJ, Jolly JR, Spinale FG. Comparison of amlodipine or nifedipine treatment with developing congestive heart failure: effects on myocyte contractility. Journal of cardiac failure 2001;7:158-164. 
General Discussion

Hussein M Nasrallah

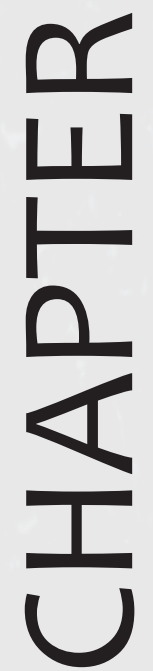




\section{GENERAL DISCUSSION}

\section{Atrial oxygen supply and demand}

Atrial fibrillation (AF), the most common sustained arrhythmia, is characterized by rapid and irregular activation of the atria. AF is self-perpetuating because it leads to electrical and structural remodeling that makes the arrhythmia more stable. Whereas electrical remodeling is a fast process (1-2 days), structural remodeling develops much more slowly (months to years) ${ }^{1,2}$. Structural remodeling leads to loss of side-to-side connections between myocyte bundles and thereby to an increased complexity of fibrillatory conduction ${ }^{3,4}$. However, the exact mechanisms that are responsible for structural remodeling are not clearly understood.

As in any other tissue, blood supply by the atrial coronaries is regulated to match the energy/ oxygen demand ${ }^{5}$. In the heart, it is particularly important that even under increased demand, metabolism remains aerobic, to avoid acidification and muscle fatigue that would be detrimental to cardiac pump function. Therefore the balance between oxygen supply and demand has to be carefully guarded (figure 1$)^{6}$. Some important determinants of myocardial oxygen demand are heart rate ${ }^{7}$, myocardial shortening ${ }^{8}$, myocardial wall tension ${ }^{9}$ and contractility ${ }^{10}$. During AF, the rate of electrical and contractile activity increases 5-6 fold, which is likely to increase atrial energy and oxygen expenditure considerably. If this increase in demand cannot be met, a state of supply/demand ischemia ensues. We have previously shown that in pigs, acute AF indeed leads to acute supply/demand ischemia, as evidenced by atrial lactate production during $\mathrm{AF}^{11}$ and reactive hyperemia after a short $\mathrm{AF}$ episode ${ }^{5}$. This subtle form of ischemia may persist in the important first weeks of AF pathogenesis. Goats with one week of AF have shown a decrease in creatine phosphate $(\mathrm{PCr})^{12}$ and increased expression of HIF1-a ${ }^{13}$. Moreover, the alterations in atrial ultrastructure were similar to changes observed in hibernating ventricular myocardium due to low flow ischemia ${ }^{14}$. Patients with AF exhibited increased atrial expression of hypoxic (HIF1-a) and angiogenic (VEGF) markers implying a state of atrial oxygen shortage ${ }^{15}$, ${ }^{16}$.

The atrial myocardium may respond in two ways to restore the supply/demand balance: by increasing supply and by decreasing demand. Increasing supply on the longer term may be effected by arteriogenesis and angiogenesis, which is the normal response to activation of the HIF1-a/ VEGF pathway ${ }^{17}$.

Demand may be decreased by decreasing cellular ion fluxes and by decreasing contractility. Indeed, electrical and contractile remodeling may be interpreted to effectuate this. In addition, metabolism may shift from fatty acid oxidation to glucose oxidation, which is more oxygen-efficient (but not more substrate-efficient) ${ }^{18}$. 
In this thesis, we have assessed both metabolic and vascular remodeling in pig models of AF. We choose pigs as a model because of the similarity in coronary vascular anatomy and regulation to those in humans ${ }^{6,19}$.

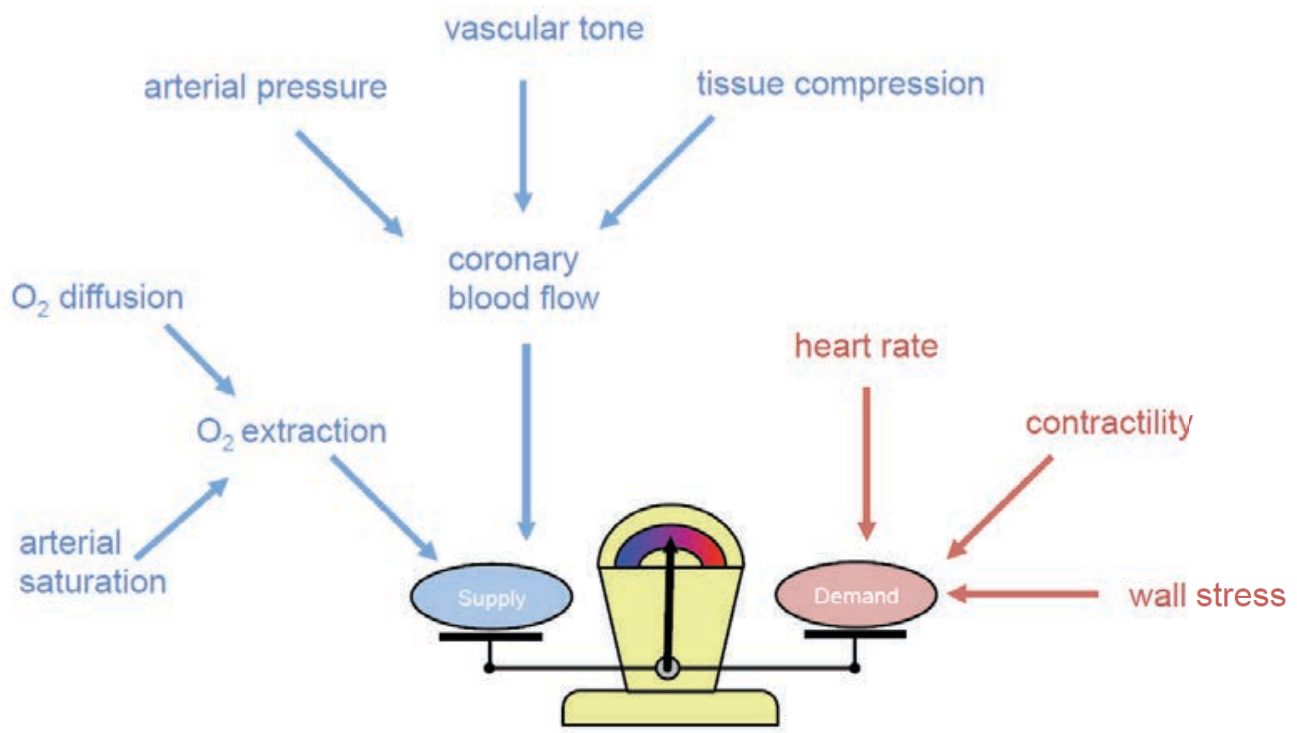

Figure 1. Schematic representation depicting the main determinants of myocardial oxygen supply demand balance

\section{Metabolic remodeling}

The energy required to perform the required electrical and mechanical work is provided as chemical energy in the form of ATP ${ }^{20}$. To maintain levels of ATP production and utilization of ATP have to be balanced. From the total amount of ATP produced, 60 to $70 \%$ is used for contractility and remaining 30 to $40 \%$ for ion transport ${ }^{21}$. A deficiency in the levels of ATP directly triggers a decline in cardiac contractile performance ${ }^{20}$. In the ventricle, the main source of ATP production is the oxidation of fatty acids (70 to $90 \%$ of the ATP generation). Glucose and lactate oxidation (and oxidation of small amounts of ketone bodies and amino acids) are responsible for generation of the remaining 10 to $30 \%{ }^{20,22}$.

In a pig model, we have studied the effect of 1 week of AF on metabolite levels and on mitochondrial function and structure. We observed a decline in both PCr/Pi and ATP/ $A P D$ ratios in the $L A$ as a result of $A F$. The latter indicates that the degree of metabolic compromise is larger than in the goat model after 1 week of $A F^{12}$. The observed increase in glucose-6-phosphate and glycogen accumulation suggest a shift from fatty acid to glucose utilization, but this is not supported by corresponding shift in the expression of glucose (Glut1 and -4) and fatty acid (CD36) transporters. Isolated 
mitochondria in many respects are fully functional after 1 week of AF, with efficient respiration and increased respiratory control. Indeed, the decreased mitochondrial $\mathrm{H}_{2} \mathrm{O}_{2}$ production and increased calcium buffering velocity suggest adaptive changes in response to oxidative stress and calcium overload. However, as a result of AF, mitochondria had a depolarized membrane potential during fatty acid oxidation and the mitochondrial permeability transition pore was more sensitive to calcium. Based on our current data, the decrease in ATP and PCr levels may be best explained by the significant reduction in mitochondrial size and total cardiolipin (CL) content in the of AF pigs. These changes are likely to reflect a decrease in the total area of inner mitochondrial membrane (of which CLs are a specific constituent), and thus of the area available for the ATP synthesizing machinery in atrial myocytes.

\section{Vascular remodeling}

Vascular tone is determined by the tissue levels of vasodilators and vasoconstrictors, as well as the responsiveness of arterioles to these substances ${ }^{6}{ }^{23}$. We have studied the effect of AF on the latter in isolated atrial small muscular arteries (SMA, average size $\approx 450 \mu \mathrm{m})$. In hypertension, the increased vascular myogenic response is associated with hypertrophy of vascular smooth muscle cells ${ }^{24-26}$. By contrast, we have observed an increase in the response to receptor-dependent and -independent vasoconstrictors as a result of AF, but without smooth muscle hypertrophy. The functional consequences of this phenomenon in vivo are unclear, but it might lead to a shift towards increased vascular tone.

AF has been linked with systemic endothelial dysfunction in patients ${ }^{27,28}$. Endothelial dysfunction is evidenced by a blunted response to vasodilators such as bradykinin(BK) ${ }^{29}$. If this also occurs in arterioles in the atria itself, it would further limit the capacity of the atrial coronary vasculature to respond to fluctuations in demand. However, in isolated arterioles, we observed an increased sensitivity to BK. This endothelial 'hyperfunction' has also been observed in the heart as a result of exercise and in response to a remote, small infarct ${ }^{30,31}$. As such it may reflect an adaptive response at this early phase of AF pathogenesis that would be consistent with an increased eNOS activity ${ }^{31,32}$. Although the total eNOS level was unchanged after 1 week of AF, increased eNOS activity may still result from increased eNOS phosphorylation. In the goat model of AF, become 'uncoupled' at later stages (month) of AF ${ }^{33}$, and it would certainly be interesting to assess isolated atrial arterioles at the later stage.

In our pig model, the hypoxia marker HIF1-a was increased after one week of AF. After 5 weeks of AF, both HIF1-a and the angiogenesis market VEGF were increased. Normally, activation of HIF1-a/ VEGF signaling should lead to angiogenesis (formation of new capillaries) and, to a lesser extent, arteriogenesis (formation of new arterioles) 34,35 . However, we did not observe any change in arteriolar density and a pronounced decrease in capillary density. In the kidney, similar capillary rarefaction 
following a phase of ischemia has been linked to a high Ang-2/Ang-1 ratio, that would destabilize newly formed capillaries ${ }^{36-38}$. From our results, this mechanism cannot explain the capillary rarefaction we have observed in the atrium. Atrial capillary rarefaction represents one of the aspects of vascular remodeling that would restrict the capacity of the atria to accommodate fluctuations in energy/ oxygen demand.

In addition to the effect of AF itself, we studied vascular structure and function in a pig model of CHF, which is a strong, independent risk factor for AF in patients 39,40 . In a canine model, Nattel and coworkers have shown that CHF causes a pronounced increase in atrial fibrosis, which likely to form obstacles to conduction and thus increase AF stability ${ }^{40-42}$.

We initially hypothesized that CHF would lead to vascular dysfunction and remodeling of vascular structure that would make the atria more liable to supply/ demand ischemia. Our results show that, also in pigs, CHF leads to an increased stability of AF. However, unlike the canine model, the degree of myocardial fibrosis was not increased. Pigs with CHF did show a marked increase in atrial chamber size as well as myocyte size, which are also known to contribute to AF stability ${ }^{43-45}$. Atrial coronary flow reserve in the LA was decreased in CHF pigs. Analysis of vascular structure demonstrated a lower capillary density and increased perivascular fibrosis. Based on studies in the ventricle, the lower LA coronary flow reserve may indeed by explained by these structural changes ${ }^{46-48}$.

To investigate whether CHF atria are more vulnerable to supply/ demand ischemia, the response to atrial pacing and AF was assessed. The response of the atrial coronary conductance to both interventions was blunted, but (in a separate series of experiments) we determined that this did not lead to excess lactate production in the CHF group. Rather, the lack of increase in atrial supply seemed to be linked to a pronounced decrease in demand in CHF atria. Whereas the active work performed by the atria increased with pacing in normal pigs, atrial contractility was virtually absent in CHF pigs. In a dog model of CHF, the levels of ATP and PCr were strongly decreased ${ }^{49}$. In our pig model, we cannot determine whether this loss of atrial contractility is caused by a deficit in atrial bioenergetics during the development of CHF.

Eventually, many CHF patients develop AF, which is detrimental for ventricular pump function due to the rapid and irregular ventricular rate ${ }^{50,51}$. However, the loss of atrial contractility we have observed would already lead to a deterioration of overall pump function and exercise tolerance before the onset of AF 5253 54.

\section{Future Perspectives}

One of the major goals of this thesis was to determine whether AF and CHF (as a risk factor for AF) lead to atrial vascular and metabolic remodeling. The data in this 


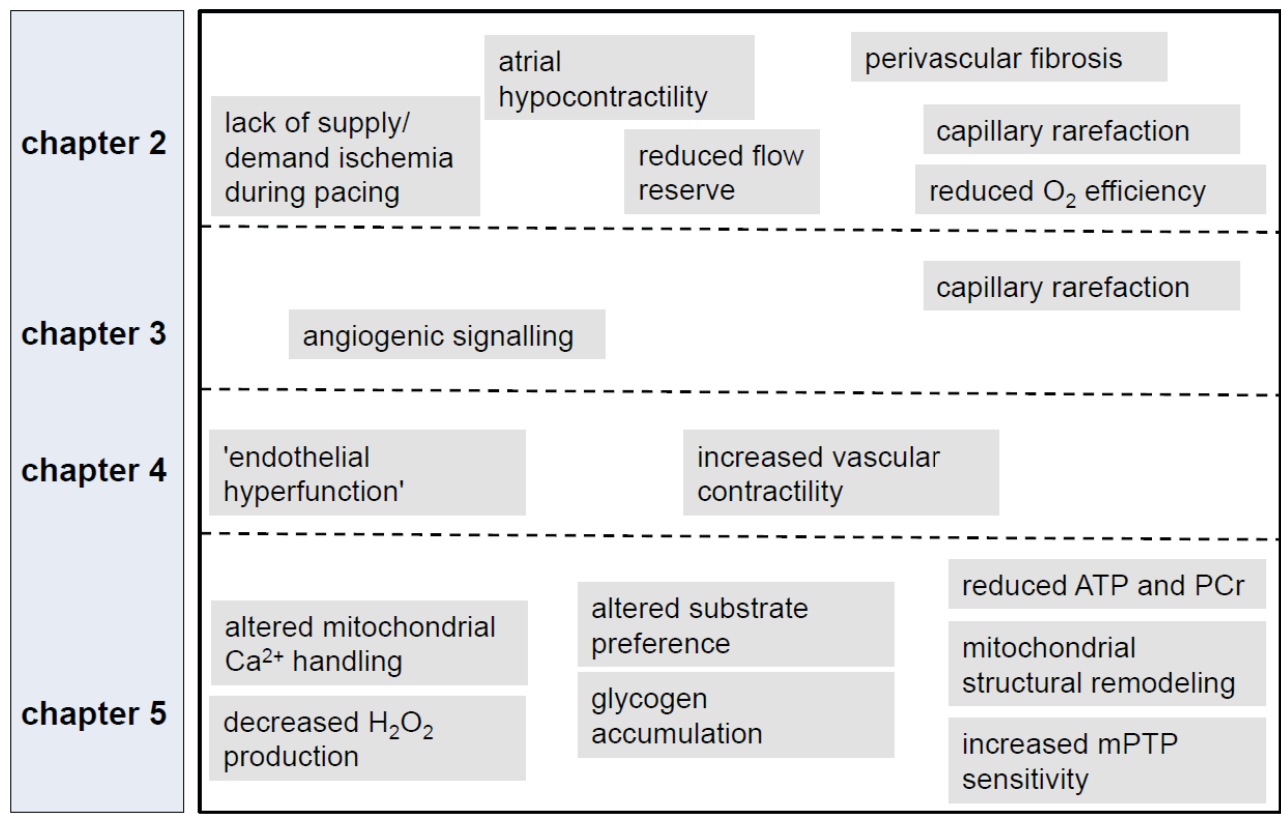

Figure 2. Metabolic and vascular remodeling: schematic representation of adaptive and maladaptive changes that are caused by AF (and CHF).

thesis represent some of the first reports on these forms of remodeling in models of AF. At this point, we can conclusively confirm that these phenomena do exist. We have only obtained limited information at time points that are relatively early in the pathogenesis of AF. Further research should include a more complete time course of these changes, at both earlier and much later time points.

Although we do believe that the phase we have studied is important in determining further degenerative changes, we cannot prove that metabolic and vascular remodeling play a causative role. Proving causation will require experimental interventions that either exacerbate or ameliorate various aspects of the mitochondrial and vascular changes that occur during AF. At this point, we can make a tentative scheme of the adaptive and maladaptive aspects of the remodeling processes we have described (figure 2). 'Adaptive' in this case would translate to 'increasing the capacity of the atria to cope with the stress that results from AF (or CHF)', while maladaptive changes would decrease this capacity. In some cases, we simply don't know whether a change has a positive or a negative impact on the atrial myocardium, e.g. in the case of glycogen accumulation. In other cases, a particular phenomenon may have both positive and negative consequences. For example, the atrial contractile dysfunction described in chapter 2 may be detrimental to cardiac 
function in failing hearts but may also prevent damage to the atrial myocardium by obviating supply/ demand ischemia. However, several aspects of metabolic and vascular remodeling, at the right side of the table, are likely to reduce the capacity of the atria to cope with stress represented by AF. As such, these factors form viable candidates for a causative role in progression of AF. 


\section{REFERENCES}

1. Wijffels MC, Kirchhof CJ, Dorland R, Allessie MA. Atrial fibrillation begets atrial fibrillation. A study in awake chronically instrumented goats. Circulation 1995;92:1954-1968.

2. Schotten U, Verheule S, Kirchhof P, Goette A. Pathophysiological mechanisms of atrial fibrillation: a translational appraisal. Physiological reviews 2011;91:265-325.

3. Spach MS. Anisotropy of cardiac tissue: a major determinant of conduction? Journal of cardiovascular electrophysiology 1999;10:887-890.

4. Verheule S, Tuyls E, Gharaviri A, Hulsmans S, van Hunnik A, Kuiper M, Serroyen J, Zeemering $S$, Kuijpers NH, Schotten U. Loss of continuity in the thin epicardial layer because of endomysial fibrosis increases the complexity of atrial fibrillatory conduction. Circulation Arrhythmia and electrophysiology 2013;6:202-211.

5. van Bragt KA, Nasrallah HM, Kuiper M, van Hunnik A, Kuijpers NH, Schotten U, Verheule S. Dynamic regulation of atrial coronary blood flow in healthy adult pigs. Heart rhythm : the official journal of the Heart Rhythm Society 2015;12:991-1000.

6. Duncker DJ, Bache RJ. Regulation of coronary blood flow during exercise. Physiological reviews 2008;88:1009-1086.

7. Boerth RC, Covell JW, Seagren SC, Pool PE. High-energy phosphate concentrations in dog myocardium during stress. The American journal of physiology 1969;216:1103-1106.

8. Britman NA, Levine HJ. Contractile Element Work: A Major Determinant of Myocardial Oxygen Consumption. The Journal of clinical investigation 1964;43:1397-1408.

9. Graham TP, Jr., Covell JW, Sonnenblick EH, Ross J, Jr., Braunwald E. Control of myocardial oxygen consumption: relative influence of contractile state and tension development. The Journal of clinical investigation 1968;47:375-385.

10. Bassenge E, Kucharczyk M, Holtz J, Stoian D. Treadmill exercise in dogs under -adrenergic blockade: adaptation of coronary and systemic hemodynamics. Pflugers Archiv : European journal of physiology 1972;332:40-55.

11. van Bragt KA, Nasrallah HM, Kuiper M, Luiken JJ, Schotten U, Verheule S. Atrial supplydemand balance in healthy adult pigs: coronary blood flow, oxygen extraction, and lactate production during acute atrial fibrillation. Cardiovascular research 2014;101:9-19.

12. Ausma J, Coumans WA, Duimel H, Van der Vusse GJ, Allessie MA, Borgers M. Atrial high energy phosphate content and mitochondrial enzyme activity during chronic atrial fibrillation. Cardiovascular research 2000;47:788-796.

13. Thijssen VL, Ausma J, Borgers M. Structural remodelling during chronic atrial fibrillation: act of programmed cell survival. Cardiovascular research 2001;52:14-24. 
14. Ausma J, Litjens N, Lenders MH, Duimel H, Mast F, Wouters L, Ramaekers F, Allessie M, Borgers M. Time course of atrial fibrillation-induced cellular structural remodeling in atria of the goat. Journal of molecular and cellular cardiology 2001;33:2083-2094.

15. Gramley F, Lorenzen J, Jedamzik B, Gatter K, Koellensperger E, Munzel T, Pezzella F. Atrial fibrillation is associated with cardiac hypoxia. Cardiovascular pathology : the official journal of the Society for Cardiovascular Pathology 2010;19:102-111.

16. Ogi H, Nakano Y, Niida S, Dote K, Hirai Y, Suenari K, Tonouchi Y, Oda N, Makita Y, Ueda S, Kajihara K, Imai K, Sueda T, Chayama K, Kihara Y. Is structural remodeling of fibrillated atria the consequence of tissue hypoxia? Circulation journal : official journal of the Japanese Circulation Society 2010;74:1815-1821.

17. Heil M, Eitenmuller I, Schmitz-Rixen T, Schaper W. Arteriogenesis versus angiogenesis: similarities and differences. Journal of cellular and molecular medicine 2006;10:45-55.

18. McGarry JD. Banting lecture 2001: dysregulation of fatty acid metabolism in the etiology of type 2 diabetes. Diabetes 2002;51:7-18.

19. Weaver ME, Pantely GA, Bristow JD, Ladley HD. A quantitative study of the anatomy and distribution of coronary arteries in swine in comparison with other animals and man. Cardiovascular research 1986;20:907-917.

20. Doenst T, Nguyen TD, Abel ED. Cardiac metabolism in heart failure: implications beyond ATP production. Circulation research 2013;113:709-724.

21. Gibbs CL. Cardiac energetics. Physiological reviews 1978;58:174-254.

22. Wisneski JA, Gertz EW, Neese RA, Gruenke LD, Craig JC. Dual carbon-labeled isotope experiments using D-[6-14C] glucose and L-[1,2,3-13C3] lactate: a new approach for investigating human myocardial metabolism during ischemia. Journal of the American College of Cardiology 1985;5:1138-1146.

23. Duncker DJ, Bache RJ, Merkus D. Regulation of coronary resistance vessel tone in response to exercise. Journal of molecular and cellular cardiology 2012;52:802-813.

24. Folkow B. Physiological aspects of primary hypertension. Physiological reviews 1982;62:347504 .

25. Egan B, Panis R, Hinderliter A, Schork N, Julius S. Mechanism of increased alpha adrenergic vasoconstriction in human essential hypertension. The Journal of clinical investigation 1987;80:812-817.

26. Ungvari Z, Koller A. Endothelin and prostaglandin $H(2)$ /thromboxane $A(2)$ enhance myogenic constriction in hypertension by increasing $\mathrm{Ca}(2+)$ sensitivity of arteriolar smooth muscle. Hypertension 2000;36:856-861.

27. Guazzi M, Arena R. Endothelial dysfunction and pathophysiological correlates in atrial 
fibrillation. Heart 2009;95:102-106.

28. Yoshino S, Yoshikawa A, Hamasaki S, Ishida S, Oketani N, Saihara K, Okui H, Kuwahata S, Fujita S, Ichiki H, Ueya N, Iriki Y, Maenosono R, Miyata M, Tei C. Atrial fibrillation-induced endothelial dysfunction improves after restoration of sinus rhythm. International journal of cardiology 2013;168:1280-1285.

29. Hornig B, Drexler H. Endothelial function and bradykinin in humans. Drugs 1997;54 Suppl $5: 42-47$.

30. Thompson MA, Henderson KK, Woodman CR, Turk JR, Rush JW, Price E, Laughlin $\mathrm{MH}$. Exercise preserves endothelium-dependent relaxation in coronary arteries of hypercholesterolemic male pigs. Journal of applied physiology 2004;96:1114-1126.

31. Gschwend S, Buikema H, Henning RH, Pinto YM, de Zeeuw D, van Gilst WH. Endothelial dysfunction and infarct-size relate to impaired EDHF response in rat experimental chronic heart failure. European journal of heart failure 2003;5:147-154.

32. Hambrecht R, Adams V, Erbs S, Linke A, Krankel N, Shu Y, Baither Y, Gielen S, Thiele H, Gummert JF, Mohr FW, Schuler G. Regular physical activity improves endothelial function in patients with coronary artery disease by increasing phosphorylation of endothelial nitric oxide synthase. Circulation 2003;107:3152-3158.

33. Reilly SN, Jayaram R, Nahar K, Antoniades C, Verheule S, Channon KM, Alp NJ, Schotten U, Casadei $\mathrm{B}$. Atrial sources of reactive oxygen species vary with the duration and substrate of atrial fibrillation: implications for the antiarrhythmic effect of statins. Circulation 2011;124:1107-1117.

34. Semenza GL. Hydroxylation of HIF-1: oxygen sensing at the molecular level. Physiology 2004;19:176-182.

35. Nagy JA, Dvorak AM, Dvorak HF. VEGF-A(164/165) and PIGF: roles in angiogenesis and arteriogenesis. Trends in cardiovascular medicine 2003;13:169-175.

36. Khairoun M, van der Pol P, de Vries DK, Lievers E, Schlagwein N, de Boer HC, Bajema IM, Rotmans JI, van Zonneveld AJ, Rabelink TJ, van Kooten C, Reinders ME. Renal ischemiareperfusion induces a dysbalance of angiopoietins, accompanied by proliferation of pericytes and fibrosis. American journal of physiology Renal physiology 2013;305:F901-910.

37. Rosenberger C, Heyman SN, Rosen S, Shina A, Goldfarb M, Griethe W, Frei U, Reinke P, Bachmann S, Eckardt KU. Up-regulation of HIF in experimental acute renal failure: evidence for a protective transcriptional response to hypoxia. Kidney international 2005;67:531-542.

38. Takagi H, Koyama S, Seike H, Oh H, Otani A, Matsumura M, Honda Y. Potential role of the angiopoietin/tiez system in ischemia-induced retinal neovascularization. Investigative ophthalmology \& visual science 2003;44:393-402.

39. Maisel WH, Stevenson LW. Atrial fibrillation in heart failure: epidemiology, pathophysiology, 
and rationale for therapy. The American journal of cardiology 2003;91:2D-8D.

40. Li D, Fareh S, Leung TK, Nattel S. Promotion of atrial fibrillation by heart failure in dogs: atrial remodeling of a different sort. Circulation 1999;100:87-95.

41. Hanna N, Cardin S, Leung TK, Nattel S. Differences in atrial versus ventricular remodeling in dogs with ventricular tachypacing-induced congestive heart failure. Cardiovascular research 2004;63:236-244.

42. Burstein B, Nattel S. Atrial fibrosis: mechanisms and clinical relevance in atrial fibrillation. Journal of the American College of Cardiology 2008;51:802-809.

43. Benjamin EJ, Levy D, Vaziri SM, D’Agostino RB, Belanger AJ, Wolf PA. Independent risk factors for atrial fibrillation in a population-based cohort. The Framingham Heart Study. Jama 1994;271:840-844.

44. Eckstein J, Verheule S, de Groot NM, Allessie M, Schotten U. Mechanisms of perpetuation of atrial fibrillation in chronically dilated atria. Progress in biophysics and molecular biology 2008;97:435-451.

45. Neuberger HR, Schotten U, Verheule S, Eijsbouts S, Blaauw Y, van Hunnik A, Allessie M. Development of a substrate of atrial fibrillation during chronic atrioventricular block in the goat. Circulation 2005;111:30-37.

46. Tsagalou EP, Anastasiou-Nana M, Agapitos E, Gika A, Drakos SG, Terrovitis JV, Ntalianis A, Nanas JN. Depressed coronary flow reserve is associated with decreased myocardial capillary density in patients with heart failure due to idiopathic dilated cardiomyopathy. Journal of the American College of Cardiology 2008;52:1391-1398.

47. Jayaweera AR, Wei K, Coggins M, Bin JP, Goodman C, Kaul S. Role of capillaries in determining $\mathrm{CBF}$ reserve: new insights using myocardial contrast echocardiography. The American journal of physiology 1999;277:H2363-2372.

48. Dedkov El, Zheng W, Christensen LP, Weiss RM, Mahlberg-Gaudin F, Tomanek RJ. Preservation of coronary reserve by ivabradine-induced reduction in heart rate in infarcted rats is associated with decrease in perivascular collagen. American journal of physiology Heart and circulatory physiology 2007;293:H590-598.

49. Cha YM, Dzeja PP, Shen WK, Jahangir A, Hart CY, Terzic A, Redfield MM. Failing atrial myocardium: energetic deficits accompany structural remodeling and electrical instability. American journal of physiology Heart and circulatory physiology 2003;284:H1313-1320.

50. Ehrlich JR, Nattel S, Hohnloser SH. Atrial fibrillation and congestive heart failure: specific considerations at the intersection of two common and important cardiac disease sets. Journal of cardiovascular electrophysiology 2002;13:399-405.

51. Mandinov L, Eberli FR, Seiler C, Hess OM. Diastolic heart failure. Cardiovascular research 2000;45:813-825. 
52. Wright S, Sasson Z, Gray T, Chelvanathan A, Esfandiari S, Dimitry J, Armstrong S, Mak S, Goodman JM. Left atrial phasic function interacts to support left ventricular filling during exercise in healthy athletes. Journal of applied physiology 2015;119:328-333.

53. Phan TT, Abozguia K, Shivu GN, Ahmed I, Leyva F, Patel K, Frenneaux M. Increased atrial contribution to left ventricular filling compensates for impaired early filling during exercise in heart failure with preserved ejection fraction. Journal of cardiac failure 2009;15:890-897.

54. Jikuhara T, Sumimoto T, Tarumi N, Yuasa F, Hattori T, Sugiura T, Iwasaka T. Left atrial function as a reliable predictor of exercise capacity in patients with recent myocardial infarction. Chest 1997;111:922-928. 
APPENDIX
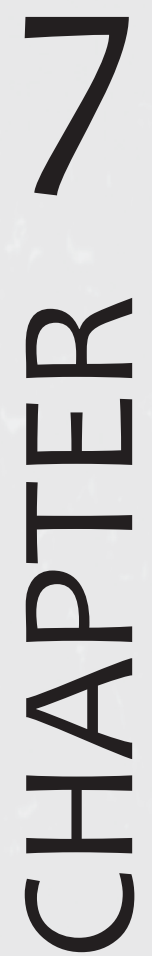


\section{SUMMARY}

The normal heartbeat originates in the sinus node. From there, the activation wave spreads over both upper chambers (atria), and also reaches the atrioventricular (AV) node, the only electrical connection between the upper chambers and the lower chambers (ventricles). Because conduction within the AV node is slow, there is a delay between atrial and ventricular activation. This delay allows the atria to empty themselves into the ventricles, before the ventricular contraction starts. During the ventricular contraction, blood is pumped by the right ventricle into the pulmonary artery and by the left ventricle into the aorta. There are numerous cardiac arrhythmias in which the activation sequence of the heart is disturbed. Of these arrhythmias, atrial fibrillation (AF) is the most common in clinical practice. In some patients, this arrhythmia occurs in the absence of other heart disease, so-called 'lone AF', but many patient have heart disease, for example heart failure' that predisposes to AF. During AF, activation waves in the atrium propagate rapidly (up to 600 times per minute) and irregularly, and organized atrial contraction is lost. Some of the atrial activations conduct through the AV node to the ventricles, leading to a fast (up to 180 beats per minute) and irregular ventricular rate. Patients with AF experience this rapid, irregular ventricular rate as palpitations, and their exercise tolerance is reduced because this rate cannot be properly regulated during physical exertion. In addition, on the long term AF patients have an increased tendency to develop blood clots, especially in the left atrium, leading to a higher risk of debilitating strokes and increased mortality.

In the beginning of the disease process, AF is often paroxysmal, meaning that AF occurs in episodes that terminate spontaneously. In many patients, the duration of these episodes gradually increase, until AF does not terminate spontaneously anymore (persistent AF). At this stage, sinus rhythm can often be restored by treatment with antiarrhythmic drugs. Eventually however, these drugs lose their efficacy and AF cannot be terminated by anti-arrhythmic drug treatment (permanent $A F)$. During the progression of the disease, AF itself leads to atrial 'remodeling'; changes in the atrial tissue that makes the arrhythmia more stable. Several key players in this process have already been identified and characterized extensively. A fast process of 'electrical remodeling' occurs rapidly, in the first hours to days of AF, and entails the shortening of the atrial action potential, allowing a higher activation frequency. A much slower process of 'structural remodeling', developing over months to years, changes the structure of the atrial tissue, increasing the size of atrial muscle cells (hypertrophy) and the amount of connective tissue (fibrosis) in between muscle cells. The latter in particular is thought to represent obstacles to conduction, increasing the complexity of fibrillatory conduction and therefore the stability of AF. Although these 'culprits' are well known, the treatment strategies to prevent the progression of AF are still unsatisfactory. One of the central problems is 
that we do not know exactly which factors cause electrical and structural remodeling. One of the candidate factors is the energy balance of the atrial myocardium. In a cardiac muscle cell (myocyte), the molecule adenosine triphosphate (ATP) delivers the energy that is required for contraction, electrical activity and other metabolic processes. An estimated $2 \%$ of the cellular ATP pool is utilized during each heartbeat. The rapid rates of electrical and contractile activity during AF will increase ATP consumption. This increased demand needs to be matched by increased ATP production, and the required oxygen and nutrients have to be supplied by the atrial vasculature. If the energy demand by the myocardium cannot be met, a state of supply/demand ischemia develops. The metabolic challenge presented by AF can be met by scaling back energy demand and by increasing energy supply, and there are several indications that both phenomena occur as a result of AF. Still, there is ample evidence that these adaptations fall short of redressing this disbalance, that may represent a driving force for atrial electrical as well as structural remodeling.

The general aim of this thesis is to investigate the metabolic and vascular consequences of AF itself, and of an important risk factor for AF, congestive heart failure (CHF). Characterizing and understanding the role of these processes may help in finding therapeutic approaches and targets that could inhibit the progression of AF.

As an animal model, we have chosen pigs, which are known to be similar to humans with respect to coronary anatomy, coronary blood flow regulation and metabolism. We studies animals early in the disease process, in the first few weeks, a phase in which we expected the metabolic and vascular derangement and adaptations to be most pronounced. First, we studied a pig model of CHF (chapter 2). Clinically, CHF is a major independent risk factor for $\mathrm{AF}$, with approximately $50 \%$ of patient with severe $\mathrm{CHF}$ developing AF. In the pig model, we induced $\mathrm{CHF}$ by rapid ventricular pacing. After 5 weeks, we observed that the degree to which both atrial and ventricular coronary vessels could dilate (i.e. increase blood flow to the cardiac muscle) was decreased. This in itself would reduce that capacity of the coronary vessels to accommodate fluctuation in energy and oxygen demand. However, during pacing (i.e. increased heart rate), we did not observe an increase in atrial lactate production, which would be a tell-tale sign of supply-demand ischemia. On the other hand, we demonstrate that atrial contractility has decreased dramatically in this model. Thus, the hypertrophied atria in this model still receive adequate coronary blood flow, but this does not translate to useful external work (contractility). In other words, the atrial oxygen efficiency (the amount of work delivered per amount of oxygen supplied) has decreased dramatically. In failing hearts, the atrial contraction becomes more important to overall cardiac function. The loss of the atrial contraction may contribute to reduced exercise capacity and exacerbation of heart failure, even before AF develops. 
Subsequently, we assessed metabolic and vascular alterations that occur because of AF itself. As a relatively early time point, we chose one weeks of AF (maintained by rapid atrial pacing). From these pigs, we isolated atrial small muscular arteries, the arteries that normally determine blood flow to the myocardium. Because virtually nothing was known about the behavior of these blood vessels and their reaction to AF, we performed a basic characterization of vascular function (chapter 4). We observed that both the reaction to vasodilators and vasoconstrictors was enhanced after one week of AF. This implies that coronary vessels, and thus coronary blood flow, react more strongly to factors that regulate vascular tone. To some extent, these alterations are similar to the (adaptive) changes that occur during exercise training. Further studies will have to determine whether these alterations in vascular function persist during the time course of AF, or whether vascular dysfunction eventually develops.

At the same point in time, we determined a number of metabolic parameters in tissue and assessed the function of isolated mitochondria. On the hand, we demonstrate a number of changes that can be interpreted as adaptations to increased energy demand, metabolic stress and calcium overload (increased mitochondrial respiratory control, decreased production of reactive oxygen species and faster calcium buffering). On the other hand, we observed potential counterproductive, maladaptive changes, including reduced tissue levels of energy carriers (ATP and $\mathrm{PCr}$ ), possibly reduced energy producing capacity (a reduction in the mitochondrial membrane constituent cardiolipins) and reduced mitochondrial calcium storage capacity during oxidation of fatty acids. The work we have performed in this study represents only a snapshot of metabolic changes during the pathogenesis of AF. While the time course of metabolic remodeling will have to be studied more closely, our observations do support the view that AF presents a major metabolic stress for atrial myocytes.

Finally, we have studied vascular structure at two time points, after 1 and 5 weeks of AF. First we show an increase at both time points of HIF1a expression, a classic marker of oxygen shortage. HIF1a activates the transcription of VEGF, one of the key factors in the formation of new blood vessels. Indeed, expression of VEGF was increased after 5 weeks of AF. Increased expression of VEGF would normally lead to the formation of new blood vessels to supply the tissue in which oxygen shortage occurs. However, when we determined the distribution of capillaries (the small vessels where oxygen supply to the tissue takes place), a paradoxical decrease in the number of vessels was apparent after 5 weeks of AF. This suggests that newly formed capillaries are not sufficiently stabilized and quickly disappear. At this point, we do not have a mechanistic explanation for the loss of capillaries from the atrial myocardium, despite hypoxic signaling. However, this phenomenon implies the capacity of the remodeled atria to respond to fluctuations in energy demand is 
limited and that it remains vulnerable to supply/ demand ischemia. 


\section{ARABIC SUMMARY}

الخلاصة

اضطر اب النظم القلبي أو اختلال النَّظم القَلِّْيّ هي الاختلافات التي تطر أ على دقات القلب و على نُظُم نقل الثارة الكهربائية في عضلة القلب. تختلف اضطر ابات النظم القلبي فيما بينها فمنها الأكثر خطورةً و الأقل خطورة على حياة المريض. إحدى حالات اضطراب النظم القلبي هو الرجفان الأذيني. يعتبر الرجفان الأذيني إحدى حالات اضطراب النظم القلبي الأكثر شيوعاً عند مرضى القلب، تكمن خطورة هذا المرض في كونه مسبب رئيسي في السكتة الدماغية. هنالك عدة عوامل تجعل الإنسان أكثر عرضة لمرض الرجفان الأذيني، على سبيل المثال تنشوهات الهيكل الأذيني الذي يعتبر السبب الرئيسي في حدوث الرجفان الأذيني. حتى الأن العوامل المسببة بحدوث تشوهات الهيكل الأذيني غير معروفة كلياً. لذلك كان الهدف العام من هذه الأطروحة هو دراسة وفهم الآليات والعوامل التي تشارك في حدوث تشوهات الهيكل الأذيني. فهم هذه الآليات يمكن أن يساعد في إيجاد النهج و الأهداف العلاجية التي من شأنها وقف مظاهر الرجفان الأذيني.إحدى أبرز الفرضيات لحدوث تشوهات الهيكل الأذيني هي نتيجة إختلال توازن الأوكسيجين في الأذين الأيسر.

لذلك لقد قمت في هذه الأطروحة بدراسة الأوعية الدموية وإعادة التمثيل الغذائي ولا سيما في الأذين الأيسر في ثلاثة نماذج من الخنازير.أولاً نموذج خنزير لديه قصور القلب الاحتقاني حيث تمت دراسة الأوعية الدموية وحركة إنقباض الأذين الأيسر ،ثانيأنموذج خنزير لديه رجفان أذيني قصير المدى حيث قمت هنا بدر اسة وظيفة و هيكل الثر ايين التاجية للاذين الأيسر كما قت أيضاً بدر اسة التمثيل الغذائي، هيكل، ووظيفة الميتوكوندريا.ثالثاً و أخيراً نموذج خنزير لديه رجفان أذيني طويل المدى حيث تم تقييم بنية الأو عية الدموية في الأذين الأيسر. تم العثورفي مجموعة قصور القلب الاحتقاني على انخفاض في حركة إنقباض الأذين، تضخم في خلايالأذين الأيسر ،انخفاض في احتياطي تدفق الدم في الثريان التاجي المؤدي إلى الأذين الأيسر وانخفاض في كثافة الأوعية الدموية في الأذين الأيسر. في مجموعة الرجفان الأذيني قصير المدى نم العثور على انخفاض في وظيفة التمثيل الغذائي ونقص الأوكسجين في الأذين الأيسر لكن هذا النقص لم يكن نتيجة تغير في وظيفة الثرايين التاجية للاذين الأيسر و إنما كان بسبب الزيادة في معدل حركة الأذين الأيسر خلال الرجفان الأذيني. 
وأخيراً في مجموعة الرجفان الأذيني طويل المدى وجدنا أيضاً نقص في الأوكسجين و انقباض في الأوعية الدموية للاذين الأيسر. فى الختام هذه الأطروحة تمكنت من ربط حالات الرجفان الأذيني بنقص الأوكسيجين للاذين الأيسر. كما تجدر الإشارة إلى أهية امدادات الاوكسجين للاذين الايسر وأن أي إعاقة لهذه الإمدادات يجعل الإنسان أكثر عرضة للرجفان الأذيني. كما أن الزيادة في سرعة الأذين التي تحدث نتيجة الرجفان الأذيني يؤدي إلى زيادة في الطلب على الاوكسجين مما يجعل الأذين أكثر عرضةً لحالات من نقص التروية التي تؤدي إلى إزدياد تشوهات الهيكل الأذيني. 


\section{OPPORTUNITIES FOR VALORIZATION}

\section{Relevance to healthcare}

Atrial fibrillation (AF) is the most common sustained tachyarrhythmia in clinical practice 1 . The prevalence of AF in the developed countries ranges between 1 to $2 \%$ of the general population ${ }^{2}$. In the Netherlands, an estimated 300,000 people have AF. The risk of developing AF strongly increases with age. In the Rotterdam study, performed on a large population of Dutch people aged 55 years and above, the overall AF prevalence was $5.5 \%$, with $0.7 \%$ prevalence in the $55-59$ years to $17.8 \%$ in those aged 85 years and above ${ }^{3}$. With the ageing of the general population in the western world, AF prevalence and the associated health care costs are expected to rise substantially over the next 50 years ${ }^{4}$.

Because AF leads to the formation of emboli, particularly in the left atrium, AF increases the risk of stroke: in 1 out of every 5 strokes AF is the underlying cause ${ }^{5}$. This is the most important reason that AF is also associated with increased mortality ${ }^{6,7}$. As a consequence, AF represents a large, and growing burden on our healthcare system 8,9 .

Current AF therapy is still unsatisfactory. In the patients in whom sinus rhythm can be restored by electrical of pharmacological cardioversion, AF very often recurs. The risk of stroke can be reduced by anti-coagulation therapy, but with potentially harmful side-effects such as uncontrolled bleeding, including cerebral hemorrhage. The efficacy of ablation therapy is limited in patients with (longstanding) persistent AF.

In many patients, AF gradually becomes harder to treat as a result of a slow process of structural remodeling. This process includes i.e. fibrosis, cellular hypertrophy and fatty infiltration ${ }^{10,11}$ that increase the complexity of fibrillatory conduction and thereby the stability of AF. Despite extensive research, the exact mechanisms responsible for structural remodeling are still poorly understood. Treatment options that inhibit structural remodeling could halt AF progression in patients.

\section{Novelty of the concept}

Because of their obvious relevance in diseases such as ischemic heart disease and heart failure, (left) ventricular metabolism and blood supply have been investigated in great detail for decades. By comparison, we know very little about atrial metabolism and tissue perfusion, how they are affected by risk factors for AF, and the way in which they contribute to the pathogenesis of AF.

In this thesis, we have characterized some aspects of atrial metabolic and vascular remodeling as a result of atrial fibrillation (AF) and congestive heart failure (CHF, a major risk factor for AF. Understanding the pathophysiological processes occurring in the atria would help in identifying new therapeutic targets/approaches that could 
improve the treatment AF.

$A F$ is characterized by fast and irregular atrial activation. The rapid rates of electrical activity and contraction during AF must present an enormous challenge to the energy balance of atrial myocytes. This challenge can be met by scaling back energy demand and by increasing energy supply, and there are several indications in the literature that both phenomena occur as a result of AF. Still, there is also ample evidence that these adaptations fall short of redressing this disbalance, that may represent a driving force for atrial electrical as well as structural remodeling.

\section{Road to product}

The data in this thesis strongly support the existence of metabolic and vascular remodeling. We have observed striking changes in vascular regulation and mitochondrial physiology after one week of AF. Despite the activation of angiogenic signalling, capillary rarefaction had occurred after 5 weeks of AF. In the model of $\mathrm{CHF}$, vascular remodeling coincided with profound atrial contractile dysfunction that is likely to accelerate the development of heart failure.

We show that AF and CHF have a substantial impact on the atrial metabolism and vasculature, but our knowledge on these effects is still quite limited. On the whole, our data are consistent with supply/ demand ischemia and consequent metabolic and vascular remodeling in the important early phase of AF progression. However, our present data represent only snapshots of the pathogenesis of AF, and other time-points, both earlier and later, will have to be studied in a similar way to fully characterize these processes. This should include characterization of metabolic and vascular remodeling in a model of paroxysmal AF, where each AF episode may represent a renewed ischemic insult to the atrial myocardium, leading to an accumulation of (structural) damage.

At present, evidence that metabolic and vascular remodeling play a causative role in electrical and structural remodeling are largely circumstantial. Evidence for causation would be strengthened using large animal models of AF by testing intervention strategies that selectively target determinants of the atrial supply/ demand balance. A possible approach is stimulation of atrial angiogenesis and/ or stabilization of newly formed capillaries to increase atrial perfusion in fibrillating atria. Alternatively, a treatment strategy could be targetted at 'improving' atrial metabolism ${ }^{12}$ to enhance oxygen efficiency and preserve atrial function. If successful in large animal models, such interventions could lead to new treatment options in AF patients. This would eventually lead to highly desirable 'upstream therapy' to prevent the progression of AF in patients. 


\section{REFERENCES}

1. Wyndham CR. Atrial fibrillation: the most common arrhythmia. Tex Heart Inst J 2000;27:257267.

2. Camm AJ, Lip GY, De Caterina R, Savelieva I, Atar D, Hohnloser SH, Hindricks G, Kirchhof P, Guidelines ESCCFP. 2012 focused update of the ESC Guidelines for the management of atrial fibrillation: an update of the 2010 ESC Guidelines for the management of atrial fibrillation. Developed with the special contribution of the European Heart Rhythm Association. Eur Heart J 2012;33:2719-2747.

3. Heeringa J, van der Kuip DA, Hofman A, Kors JA, van Herpen G, Stricker BH, Stijnen T, Lip $\mathrm{GY}$, Witteman JC. Prevalence, incidence and lifetime risk of atrial fibrillation: the Rotterdam study. Eur Heart J 2006;27:949-953.

4. European Heart Rhythm A, European Association for Cardio-Thoracic S, Camm AJ, Kirchhof P, Lip GY, Schotten U, Savelieva I, Ernst S, Van Gelder IC, Al-Attar N, Hindricks G, Prendergast B, Heidbuchel H, Alfieri O, Angelini A, Atar D, Colonna P, De Caterina R, De Sutter J, Goette A, Gorenek B, Heldal M, Hohloser SH, Kolh P, Le Heuzey JY, Ponikowski $\mathrm{P}$, Rutten FH, Guidelines ESCCFP. Guidelines for the management of atrial fibrillation: the Task Force for the Management of Atrial Fibrillation of the European Society of Cardiology (ESC). Europace 2010;12:1360-1420.

5. Risk factors for stroke and efficacy of antithrombotic therapy in atrial fibrillation. Analysis of pooled data from five randomized controlled trials. Arch Intern Med 1994;154:1449-1457.

6. Krahn AD, Manfreda J, Tate RB, Mathewson FA, Cuddy TE. The natural history of atrial fibrillation: incidence, risk factors, and prognosis in the Manitoba Follow-Up Study. Am J Med 1995;98:476-484.

7. Go AS, Hylek EM, Phillips KA, Chang Y, Henault LE, Selby JV, Singer DE. Prevalence of diagnosed atrial fibrillation in adults: national implications for rhythm management and stroke prevention: the AnTicoagulation and Risk Factors in Atrial Fibrillation (ATRIA) Study. JAMA 2001;285:2370-2375.

8. Ball J, Carrington MJ, McMurray JJ, Stewart S. Atrial fibrillation: profile and burden of an evolving epidemic in the 21st century. Int J Cardiol 2013;167:1807-1824.

9. Sheikh A, Patel NJ, Nalluri N, Agnihotri K, Spagnola J, Patel A, Asti D, Kanotra R, Khan H, Savani C, Arora S, Patel N, Thakkar B, Patel N, Pau D, Badheka AO, Deshmukh A, Kowalski $\mathrm{M}$, Viles-Gonzalez J, Paydak H. Trends in Hospitalization for Atrial Fibrillation: Epidemiology, Cost, and Implications for the Future. Prog Cardiovasc Dis 2015;58:105-116.

10. Li D, Fareh S, Leung TK, Nattel S. Promotion of atrial fibrillation by heart failure in dogs: atrial remodeling of a different sort. Circulation 1999;100:87-95.

11. Allessie M, Ausma J, Schotten U. Electrical, contractile and structural remodeling during 
atrial fibrillation. Cardiovasc Res 2002;54:230-246.

12. Horowitz JD, Chirkov YY, Kennedy JA, Sverdlov AL. Modulation of myocardial metabolism: an emerging therapeutic principle. Curr Opin Cardiol 2010;25:329-334. 


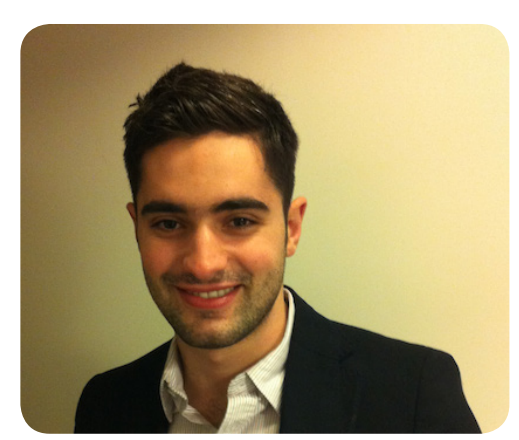

Hussein M. Nasrallah

\section{ABOUT THE AUTHOR}

15.11.1985

2003

2004-2008

2008-2010

01.01.2011 till 01.08.2011

2011-2015

\section{Awards}

2014

2008-2009
Born in Beirut/Lebanon

Life Sciences (High school diploma)

BSc. Biomedical Engineering in Beirut, Lebanon

MSc. Biomedical Engineering in Aachen, Germany

Research assistant, Extracorporeal circulation Lab Aachen, Germany

PhD candidate department of physiology cardiovascular research Institute Maastricht, The Netherlands

Best Poster award in the Dutch endothelial biology society (Biezenmortel)

RWTH Aachen scholarship for International students 


\section{LIST OF PUBLICATIONS}

1. van Bragt KA, Nasrallah HM, Kuiper M, van Hunnik A, Kuijpers NHL, Schotten $U$, Verheule S. Dynamic regulation of atrial coronary blood flow in healthy adult pigs. Heart Rhythm. 2015;12:991-1000

2. van Bragt KA, Nasrallah HM, Kuiper M, Luiken JJ, Schotten U, Verheule S. Atrial supply-demand balance in healthy adult pigs: coronary blood flow, oxygen extraction, and lactate production during acute atrial fibrillation. Cardiovascular Research. 2014;101:9-19

\section{Submitted to Circulation Heart Failure}

Nasrallah HM, van Bragt KA, Kuiper M, van Hunnik A, Schotten U, Verheule S. Left atrial oxygen supply in a pig model of congestive heart failure: vascular remodeling atrial contractile dysfunction and reduced oxygen efficiency

\section{Submitted to Cardiovascular Research}

Pasdois P*, Nasrallah HM*, Kuiper M, Beauvoit B, Luiken JJ, Loyer V, Dos Santos $P$, Lendeckel $U$, Schild L, Jaïs $P$, Schotten U, Verheule S. Early metabolic and mitochondrial remodelling in a pig model of atrial fibrillation

\section{*Both Authors contributed equally to the work}

\section{Submitted to Cardiovascular Research}

Opacic D, van Bragt K, Nasrallah HM, Schotten U, Verheule S.

Atrial metabolism and tissue perfusion as determinants of electrical and structural remodeling in atrial fibrillation 


\section{ACKNOWLEDGMENT}

If I look into my project, my findings and the tasks that I have accomplished in the last four years I just could not believe that all this work would be possible without the significant help and collaboration from so many people who supported me to fully accomplish the goals of this study. Hereby, I would like to offer my gratitude and appreciation to each and everyone who helped, supported and believed in me also to those who stood beside me in critical moments of my life.

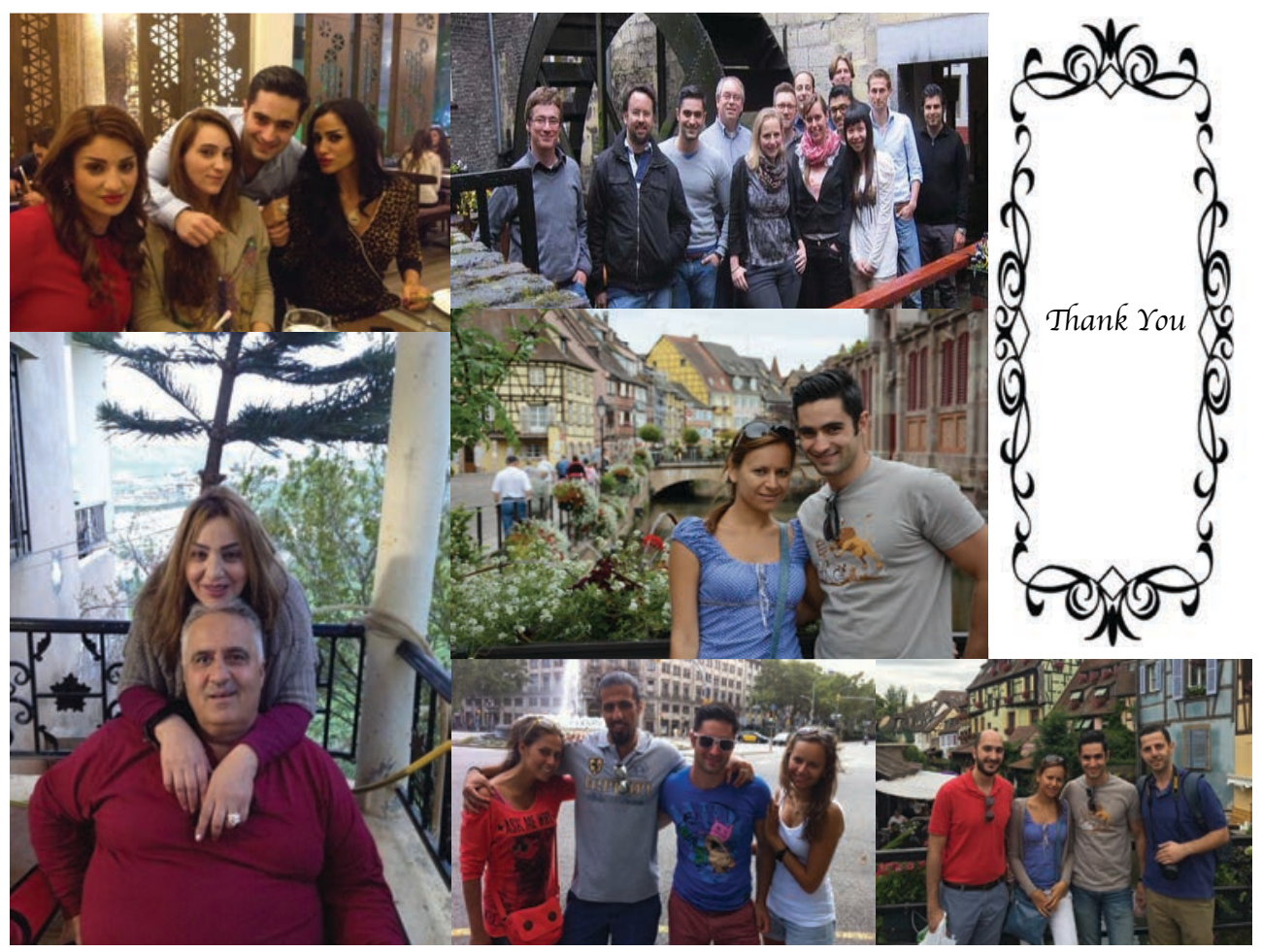

First, my deepest appreciation goes to my supervisor professor Ulrich Schotten. Uli, I would like to thank you first for believing in me and second for giving me the opportunity to become a $\mathrm{PhD}$ candidate at your lab. I still remember all the beneficial comments you gave me and trust me they will always be in my mind. Uli, I feel very proud to be your PhD student because I know that you are a successful man and a real scientist.

The second and most important gratitude goes to my supervisor Dr. Sander Verheule. Sander, it was a great pleasure for me to be your PhD student. It was also a great opportunity for me to listen to your thoughts, your ideas and to learn from you how does a brilliant scientist thinks. It is always amazing for me how much you have knowledge about various aspects: religion, politics, music, sports and science. I always enjoyed listening to all your ideas and your great sense of humor. Regarding 
my PhD project, your office was always opened for me to listen to my remarks, questions and your suggestions were always correct; I never remember that you gave any remark related to my project which wasn't right. Sander for all your help and support allow me once again to thank you for all what you have delivered to me and I really think that without you my project would not be successful.

I would also like to sincerely thank my thesis committee members: Prof. Harry Crijns, Prof. Mark Post, Prof. Dirk Duncker and Prof. Ed van Bavel to accept reviewing my thesis. A special thank goes to Paul Schiffers for all his contribution and assistance in introducing me into the topic of vascular pharmacology and training me to be able to isolate and mount vessels in an arteriograph and a myograph. Thanks a lot Paul for giving me the opportunity to work together, which eventually resulted in performing one of the major chapters within my thesis. I would like to thank Joost Luiken and Will Coumans for the great collaboration and the assistance they offered. Furthermore, I would like to thank all my colleagues at the department of physiology. Marion it was a great pleasure for me to work with you all the assistance and hard work you invested into helping me with my project is very much appreciated. I really think that you are a valuable person in the department of physiology. Kelly, thank you very much for all the assistance you provided me while starting up with my project I also enjoyed your spontaneous sense of humor, which always made me laugh. Arne, I was always fascinated with the surgical skills you acquire. It was great opportunity to work with you. I always appreciated your effort in helping me I also would like to thank you for all the assistance and the feedback you provided into my project. Dragan, thank you for the help you have provided me, I always enjoyed chatting with you about so many aspects related to science, work and others. Dragan, I am very much confident that you will manage you $\mathrm{PhD}$ in a perfect way. I also wish your big dream of becoming a vascular surgeon will become truth. To my friend Ajay a lot of gratitude and appreciation for all his support during my PhD phase and I also wish him very good luck In his PhD. Stijn, my dear friend I think we really became very good friends the last four years, I would like to thank you because I know that you believed in me and you trusted me; our chats in the room and our funny jokes will always be remembered. Stephanie, my friend it was a great that you became a member in our lab; I enjoyed chatting with you about life, work and sport. It was a great idea from you to do the insanity workout. I really learned a lot from you how to be positive and to enjoy life. Additionally, thank you for all the help you provided me while editing parts of my book also listening to my presentations and giving me your great feedback. Ali my great friend thank you for the support you provided me. It was always great to go together for a smoke and chat about work, politics and share our jokes and frustrations. I will never forget our trip to the USA (Boston and New york) particularly our adventures in the Bronx.

Pawel and BG you were great members of the lab I learned a lot from you guys. It 
was always a pleasure to listen to your intellectual thoughts and if I can say wisdom about various aspects of culture, politics and religion. Piotrick and Patrick thank you so much for being my colleagues and friends I enjoyed going out with you few times for a beer and burger. It was also nice to talk to you guys. Bianca and Vivian thank you so much for all the great assistance you provided me during the last four years. Whenever I required your assistance you were always friendly and supportive. Members of the animal facility Sanne, Petra, Huup and Joyce thank you so much for all the assistance and help you delivered in helping me and especially looking into the animals mainly making sure that the animal welfare is considered carefully. $A$ special thank goes to my very good friend Ali and his great wife Dayana for all their support and the nice moments we spent the last years. Another word of gratitude goes to my great friends Bilal, Houssein, Abbas and Mohammad; thank you guys a lot for believing in me and being always supportive. To my amazing sisters Chirine, Sahar and Samah you are the most valuable things in my life. Thank you very much for your help and support. You always stood beside me, believed and trusted me. I really love you and there are no words that can express my appreciation to you.

Reaching the final parts of my acknowledgment word. I would thank my Mother and Father for all the great help they provided me standing beside me in every moment of my life and especially during the tough moments. Mom and Dad from the start of my path you have always believed in me, you trusted that I would definitely be able to finish my PhD from the start. You have always been great support for me I don't forget your words of hope and wisdom to continue and accomplish what I have dreamed about. I love you guys "Allah yakhleekon foq rasna amen ya rab". Final thank in my acknowledgment goes to my great partner Lenka. Trust me Lenka there are no words that could express my gratitude to you. Since the beginning of my PhD you believed in me, you where always next to me from the start you have never gave up on me considering all the tough moments of stress and frustrations. I always found you next to me backing me up and believing in me. Finally, one sentence from the heart "it is always great and amazing for me to be with you". 